\begin{abstract}
Title of thesis:

BELIEFS AND ATTITUDES REGARDING HPV VACCINATION AMONG COLLEGE-AGE WOMEN: AN APPLICATION OF THE HEALTH BELIEF MODEL
\end{abstract}

Kathryn L. Schaefer, Master of Arts, 2010

$\begin{array}{ll}\text { Thesis directed by: } & \text { Mary Ann Hoffman, Ph.D } \\ & \text { Professor } \\ & \text { Department of Counseling and Personnel Services }\end{array}$

The human papillomavirus (HPV) represents the most common sexually transmitted disease, however the development of the HPV vaccine, Gardasil, protects women from becoming infected. The current study examined attitudes toward the HPV vaccine in 150 college-age women who had received the vaccine and 58 college-age women who had not. Data were collected using an online survey and correlations and regression analyses were run to assess for relationships between the variables of interest. Using the Health Belief Model (HBM), predictor variables included perceived benefits, barriers, susceptibility and severity regarding HPV infection and vaccination. Additional psychosocial variables were also explored. Results indicate that for unvaccinated women, perceived benefits accounted for unique variance in predicting vaccine intentions. Moreover, self-efficacy, cues to action and subjective norms all accounted for unique variance in differentiating vaccinated from unvaccinated women. In summary, women's decision to get the vaccine involves a complex interplay of factors. 


\title{
BELIEFS AND ATTITUDES REGARDING HPV VACCINATION AMONG COLLEGE-AGE WOMEN: AN APPLICATION OF THE HEALTH BELIEF MODEL
}

\author{
By \\ Kathryn L. Schaefer \\ Thesis submitted to the Faculty of the Graduate School of the \\ University of Maryland, College Park, in partial fulfillment \\ of the requirements for the degree of \\ Master of Arts \\ 2010
}

Advisory Committee:

Professor Mary Ann Hoffman, Chair

Professor Clara Hill

Associate Professor Robin Sawyer 
(C) Copyright by Kathryn Schaefer 2010 


\section{Table of Contents}

Table of Contents

List of Tables iv.

List of Figures $\quad$ v.

Chapter 1: Introduction 1

Chapter 2: Review of the Literature $\quad 9$

Human Papillomavirus $\quad 11$

Prevalence and Detection 11

Physical and Psychological Consequences $\quad 12$

Risk Factors $\quad 16$

HPV Vaccine 19

Health Belief Model 23

Model Structure $\quad 23$

Meta-Analyses $\quad 26$

HPV Vaccine Acceptance $\quad 30$

HPV Vaccination and College-Age Women 32

Subjective Norms $\quad 38$

HPV Knowledge $\quad 39$

Sexual Risk Factors $\quad 43$

Chapter 3: Statement of the Problem 46

HPV Vaccination Intentions $\quad 50$

Research Hypotheses $\quad 50$

HPV Vaccination Status $\quad 52$

Research Hypotheses $\quad 52$

Research Questions $\quad 56$

$\begin{array}{ll}\text { Chapter 4: Method } & 60\end{array}$

$\begin{array}{ll}\text { Design } & 60\end{array}$

Participants 61

Measures $\quad 63$

$\begin{array}{ll}\text { Procedure } & 72\end{array}$

$\begin{array}{ll}\text { Chapter 5: Results } & 75\end{array}$

Preliminary Analyses $\quad 75$

Sample Description of Demographics and Sexual Risk Factors 76

$\begin{array}{ll}\text { Preliminary Correlations } & 88\end{array}$

Exploratory Factor Analysis for the HBM Variables 93

Analysis of Hypotheses and Research Questions 105

HPV Vaccination Intentions 105 
Research Hypotheses 105

HPV Vaccination Status 107

Research Hypotheses 107

Research Questions 111

Analysis of Open-Ended Questions 113

Chapter 6: Discussion $\quad 120$

Overview of the Sample 120

Findings about Unvaccinated Women 122

Findings Comparing Vaccinated and Unvaccinated Women 125

Findings about Vaccinated Women 130

Open-Ended Questions 132

Overall Summary of Findings $\quad 141$

Limitations 145

Implications for Research and Practice 147

Appendices

Appendix A: Demographics 155

Appendix B: Sexual Risk Factors 157

Appendix C: HBM Questionnaire 159

Appendix D: Self-Efficacy 163

Appendix E: Cues to Action 164

Appendix F: Subjective Norms 165

Appendix G: HPV Knowledge 166

Appendix H: Decision-Making 168

Appendix I: HPV Vaccination Status 169

Appendix J: HPV Vaccination Intentions $\quad 170$

Appendix K: Informed Consent 171

Appendix L: Participant Remuneration $\quad 172$

Appendix M: Links to HPV Information 173

Appendix N: Email to Participants 174

Appendix O: EFA for Susceptibility 176

Appendix P: EFA for Severity 177

Appendix Q: EFA for Benefits 178

Appendix R: EFA for Barriers 179

$\begin{array}{ll}\text { References } & 180\end{array}$ 


\section{List of Tables}

Table 1: Demographic characteristics of participants

Table 2: Sexual risk factors

Table 3. Means, standard deviations, and internal consistencies for HBM measure

Table 4. Means, standard deviations, and internal consistencies for other measures

Table 5. Structure coefficients for perceived susceptibility

Table 6. Structure coefficients for perceived severity

Table 7. Structure coefficients for perceived benefits

Table 8. Structure coefficients for perceived benefits revised 1

Table 9. Structure coefficients for perceived benefits revised 2

Table 10. Structure coefficients for perceived barriers

Table 11. Structure coefficients for perceived barriers revised

Table 12. Structure coefficients for the HBM: benefits, barriers, susceptibility and severity

Table 13. Summary of hierarchical regression predicting vaccine intentions

Table 14. Summary of hierarchical logistic regression predicting vaccine status

Table 15. Responses to the greatest benefits of HPV vaccination

Table 16. Responses to the greatest drawbacks of HPV vaccination

Table 17. Responses to the factors that led to HPV vaccination

Table 18. Responses to the factors that keep participants from getting the HPV vaccine 


\section{List of Figures}

Figure 1. Correlations for vaccinated women

Figure 2. Correlations for unvaccinated women

Figure 3. Correlations for total sample 


\section{Chapter One}

Introduction

In recent years, prevention research has gained a stronger presence among counseling psychologists (Romano \& Netland, 2008). The demands of the $21^{\text {st }}$ century, such as cost containment and a focus on prevention rather than remediation, as well as individual and institutional needs have fueled the growth of prevention science. Critical issues that impact the health of individuals and communities require a preventative perspective, as opposed to remediating the problems after they develop. Counseling psychology represents an ideal field to promote this perspective since it has roots in prevention, education, and the promotion of human development across the lifespan.

Part of prevention research involves exploring which factors influence individual's health decisions and behaviors. Research indicates that strong and consistent individual differences exist in health behaviors (Armitage \& Conner, 2000). While some of these individual differences are attributable to sociodemographic variables, other differences have been found to relate to social cognitive variables such as perceived benefits and barriers to taking action. Researchers have generally focused on social cognitive variables to explain differences in health behavior since they are potentially more amenable to change. The present study explored how social cognitive factors within the Health Belief Model influenced an individual's decision to receive a vaccine that prevents infection of the human papillomavirus (HPV), a widespread sexually transmitted disease with potentially serious consequences.

The Health Belief Model (HBM) represents a useful framework for understanding how individuals form health decisions that subsequently influence health behavior. 
Originally designed to explain participation in disease prevention programs, this theory has also been used to explain the change and maintenance of health behavior as well as guide health behavior interventions (Strecher \& Rosenstock, 1997). For instance, the HBM has been used to predict heart disease preventive behaviors, dietary compliance, and vaccine acceptance (Ali, 2002; Becker, Maiman, Kirscht, Haefner, \& Drachman, 1977; Chapman \& Coups, 1999). As a value-expectancy theory, the HBM contains two health representations, threat perception which involves susceptibility and severity of an illness and behavioral evaluation which involves benefits and barriers of taking action (Sheeran \& Abrahan, 1996). Therefore, individuals are expected to take action to prevent illness if they perceive themselves as susceptible to a condition that has potentially serious consequences and believe that a course of action would be beneficial as well as outweigh any barriers (Strecher, Champion, \& Rosenstock, 1997).

While the four main components of the HBM provide a valuable foundation for understanding health behavior, other social cognitive variables have been added by some researchers to augment the model. One variable that has been used on occasion in health studies includes cues to action. These cues include a range of internal and external triggers that can promote or discourage health protective behaviors (Sheeran \& Abrahan, 1996). For example, in one study, physician advice or recommendation represented a successful cue to action that influenced individuals to receive the flu vaccine (Cummings et al., 1979). When combined with the appropriate beliefs, cues to action can promote health behavior (Sheeran \& Abrahan, 1996). Thus, if the perceived threat of disease is high and the perceived benefits outweigh perceived barriers, then a positive cue to action can prompt behavior to avoid the illness (Strecher et al., 1997). 
Self-efficacy has also been added to the Health Belief Model in many studies to increase the theory's explanatory power (Rosenstock, Strecher, \& Becker, 1988). Bandura (1977, p.79) described self-efficacy as "the conviction that one can successfully execute the behavior required to produce the outcomes". In the case of health behavior, the desired outcome is generally preventing an illness or living a healthier lifestyle. Research supports the importance of self-efficacy in predicting initiation and maintenance of behavioral change, especially for more complex and long-term changes (Bandura, 1986; Strecher, DeVellis, Becker, \& Rosenstock, 1986; Strecher et al., 1997). Self-efficacy has been used to explain health behaviors such as eating, drinking, exercising, smoking, and sexual practices (Strecher et al., 1997). Therefore, the HBM combined with self-efficacy theorizes that successful behavior change depends upon a high perception of threat, belief that change will be beneficial and come at an acceptable cost, and confidence in the ability to overcome perceived barriers to taking action.

The social cognitive variables previously described focus solely on the individual, however, most people do not make health decisions in isolation. One factor that considers the influence of others on health behavior is subjective norms. This construct assesses whether an individual perceives that his or her social network expects and approves of the behavior and whether the individual's social contacts perform the behavior themselves (Fishbein \& Azjen, 1975). Traditionally associated with the Theory of Planned Behavior, subjective norms have been found to influence protective sexual intentions and behavior among college students. Higher subjective norms positively influenced condom use, getting tested for HIV, and receiving the HPV vaccine (Munoz-Silva, Sanchez-Garcia, Nunes, \& Martins, 2007; King, 2006, Boehner et al., 2003; Jones \& Cook, 2008; Costar, 
2008). Thus, the behavior of peers and the approval of significant others appear to play an important role in health decisions and behaviors.

While social cognitive variables are more amenable to change, certain sociodemographic variables can still provide important information regarding health decisions. For instance, knowledge about a disease or behavioral risk factors for a disease can influence whether an individual engages in behaviors to protect against the disease. Some studies indicate that higher human papillomavirus (HPV) knowledge and certain sexual risk factors for HPV increase intentions to receive a vaccine that protects against HPV (Jones \& Cook, 2008; Allen Mohllajee, Shelton, Othus, Fontenot, \& Hanna, 2009; Gerend \& Magloire, 2008; Jones \& Cook, 2008; Boehner, Howe, Bernstein, \& Rosenthal, 2003; Costar, 2008; Buchanan, 2008). Moreover, knowledge and behavioral risk factors are more changeable than other sociodemographic characteristics and can be modified through interventions. For instance, HPV educational interventions have been found to increase knowledge about the human papillomavirus (Lambert, 2001; Dempsey, Zimet, Davis, \& Koutsky, 2003; Chan, Cheung, Lo, \& Chung, 2007). Therefore, these variables were included as factors that could influence health decisions about receiving the HPV vaccine.

The human papillomavirus (HPV) represents an important disease to study since it affects a large majority of the population. As many as $75 \%$ of sexually active men and women will become infected with HPV in their lifetime (Cates, 1999; Koutsky, 1997). Young adults between the ages of 15 and 25 are particularly affected since peak prevalence and the highest rate of new infections occur in this age group (NIH, 1996; Weinstock, Berman \& Cates, 2004). Although HPV is often harmless and asymptomatic, 
it can cause bothersome consequences in both men and women such as genital warts as well as deadly diseases such as oral, cervical and anogenital cancers (Graziottin \& Serafini, 2009). Detecting HPV is problematic since many people are unaware that they are infected. Moreover, the virus passes through genital skin-to-skin contact, making it easily transmissible between sexual partners (Cates et al., 1999). Although awareness of HPV has grown in recent years, misunderstandings about this sexually transmitted disease still persist (Lambert, 2001).

In the past, HPV prevention efforts focused on promoting Pap smear screenings, condom use and abstinence, none of which fully protect against HPV. While Pap smear tests can detect cervical HPV infection in women, not all sexually active females receive regular screenings (Fletcher \& Bryden, 2005). Moreover, Pap smears do not prevent HPV and once the virus is acquired, treatments to remove precancerous lesions can be painful and may result in complications (American Society for Colposcopy and Cervical Pathology, 2006). Condoms also do not fully protect against HPV since the virus is transmitted via skin-to-skin contact and areas not covered by the condom are vulnerable to infection (Winer et al., 2006). Finally, abstinence might not be a realistic option for some young adults and, although rare, virgins can still acquire HPV (Winer et al., 2003). Given the limitations of HPV prevention efforts, a new, more effective method is necessary in order to fully prevent HPV infection.

In 2006, an HPV vaccine, Gardasil, was developed to protect females against four different strains of HPV. Two of the strains are associated with $90 \%$ of genital warts and two of the strains cause $70 \%$ of cervical cancers (Center for Disease Control and Prevention, 2008). In a randomized double-blind placebo-controlled trial, the HPV 
vaccine reduced the incidence of the four HPV types by $90 \%$ (Villa et al., 2005).

Therefore, the vaccine protects against infection of the four HPV types as well as the clinical sequelae of genital warts and cervical cancer. Prior research indicates that the majority of college-aged women are interested in the HPV vaccine and 85 to 88 percent of participants intend to become vaccinated (Gerend \& Magloire, 2008; Jones \& Cook, 2008; Kahn, Rosenthal, Hamann \& Bernstein, 2003). While some studies have explored how individual differences among women might affect their decision to receive the HPV vaccine, only one known study has focused on women who have already received the HPV vaccine. Including vaccinated women increases the understanding of which factors positively influence vaccination and which factors serve as barriers.

Allen et al. (2009) conducted a study in 2007 illustrating the psychosocial factors associated with HPV vaccine adoption among college women ages 18 to 22 . The study used the original Health Belief Model variables of perceived susceptibility, perceived severity, perceived benefits and perceived barriers as well as the additional variables of HPV knowledge, subjective norms and demographic characteristics including sexual activity. The outcome variable included stages of HPV vaccine adoption such as deciding not to receive the vaccine, being unaware of the vaccine, planning to receive the vaccine, starting the vaccine injections, and being fully vaccinated. Results of the study present evidence that the HBM as well as additional social cognitive factors significantly predict stages of vaccine adoption. These findings are useful in understanding how individual differences influence various stages of health decision-making and preventative behavior related to the HPV vaccine. The present study partially replicated and extended these research findings using the same health topic of vaccination against HPV. 
The present study had three purposes: 1) To explore sociodemographic and social cognitive variables among non-HPV vaccinated college-age women, 2) To explore sociodemographic and social cognitive variables among HPV vaccinated college-age women, and 3) To compare vaccinated and unvaccinated college-age women along sociodemographic and social cognitive dimensions. The first purpose of the study was accomplished by looking at predictors of unvaccinated college-age women's intentions to receive the vaccine. The second purpose of the study was a relatively unexplored area that helped illuminate the reasons behind getting vaccinated. Finally, the third purpose of the study was important since the opportunity for HPV vaccination was available for several years suggesting that women who had not yet received the vaccine might be different from women who had received the vaccine.

As with Allen et al.'s (2009) study, the Health Belief Model served as the social cognitive framework and other psychosocial variables, including subjective norms, HPV knowledge and HPV sexual risk factors, augmented the model. However, the present study made several important additions to the existing literature. Cues to action and selfefficacy were included as psychosocial predictors of HPV vaccination behavior and intentions. These factors have often been added to the HBM to increase the explanatory power of the model and helped provide new insights into beliefs about the HPV vaccine. In addition, this study presented information about how women view the HPV vaccine after it has been available for three years. Most previous research, including Allen et al. (2009), took place prior to or directly after the availability of the vaccine when women may have known little about HPV vaccination. Women's beliefs about the vaccine during the early phases of dissemination might be different from current views now that the 
vaccine has been established. Finally, college-aged women were an important population to research since they were eligible for vaccination and possessed some of the highest rates of HPV. As few studies have examined variables related to HPV vaccination and intentions in women ages 18 to 26 , the present study provided a useful contribution to the literature. 


\section{Chapter Two}

Review of the Literature

Human papillomavirus (HPV) represents a widespread sexually transmitted disease that can cause serious consequences for both women and men. These consequences include genital warts and cancers of the cervix, vulva, vagina, penis, anus and the oropharynx area of the mouth and throat. Although both men and women are affected by HPV, the focus of this study was on college-aged women who are particularly susceptible since peak HPV prevalence and a high rate of new infections occur in this age group (Cervical Cancer, 1996; Bauer et al., 1991; Weinstock et al., 2004). The development of the HPV vaccine Gardasil, however, protects women from becoming infected and from infecting their sexual partners. This vaccine represents an opportunity to eliminate health concerns caused by HPV which would have enormous public health benefits. However, since the HPV vaccine is not currently mandatory, the success of the vaccine depends upon an individual's decision to become vaccinated. Understanding how women view the vaccine represents an important step in reducing the prevalence of HPV.

The HPV vaccine is recommended to be given to 11 and 12-year old girls before they engage in any skin-to-skin sexual contact, therefore most HPV studies have focused on parents' attitudes and beliefs for vaccinating their daughters. However, women up to age 26 are eligible for a "catch-up" vaccine and can still benefit from vaccination. The vaccine protects women from four different strains of HPV, therefore, even if a female is infected with one strain of HPV, the vaccine can still protect her from three other strains (Adams et al., 2009; Jones \& Cook, 2008). Vaccinating college-aged women can greatly reduce the incidence of HPV infection for the woman getting the vaccine and can reduce 
spreading the virus to partners. Few studies have explored college-age women's beliefs regarding the HPV vaccine. Since college women are at the age where they, not their parents, will make the decision to become vaccinated, it is important to understand the factors that influence HPV vaccination in this population. The Health Belief Model (HBM) was used as the conceptual framework for this study and was augmented by additional constructs including cues to action, self-efficacy, subjective norms, HPV knowledge and sexual risk factors.

This literature review will begin by reviewing the clinical relevance of HPV for the general population and for college-age women specifically. A description of the virus's prevalence and detection, physical and psychological consequences, and risk factors will be provided. The literature review will then focus on the development of the HPV vaccine and its acceptance among college-age women. Finally, factors that predict HPV vaccination will be explored, including the main components of the Health Belief Model (HBM) as well as additional factors such as cues to action, self-efficacy, subjective norms, HPV knowledge and sexual risk factors. Specifically, the review will discuss the model structure of the HBM, describe several meta-analyses and explain how the HBM has been used to predict HPV vaccine acceptance in general and among college-age women. Factors outside of this theoretical framework, such as subjective norms, HPV knowledge and sexual risk factors, and their relevance to HPV vaccination in college-age women will also be explored. 


\section{Human Papillomavirus}

\section{Prevalence and Detection}

Approximately 20 million people in the United States are infected with HPV, the most common sexually transmitted infection (Center for Disease Control and Prevention, 2008). Research indicates that up to $75 \%$ of sexually active adults will become infected in their lifetime (Cates, 1999; Koutsky, 1997). Peak prevalence occurs in young adults between the ages of 22 and 25 and one study discovered HPV infection in $46 \%$ of college females (NIH, 1996; Bauer et al., 1991). Moreover, 74\% of new HPV infections occur among 15-24 year-olds (Weinstock, Berman \& Cates, 2004).

HPVs are a small group of DNA viruses. Over 100 types of HPV exist and approximately one-third infect the genital tract (Graziottin \& Serafini, 2009). The HPV types that infect the genital tract fall into either high risk or low risk categories. High risk types of HPV can cause anogenital and oral cancers, whereas low risk types of HPV can cause genital warts. Most HPV infections are cleared by the immune system or become latent (Graziottin \& Serafini, 2009; Sellors et al., 2003). However, without a Pap smear it is impossible to know whether HPV is still present in the cervix (Costar, 2008). Pap smears test for unusual cell changes in the cervix caused by HPV. There is no FDAapproved test to detect HPV in men or to determine a person's overall HPV status for either men or women. The only way to detect HPV in men is through a biopsy of an HPV-related tumor in the oral pharyngeal or anogenital areas. HPV cannot be seen and most people do not develop symptoms, such as genital warts or cancerous lesions. Moreover, HPV can stay dormant in the body for years after acquisition of the virus. 
Therefore, many people are not aware that they are infected and can easily transmit the virus to a partner.

Unlike other sexually transmitted diseases that spread through fluid transmission, HPV is transmitted through genital skin-to-skin contact (Cates et al., 1999). Recent research indicates that consistent condom use among newly sexually active women can reduce the risk of HPV infection (Winer et al., 2006). However, condoms cannot fully protect against the virus since HPV can infect areas not covered by the condom. The only way to completely prevent HPV is to abstain from any sexual contact. Individuals in a monogamous relationship can also lower their risk of HPV infection if their partner has had no or few other sexual partners.

Physical and Psychological Consequences

HPV can have serious consequences for both women and men. Studies have found that HPV is the primary cause of cervical cancer and can also cause cancers of the anus, vulva, vagina, penis, and mouth (National Cancer Institute, 2009; Souza et al., 2007). However, HPV is particularly associated with negative consequences for women's health. Specifically, HPV types 16 and 18 are associated with approximately $70 \%$ of cervical cancers (Clifford, Smith, Plummer, Munoz \& Franceschi, 2003; Munoz et al., 2003; Bosch \& Sanjose, 2003). Cervical cancer is the second most common cancer in women worldwide and a common cause of death in middle aged women from developing countries (Bosch \& Sanjose, 2003). Although the availability of screening options has greatly reduced the incidence of invasive cervical cancer in developed countries, precursors to cervical cancer, such as lesions, remain a public health burden. HPV has 
been detected in $95 \%$ to $100 \%$ of cervical cancer cases, supporting the claim that HPV is the first necessary cause of a human cancer ever identified (Walboomers et al., 1999). Much research and publicity has focused on HPV's association with cervical cancer, however HPV is also associated with substantial proportion of anogenital and oral cancers. In fact, the number of HPV-related noncervical cancers diagnosed annually in the US approximately equals the number of cervical cancers (Gillison, Chaturvedi, \& Lowy, 2008). HPV 16 accounts for the largest proportion of HPV-related oral, anal, penile, vulvar, and vaginal cancers. Specifically, HPV 16 was detected in $84 \%$ of anal cancer specimen, $84.2 \%$ of penile cancer specimen, $29.3 \%$ of vulvar cancer cases and 55.4\% of vaginal cancer cases (Frisch, 1997; Pascual, 2007; Smith, Backes, Hoots, Kurman, \& Pimenta, 2009). In addition, HPV 16 infection precedes oral cancer development and is the necessary cause of as many as $72 \%$ of oral pharyngeal cancer cases (Souza et al., 2007; Mork et al., 2001). While the incidence of cervical cancer has been decreasing, the incidence of oral and anal cancers has been increasing in the US since the 1960's and 70's (Gillison, 2008; Chaturvedi, Engels, Anderson, \& Gillison, 2008; Melbye, Rabkin, Frisch, \& Biggar, 1994). Anal cancer is usually underestimated in women, but is now higher in women than in men (Melbye, et al., 1994; Graziottin \& Serafini, 2009). On the other hand, HPV-related oral cancer is higher in men than in women. Anal and oral cancers remain particularly serious since there are currently no effective or widely used screening procedures for these diseases (Gillison et al., 2008). Although the risks of particular types of HPV-related cancer may be more prevalent in one gender over the other, transmission of the virus by infected men and women can still cause cancer in their partner. Therefore, the HPV vaccine is one of the few current ways 
to manage infection in both the women receiving the vaccine and in the women's partners.

Low risk HPV viruses can cause genital warts which, although benign, still cause significant physical and psychological distress (Graziottin \& Serafini, 2009). Specifically, HPV types 6 and 11 are associated with nearly 90\% of genital warts (Gissman et al., 1983). Prevalence studies indicate that the incidence of genital warts has increased since the 1950s (Becker, Stone, \& Alexander, 1987; Chuang, Perry, Kurland, Ilstrup, 1984). Currently in the United States, $5.6 \%$ of adults report ever having been diagnosed with genital warts, with higher percentages in women than in men (Dinh, Sternberg, Dunne, \& Markowitz, 2008). Specifically, women between the ages of 25 and 34 years have the highest percentage. However, since this data relies on self-report rather than actual detection, the prevalence of genital warts might actually be higher. Genital warts are highly contagious and develop in about two-thirds of people who have sexual contact with someone who has the condition (Mayo Clinic, 2009). The infection can even be transmitted from mother to baby during pregnancy and can cause physical complications when giving birth (Mayo Clinic, 2009).

Aside from causing physical problems such as pain and discomfort, genital warts can also cause psychological distress (Conaglen, Hughs, Conaglen, \& Morgan, 2001; Maw, Reitano, \& Roy, 1998; Ireland, Reid, Powell, \& Petrie, 2005). Feelings of disgust, shame, and embarrassment are common (Maw et a., 1998). People with genital warts have a significant lower health-related quality of life due to pain/discomfort and anxiety/depression as compared to those without the condition (Woodhall et al., 2008). Moreover, the psychological impact of a diagnosis of genital warts may be greater for 
women than for men. For instance, women's perception of their health was worse than men's after a genital warts diagnosis (Woodhall et al., 2008). Aside from the psychological consequences, genital warts also present a large economic burden costing more than $\$ 700$ per 1000 person-years of enrollment (Insinga, Dasbach, \& Myers, 2003). This economic burden is greatest among females aged 15-24 years.

HPV infection can lead to serious consequences, not just physically but psychologically as well. Following diagnosis and treatment of HPV, women reported a decreased interest in sex, a worsening in the emotional relationship with their partner, and feelings of anxiety and fear of cervical cancer (Maggino et al., 2007; Filiberti et al., 1993; Linnehan \& Groce, 1999). In addition, women who tested positive for HPV experienced significantly more anxiety and distress, and felt worse about past, current, and future sexual relationships as compared to HPV-negative women (McCaffrey et al., 2004). Moreover, college-based health providers who have diagnosed and treated HPV infections generally perceive HPV as having a significant psychosocial impact on college women (Linnehan \& Groce, 1999). They agreed that HPV infection can cause feelings of anger, guilt, blame, and fear as well as raise concerns about self-image, sexuality, and sexual functioning among college women. The majority of providers also reported that addressing emotional and psychosocial issues was the most challenging aspect of managing HPV. Therefore, HPV appears have a substantial effect on women's emotional, social, and sexual functioning.

HPV and its clinical sequelae also present a significant economic burden. Treatment for genital warts and precancerous cervical lesions that result from HPV involve removing problematic cells, decreasing HPV viral load and watching for 
recurrence (Graziottin \& Serafini, 2009). These procedures are costly and consume significant health care resources. In fact, the annual health care costs of HPV-related conditions in the United States range from 2.25-4.6 billion dollars, making the burden of HPV second only to HIV for sexually transmitted diseases (Fleurence, Dixon, Milanova, $\&$ Beusterien, 2007). This is not surprising given that there are an estimated 6.2 million new cases of high risk HPV infection in the US each year (Trottier \& Franco, 2006; Center for Disease Control and Prevention, 2008). In women under age 24 alone, the estimated total annual cost of HPV is 2.8 billion dollars (Chesson, Blandford, Gift, Tao, \& Irwin, 2004). Therefore, HPV infection in young women comprises the majority of annual health care costs for HPV.

\section{Risk Factors}

Since HPV is primarily a sexually transmitted disease, risk for acquiring the infection is heavily influenced by sexual activity (Koutsky, 1997). Women who have a higher number of lifetime sexual partners and who engaged in intercourse at an early age are at higher risk for HPV (Kataja, et al., 1993; Pichichero, 2007; Winer et al., 2003). Specifically, women with five or more partners are more likely to become infected than women with one or no sexual partners (Kataja et al., 1993). Studies indicate that college women have an average number of between 1.8 and 5.6 sexual partners, however this information could be underreported (Winer et al., 2003; Gerend \& Magliore, 2008; Reinisch, Sanders, Hill, Ziemba-Davis, 1992). A woman's risk is also dependent on certain characteristics of her sexual partner. For instance, the likelihood of acquiring HPV increases if the male partner is uncircumcised, has an STD, or has had a higher number of sexual partners (Kataja, et al., 1993; Castellsague et al., 2002; Burk et al., 1996). 
However, HPV infection is not strictly limited to those engaging in intercourse. Although rare, virgins can also become infected since any type of non-penetrative sexual contact can transmit the virus. One study found a prevalence rate of $7.9 \%$ in virginal women over the course of two years (Winer et al., 2003). Non-penetrative sexual contact was associated with increased risk of HPV infection among virgins, but not among sexually active women.

Besides the risk factors already listed, studies focusing specifically on college females found additional characteristics associated with HPV. Ho, Bierman, Beardsley, Chang, and Burk (1998) discovered that Hispanic and African-American women were at increased risk for HPV as compared to Caucasian women. Although the age range was narrow, younger women had a higher likelihood of acquiring HPV than older women. Moreover, increased risk was associated with high frequencies of vaginal sex and alcohol consumption, anal sex, and a partner not currently in school. Some of these factors, such as anal sex and alcohol consumption might serve as a proxy for other risky sexual behavior. Finally, Winer et al. (2003) found that the presence of a new male sexual partner within the last eight months led to a higher likelihood of HPV infection. Given the high rate of sexual activity and alcohol consumption on college campuses, a large majority of college women are at high risk for acquiring HPV. Studies of sexually active college females indicate an average number of 1.8 to 5.6 sexual partners and high rates of unprotected sexual activity, as well as a lack of preventive measures such as getting a Pap smear (Reinisch et al., 1992; Vail-Smith \& White, 1992; Burkett et al., 1992; Linnehan \& Groce, 1999). Moreover, while a woman may have one or few partners, her risk is still based on the number of sexual partners that her partner(s) has had as well. The sexual 
behaviors of college women and their partners can increase their risk of acquiring HPV and explain the high incidence of infection among this age group.

Some studies have also found an association between HPV infection and nonsexual behaviors, such as oral contraceptive use and smoking (Kataja, et al., 1993; Winer et al., 2003). However, the literature remains inconclusive since other studies have not supported these findings. For instance, Mosciki et al. (2001) found that use of oral contraceptives actually had a significant protective effect against HPV. Since oral contraceptives have been associated with cervical cancer, it has been hypothesized that use of oral contraceptives alter the progression of HPV rather than increase the risk of acquiring the infection (Koutsky, 1997; WHO, 1993). While the majority of previous research has failed to find an association between smoking and HPV, some evidence suggests that smoking is associated with cervical cancer (Mosciki et al., 2001; Barton et al., 1988). Therefore, it is possible that smoking could act in a similar manner as oral contraceptives and change the progression of the infection in individuals who have HPV. However, these studies are still in the preliminary stages and require additional research to ascertain the true nature of the association.

HPV and its clinical sequelae present a large economic, physical and psychological burden. This highlights the importance of developing methods to reduce the incidence of HPV and thereby diminish the consequences of the virus. Current methods of HPV prevention, such as condom use and abstinence, present incomplete protection as well as other major limitations. As indicated previously, condom use does not completely protect against HPV since the virus gets transmitted through skin-to-skin contact. Moreover, abstinence presents an unrealistic expectation for many college 
women and, although rare, virgins can still acquire HPV. Current screening procedures, such as Pap smears, help detect the presence of HPV and reduce the risk of cervical cancer. However, these procedures do not prevent HPV infection or the development of precancerous lesions, which can result in psychological distress and unpleasant surgical procedures. Moreover, cervical screenings are not effective for women who fail to receive regular Pap smears. Finally, as indicated previously, widely used and effective screening procedures do not exist for other HPV-related cancers, such as anal and oral cancers. Due to these limitations, a vaccine that prevents the acquisition of HPV presents a more effective strategy for reducing HPV-related diseases in both men and women.

\section{HPV Vaccine}

In 2006, the U.S. Food and Drug Administration approved Merck's Gardasil, an HPV vaccine that protects females against HPV 6 and 11 which cause $90 \%$ of genital warts and HPV 16 and 18 which cause 70\% of cervical cancers (Center for Disease Control and Prevention, 2008). HPV 16 is also associated with oral, anal, penile, vulvar, and vaginal cancers. In a randomized double-blind placebo-controlled trial, the HPV vaccine reduced the incidence of HPV 6, 11, 16 and 18 by 90\% (Villa et al., 2005). Moreover the vaccine reduced the incidence of clinical disease, including genital warts, vulvar, vaginal, and cervical precancerous cellular changes, and cancers associated with these types of HPV by 100\% (Garland et al., 2007). In a separate double-blind placebocontrolled trial, the HPV vaccine efficacy for preventing cervical lesions was 98\% (Future II Study Group, 2007). Therefore, the HPV vaccine not only reduced the incidence of HPV, but also substantially reduced the incidence of HPV-related diseases and precancerous lesions. 
Gardasil is administered via three injections costing about $\$ 125$ each over six months and is currently licensed for females ages 9 to 26 years (Center for Disease Control and Prevention, 2008; Gardasil, 2009). While the vaccine is most effective for girls and women not yet infected with the vaccine-type HPV strains, women previously infected with the virus are no less immune to developing other related or unrelated types of HPV (Thomas et al., 2000). Therefore, even women previously exposed to HPV types $6,11,16$ or 18 may benefit from additional protection as a consequence of a vaccineinduced antibody response (Adams, Jasani, \& Fiander, 2009). The vaccine, however, does not treat existing HPV infections. Gardasil has recently been licensed for males and preliminary clinical trials suggest that an HPV 16/18 vaccine was effective and well tolerated in boys aged 10 to 18 years (Petaja et al., 2009). Research on vaccine strategies indicate that even after vaccinating 12-year-old boys and girls, a catch-up vaccine for women ages 12-24 can substantially reduce the disease in the short term (Elbasha, Dasbach, \& Insinga, 2007). In fact, a vaccination strategy for 12-year-old boys and girls that did not include a catch-up program was less effective and more costly. Given that the peak prevalence of HPV infection occurs in women ages 22 through 25, vaccination in college-aged women is particularly important (NIH, 1996; Burak \& Meyer, 1997).

As of May 1, 2009, at least 24 million doses of Gardasil have been distributed in the United States (CDC, 2009). Since this time, there have been reports of adverse events from the vaccine, $93 \%$ of which have been considered non-serious. The most common reported side effects have included fainting, pain and swelling at the injection site, headache, nausea, and fever. Fainting after vaccines and injections is especially common in adolescents. While serious adverse events have also been reported, causal associations 
cannot be made between these events and the vaccine. The reports merely mean that the event occurred sometime following vaccination. Overall, there have been 26 confirmed reports of death, several cases of a rare disorder, Guillain-Barré Syndrome, that causes muscle weakness, and few reports of blood clots among females who have received the vaccine (CDC, 2009). However, medical experts have not found any common medical patterns that would suggest that these events were caused by the vaccine. Moreover, the percentage of serious adverse events reported for Gardasil has been less than the overall average for other vaccines (CDC, 2008). Still, these reports have caused concerns among consumers and anti-vaccine groups about the safety of the vaccine (Houppert, 2007).

However, the main controversy surrounding the HPV vaccine has focused on whether states should make the vaccine mandatory for preteen girls. Some people disapprove of a school mandate to vaccinate due to concerns over the drug's cost, safety, and parents' rights to refuse (National Conference of State Legislatures (NCSL), 2009). Since HPV is a sexually-contracted virus, Christian conservative groups who favor an abstinence-only approach have argued that Gardasil will increase promiscuity in young women (Houppert, 2007). Moreover, states must address funding issues if the vaccine becomes mandatory, including whether to require coverage by insurance plans. These debates are still on-going for the majority of state legislatures and will likely continue for the next several years.

Despite the concerns and controversies surrounding Gardasil, college women tend to hold positive attitudes overall toward the HPV vaccine. The majority of college women are interested in Gardasil and 85 to 88 percent of participants intend to become vaccinated (Gerend \& Magloire, 2008; Jones \& Cook, 2008; Kahn, Rosenthal, Hamann 
\& Bernstein, 2003). However, studies conducted in 2007 indicate that only $12 \%$ of college-aged females have received at least one vaccine injection (Allen et al., 2009; Grant, Kravitz-Wirtz, Breen, Tiro \& Tsui, 2009). While low vaccination rates can be expected during the first year after vaccine licensing, this rate is still well below the number of adolescents who received the vaccine. In 2009, the Center for Disease Control (CDC) conducted a national survey of vaccination coverage of adolescents and found that approximately $37 \%$ of 13 to 17 -year old females had initiated the HPV vaccine series (CDC, 2009). However, only $18 \%$ received the three shots needed for full protection. Although the vaccine is administered over the course of six months, this time frame does not seem to fully account for the discrepancy between those who initiate the vaccine series and those who complete it. A national survey has not been conducted among college-aged women, therefore, it remains difficult to paint an accurate picture of HPV vaccine uptake among this population. Understanding the beliefs and attitudes regarding the HPV vaccine among college-age women represents an important step in creating a successful HPV vaccination program. College students will likely decide for themselves whether to receive the HPV vaccine, and their beliefs and attitudes can influence their acceptance. Determining which factors positively influence vaccination and which factors serve as barriers can help create health messages and other interventions to promote health protective decision-making. One theory that is often used in understanding healthprotective decision-making is the Health Belief Model (HBM). This theory includes components relevant to preventative behaviors such as receiving the HPV vaccine. 


\section{Health Belief Model}

\section{Model Structure}

The HBM represents one of the most widely used conceptual frameworks in health behavior (Strecher \& Rosenstock, 1997). As such, this model served as the framework for this study and was augmented with additional variables, including cues to action and self-efficacy. Developed by a group of social psychologists in the 1950s, HBM was originally used to explain the wide-spread failure of people to participate in programs to prevent disease. Since this time, the model has been used to explain the change and maintenance of health behavior and has served as a guiding framework for health behavior interventions. The HBM is a value-expectancy theory where value refers to the desire to avoid illness or to get well and expectancy refers to the belief that a specific health action would prevent illness (Strecher \& Rosenstock, 1997). Two health representations, threat perception and behavioral evaluation, emerged as the central aspects of the model (Sheeran \& Abrahan, 1996). Threat perception depends upon beliefs regarding the perceived susceptibility to illness and the anticipated severity of the illness. Behavioral evaluation encompasses two beliefs as well, those regarding the benefits of a recommended health behavior and those regarding the barriers or costs to enacting the behavior. Therefore, individuals are expected to take action to prevent illness if they perceive themselves as susceptible to the condition, believe that the condition has potentially serious consequences, believe that an available course of action would be beneficial and anticipate that the barriers to taking action would be outweighed by the benefits (Strecher, Champion, \& Rosenstock, 1997). 
Perceived susceptibility, perceived severity, benefits and barriers comprise the four main components of the HBM. While the definition of these constructs has varied across research, Strecher et al. (1997) offers general descriptions of the model components. Specifically, perceived susceptibility refers to an individual's subjective assessment of his or her risk for contracting an illness. Perceived severity, on the other hand, refers to an individual's feelings about the seriousness of contracting an illness. This includes both medical aspects such as pain, and psychosocial consequences such as interference with social roles. Benefits refer to an individual's beliefs regarding the effectiveness of a particular health action in reducing the disease threat. This can also involve both medical and psychosocial aspects. Finally, barriers refer to the potential negative aspects of a particular health action, and can involve both practical and psychological costs. Practical barriers could include aspects such as time, expense, availability and waiting time. Psychological barriers could include costs such as pain, embarrassment and threat to well-being.

Aside from the four main components of the model, other constructs have been used to augment the model in order to further explain a person's health behavior. People's health decisions generally involve a complex interplay of factors that might not be completely captured by a rational, cost-benefit model like the HBM. Cues to action were included in various early formulations of the HBM, but have not been systematically studied or included in the majority of health belief research (Strecher et al., 1997). These cues can represent internal triggers, such as perceptions of symptoms, as well as external triggers like social influence and health education campaigns. Since the HPV vaccine has received significant media attention, including advertisements, 
incorporating cues to actions in the model seems particularly relevant for this study. When combined with the appropriate beliefs, cues to action can promote health behavior (Sheeran \& Abrahan, 1996). If the perceived threat of disease is high and the perceived benefits outweigh perceived barriers, then a cue to action can prompt behavior to avoid the illness (Strecher et al., 1997). For instance, one study discovered that physician advice or recommendations represented successful cues to action for flu vaccination (Cummings et al., 1979). However, cues to action can also discourage health behavior if an individual is reminded of potential costs or barriers associated with the action. In another flu vaccination study, knowing someone who experienced negative side effects from the vaccine negatively impacted inoculation behavior (Aho et al., 1979). The fleeting nature of cues to action makes this concept difficult to study, particularly in explanatory surveys (Strecher et al., 1997). In addition, Weinstein (1988) maintains that cues to action do not represent rational, expectancy-value components like the rest of the HBM. Furthermore, little research exists on the types of cues that are related to taking action or the number or frequency of cues. Therefore, cues to action represent an important component that has been largely neglected in research on the HBM.

In the 1970s, Bandura introduced the concept of self-efficacy, or the belief in one's ability to successfully perform the behavior required to produce certain outcomes (Strecher et al., 1997). Rosenstock, Strecher, and Becker (1988) proposed adding selfefficacy to the Health Belief Model in order to increase its explanatory power. They hypothesized that this new model would more fully account for health-related behavior as compared to the earlier model, and would lead to more effective behavioral interventions. Research suggests that self-efficacy accounts for a significant amount of initiation and 
maintenance of behavioral change, especially for more complex and long-term changes (Strecher et al., 1997). Simple preventative actions that are easy to carry out, such as a one-shot immunization, might not depend as much on self-efficacy. However, selfefficacy would likely be an important determinant for receiving the HPV vaccination which requires three shots over the course of six months. In addition, receiving the HPV vaccine requires that women acknowledge that they are sexually active or plan to become sexually active. This acknowledgement requires self-efficacy beyond simply arranging to receive the vaccine. Therefore, successful behavior change depends upon a high perception of threat, belief that change will be beneficial and come at an acceptable cost, and confidence in the ability to overcome perceived barriers to taking action.

Meta-Analyses

Several meta-analyses have been conducted on studies utilizing the HBM. Janz and Becker (1984) reviewed 46 studies published between 1974 and 1984, and calculated significance ratios for susceptibility, severity, benefits, and barriers. The studies in the review focused on a variety of health behaviors such as influenza vaccination, breast selfexaminations and participation in genetic screening programs. The significance ratios calculated by Janz and Becker (1984) indicate the percentage of times each construct was statistically significant across all of the studies. Overall, susceptibility was significant in $81 \%$ of the studies, severity in $65 \%$, benefits in $78 \%$, and barriers in $89 \%$. Therefore, the significance ratios support the importance of the HBM in predicting health behaviors with barriers as the most reliable predictor. In addition, the findings from prospective studies suggest that these health beliefs play a causal role. The meta-analysis results also indicate that the relative importance of the HBM's components may vary based on the 
health behavior. For instance, severity had only a moderate effect upon preventive behavior and clinic utilization, but it was the second most powerful predictor of the behavior of individuals recently diagnosed as ill (Janz \& Becker, 1984; Sheeran \& Abraham, 1996). Thus, severity might be more difficult to conceptualize when asymptomatic or when the health threat only occurs in the long term. This applies to the HPV vaccine since vaccination represents a preventative behavior and the consequences of HPV, such as cervical cancer, may occur long after infection. Therefore, severity might not represent a strong predictor of HPV vaccination behavior.

While Janz and Becker's (1984) meta-analysis provides strong support for the HBM across a variety of health behaviors, the study contains several limitations. First, the significance ratios only indicate the frequency of significant HBM components rather than the size of their effect (Sheeran \& Abraham, 1996). Moreover, these ratios give equal weight to all studies regardless of sample size which could bias the results. Finally, not all of the studies in the meta-analysis included all four main components of the HBM which could influence the significance of each individual predictor. These issues were addressed in a subsequent meta-analysis by Harrison, Mullen, and Green (1992).

Harrison et al. (1992) identified 234 published empirical studies of the HBM, however, only 16 measured all four main components and included reliability checks. This illustrates the large number of studies that fail to operationalize the full HBM model and provide psychometric tests of the measures. Pearson's $r$ effect sizes were calculated across the 16 studies and transformed into a weighted average for each component. Health behavior had an average 0.15 correlation with susceptibility, 0.08 with severity, 0.13 with benefits, and -0.21 with barriers. While these correlations were statistically 
significant, they represent small effect sizes and account for just between 1.5 and $4 \%$ of the variance in behavior. Moreover, the effect sizes displayed considerable variability across studies, indicating that study design, measurement, or conceptualization differences likely influenced the results. The researchers also found a difference in the importance of HBM components between retrospective and prospective studies. Benefits and barriers had significantly larger effect sizes for retrospective studies and severity had smaller effect sizes as compared to prospective studies. This suggests that when people think retrospectively about their health decisions, they perceive that benefits and barriers played a more important role and severity played a less important role than when they made the decision.

Both meta-analyses support the significance of susceptibility, severity, benefits, and barriers in predicting health behaviors. While Harrison et al. (1992) found only small effects for these components, several characteristics of the study might account for these findings (Sheeran \& Abraham, 1996). First, the researchers used extremely strict criteria for inclusion in the review and thus their results are based on a relatively small group of participants. Second, the reported effect sizes reflect each individual component rather than the model as a whole. The combined effects of the HBM components might be greater than the sum of the individual effects, however the researchers did not report this information. Finally, the effect sizes varied greatly across studies which influenced the overall average effect sizes. Therefore, these factors should be taken into consideration when interpreting the small effect sizes reported in the review.

While the HBM meta-analyses contain limitations, they also reveal problems in the HBM literature which should be addressed by future research. One of the most 
problematic aspects of the research on the HBM involves inconsistent measurement of HBM concepts (Harrison et al., 1992; Strecher et al., 1997). The large majority of studies use different ways to operationalize the constructs and fail to establish reliability or validity prior to testing the model. Even when studies use valid and reliability measures, these measures must be re-evaluated with each data collection since the psychometrics may change depending on the sample characteristics (Strecher et al., 1997). In addition, the review by Harrison et al. (1992) illustrates the infrequent inclusion of all four main components, much less the full HBM model. When the four components are incorporated, however, the relationships between these factors still remain unclear. Some researchers include these variables as independent predictors, whereas others test for moderation and mediation effects. Strecher et al. (1997) argues that the relationships among the HBM constructs represent complex, indirect associations rather than simple collections of variables. A study by Ronis and Harel (1989) highlighted this concept when they found that benefits mediated the effects of severity on breast examination behaviors. Moreover, Harrison et al. (1992) maintains that studies must develop weights and interaction terms to show how the four dimensions work together. In addition, the HBM is a cognitive model and relies on people to make logical and rational decisions. However, the cues to action component relies less on rational decision-making and represents a more complex factor in the model. Finally, the HBM only measures psychosocial variables which limit its ability to predict individual behaviors and can produce small effect sizes (Rosenstock, 1990). The next section of the review will address why the HBM still represented a good model for this study despite these limitations. 


\section{HPV Vaccine Acceptance}

Despite the limitations of previous research, the HBM is one of the only models that has been used in the sparse vaccination behavior literature. Perceived susceptibility, perceived severity, perceived effectiveness of the vaccine, barriers, and cues to action have been found to significantly predict influenza vaccination behavior (Brewer, Chapman, Gibbons, Gerrard, McCaul, \& Weinstein, 2007; Chapman \& Coups, 1999). The literature suggests that the HBM is also a useful model for HPV vaccine acceptability. A review by Brewer and Fazekas (2007) used the HBM to identify predictors of HPV vaccine acceptability in studies from 1995 through 2006. The researchers discovered 28 studies that met review criteria, all of which were conducted in the US prior to federal approval of the vaccine and none of which examined actual vaccine uptake. Most of the studies utilized cross-sectional designs and included small samples of parents and adults. The results indicate that higher perceived susceptibility of HPV infection and of getting cervical cancer was related to vaccine acceptability. On the other hand, higher perceived severity of HPV infection was not related to HPV vaccine acceptability, even though cervical cancer was perceived to have severe consequences. The review only explored one benefit, vaccine effectiveness, but found an association between higher perceived vaccine effectiveness and greater HPV vaccine intentions. Vaccine effectiveness referred to prevention of HPV infection, however none of the studies explored perceived effectiveness against genital warts or cervical cancer. Barriers such as low perceived vaccine safety, cost of the vaccine, and anticipated side effects predicted lower HPV vaccine acceptability. Finally, cues to action included school 
mandates and physician recommendations to get the vaccine, both of which were associated with higher vaccine acceptance.

Cross-sectional studies are highly prevalent within the HBM literature as indicated by the 22 out of 28 studies in the review that used this type of design. In a separate meta-analysis by Brewer et al. (2007) that examined risk perception and vaccination behavior, 28 out of 34 studies were cross-sectional. One concern with the cross-sectional design is the post hoc justification that can occur where participants inflate their sense of perceived severity, susceptibility, and benefits and deflate perceived barriers to justify their health behavior. As indicated previously, Harrison et al. (1992) found that benefits and barriers had significantly larger effect sizes for retrospective studies as compared to prospective studies. However, Harrison et al. (1992) and Brewer et al. (2007) found that effect sizes for severity and susceptibility were no greater in cross-sectional/retrospective than prospective vaccination studies, and in fact were smaller. This suggests that post hoc justification doesn't necessarily occur for severity and susceptibility, but might occur for benefits and barriers in cross-sectional designs.

The current study employed a cross-sectional design in order to compare HPV vaccinated women with non-HPV vaccinated women. Allen et al. (2009) conducted the only other known study to explore predictors of HPV vaccination status. Previous studies have generally focused on HPV vaccination intentions or acceptance as indicated in the review by Brewer and Fazekas (2007). The current study also explored HPV vaccination intentions among unvaccinated women, however the comparison of vaccinated and unvaccinated women provided important information not included in the previous literature. In order to study these differences, a cross-sectional design was employed. 


\section{College-Age Women and the HPV Vaccine}

Out of the 28 studies in the review of HBM predictors on HPV attitudes and vaccine acceptability, only three explored components of the HBM in college students (Boehner et al., 2003; Ramirez, Ramos, Clayton, Kanowitz, \& Moscicki, 1997; Yacobi, Tennant, Ferrante, Pal, \& Roetzheim, 1999). While these three studies provide a basis for future research, they contain several limitations. First, they failed to include all four of the main HBM concepts and only one focused specifically on college women whereas the others included both men and women. Since men and women may hold different beliefs about HPV and the vaccine, the results of these studies might not apply specifically to college women. Moreover, the HPV vaccine was not yet available at the time these studies were conducted. Therefore two of the studies explored attitudes toward HPV and one study explored acceptance of a hypothetical HPV vaccine. As such, these studies did not investigate acceptance of the currently available HPV vaccine. Several subsequent studies conducted after the review have explored acceptance of the Gardasil vaccine in college women, however, the paucity of research in this area remains surprising given the high prevalence of HPV in this population.

According to the existing literature, perceived susceptibility to HPV infection is relatively low among undergraduates (Gerend \& Magloire, 2008; Jones \& Cook, 2008; Yacobi et al., 1999). One study found that only $21 \%$ of college students believed they were at risk for HPV infection (Yacobi et al., 1999). However, this number rose to 58\% among sexually active women, suggesting that the risks of sexual activity become more salient once one becomes sexually active (Ramirez et al. 1997). Research indicates that lack of perceived susceptibility is associated with low vaccine acceptance, whereas, high 
perceived susceptibility is associated with increased intention to receive the vaccine (Gerend \& Magloire, 2008; Jones \& Cook, 2008; Boehner, 2003).

The finding that most college students do not perceive themselves to be at risk for HPV is concerning in light of the high prevalence of HPV infection in this population. A study by Ramirez et al. (1997) confirmed that college students' perceived susceptibility was unrelated to their actual risk as indicated by an HPV positive test. Women who did not perceive themselves at risk had almost identical prevalence rates as those who did. Even more concerning, low perceived susceptibility might inhibit college women's decision to receive the vaccine. However, it remains unknown to what extent this factor influences HPV vaccination. Determining this information will be important for assessing whether HPV vaccine health messages and interventions should emphasize HPV prevalence rates in college-age students.

Perceived severity of HPV infection among college women has been explored in a limited number of studies. The preliminary results suggest that the majority of college students would be at least somewhat upset if infected with HPV (Boehner et al., 2003; Jones \& Cook, 2008). While perceived severity did not significantly predict HPV vaccine acceptance in several studies, Kahn, Rosenthal, Jin, Huang, Namakydoust and Zimet (2008) found that higher perceived severity of HPV and HPV-related disease was positively associated with intention to receive the vaccine (Boehner et al., 2003; Jones \& Cook, 2008). Interestingly, one study that examined the stages of HPV vaccine adoption found lower perceived severity in college women who were undecided or contemplating the vaccine as compared to those who had decided against it (Allen et al., 2009). This suggests that a complex relationship could exist between HPV vaccination and perceived 
severity. Previous research indicates that benefits might mediate the effects of severity on health behavior, however this relationship has not been explored for HPV vaccination (Ronis \& Harel, 1989). Given the severe consequences that can result from HPV infection and the lack of research in this area, investigating the psychosocial factors that lead to refusal of the vaccine is important.

Only one study to the author's knowledge has explored the direct effects of perceived benefits on HPV vaccination in college women. As indicated previously, Allen et al. (2009) categorized college women according to their stage of HPV vaccine adoption using the transtheoretical model of change (Prochaska \& DiClemente, 1984). They found that women who planned to be vaccinated within the next 30 days (preparation stage) or were already vaccinated (action stage) had higher perceived benefit scores than those in the precontemplation and contemplation stages. Benefit scores were based on the vaccine's potential to stop the spread of HPV to partners and to prevent cervical cancer and genital warts. Another study on women aged 18 to 30 years found that most participants believed that getting the HPV vaccine would be a good idea, would be safe, would prevent cervical cancer and would prevent future acquisition of HPV (Kahn et al., 2003). Since only one study has investigated the association between perceived benefits and HPV vaccination among college-age women, the current study explored whether this factor influenced the uptake of the vaccine.

As acknowledged previously, certain barriers can influence HPV vaccine acceptability among women. According to the literature, potential barriers to women receiving the vaccine might include cost of the vaccine, not knowing enough about the vaccine, fears that others might consider them promiscuous and concerns about the safety 
of the vaccine (Constantine \& Jerman, 2007; Friedman \& Shepeard, 2007; Grant et al., 2009). However, very few studies have explored barriers to HPV vaccination in collegeage women. Preliminary results on college students suggest that those who have lower perceived barriers are more likely to accept the vaccine. For instance, students who weren't bothered by shots, believed that the vaccine was safe, or believed it was low cost had greater vaccine acceptance (Boehner et al., 2003). Conversely, a separate study found that having to pay $\$ 50$ for the vaccine made $63 \%$ of participants much less likely to accept the vaccine (Jones \& Cook, 2008). Finally, results indicate that women previously vaccinated have significantly lower perceived barriers than those who decided against vaccination (Allen et al., 2009).

Assessing barriers to vaccination represents an important area of exploration since these factors could inhibit wide-spread receipt of the vaccine. As previous studies indicate, college women possess positive attitudes towards the HPV vaccine. However, if this population experiences factors that inhibit their ability to receive the vaccine, then not as many college women will become vaccinated. Understanding these barriers represents the first step in successfully implementing the HPV vaccine. Once the barriers have been identified, then future research can explore methods for overcoming these factors.

Although cues to action are part of the full HBM, a limited number of studies have explored this construct. To the author's knowledge only one study has investigated cues to action for the HPV vaccine in college women. Jones and Cook (2008) found that knowing a close friend or relative with HPV predicted intention to receive the vaccine. In addition, the availability of a free vaccine and a doctor's recommendation for the vaccine 
made participants much more likely to accept vaccination. This suggests that external cues are particularly salient for trigging vaccination behavior. However, cues that might discourage vaccination, such as knowing someone who had negative side effects from the vaccine, have not been studied. One study found that most college students heard about HPV through public media like TV, magazines, and radio (Gerend \& Magloire, 2008). Campaigns have been advertising the HPV vaccine since it became available, however, it remains unknown what effect this media has on vaccination behavior. Moreover, Jones and Cook (2008) focused on external cues, but internal cues might also play a role in women's decision to receive the vaccine. Since preliminary results suggest that cues to action influence HPV vaccination, future studies should explore a larger variety of triggers.

Self-efficacy has been part of the HBM model ever since Rosenstock et al. recommended adding it in 1988 . However, a surprising number of studies fail to include this construct when testing the HBM. The original HBM model included perceived barriers, benefits, severity and susceptibility, whereas the expanded model incorporates cues to action as well as self-efficacy. The few studies that have investigated college student's self-efficacy to receive the HPV vaccine found that this construct significantly predicted vaccination intentions. In one study, self-efficacy uniquely predicted college women's intentions to receive the vaccine as well as mediated the effects of message framing (Gerend, Shepherd, \& Monday, 2008). A loss-framed message for a hypothetical one-shot HPV vaccine promoted the greatest self-efficacy and therefore the highest vaccine intentions. Moreover, a separate study on college men found that higher selfefficacy to receive a hypothetical HPV vaccine predicted vaccine acceptance (Gerend \& 
Barley, 2008). While these studies examined hypothetical HPV vaccines, Buchanan (2008) investigated the association between self-efficacy and the currently available Gardasil vaccine. The results indicate that even after including constructs from both the HBM and the Theory of Planned Behavior, self-efficacy remained a significant predictor of college women's intentions to receive the vaccine. Therefore, women's belief in their ability to overcome the necessary barriers to receive the three HPV vaccine injections appears to play an important role in predicting HPV vaccination behavior.

According to the limited number studies, the HBM appears to be a useful predictor of HPV vaccination intentions and vaccination status among college-age women. This study aimed to build upon this previous research and address some of the limitations in the literature. Most of the previous studies included only a few of the major HBM components instead of testing the full model. Even the studies that incorporated perceived susceptibility, perceived severity, benefits and barriers did not include other important components such as self-efficacy and cues to action. Thus, the HBM has not been adequately tested or operationalized within the HPV literature.

In addition, most of the studies were conducted prior to US federal approval of the vaccine and only one examined actual vaccine uptake (Allen et al., 2009). Finally, only a small number of HPV vaccine studies have focused on college-age women, which represents the most at-risk population. This study addressed some of these weaknesses by testing the full HBM model in vaccinated and unvaccinated women after the HPV vaccine had been available for three years. 


\section{Subjective Norms}

The HBM provides a useful framework for predicting health behavior, however, additional factors can also influence health decisions and should be explored as well. One factor that has been particularly influential among college-age women for receiving the HPV vaccine is subjective norms. This construct has traditionally been associated with the Theory of Planned Behavior and refers to how people important to the individual view the behavior (Fishbein \& Azjen, 1975). Subjective norms assess two different dimensions, injunctive and descriptive. Injunctive refers to whether an individual's social network expects and approves of the behavior and descriptive refers to whether the individual's social contacts perform the behavior themselves. Preliminary evidence indicates that the addition of this construct to the HBM could improve the ability to predict HPV vaccination. Studies incorporating aspects from both the HBM and the Theory of Planned Behavior found that subjective norms remained a significant predictor of women's intention to receive the HPV vaccine above and beyond the other constructs (Buchanan, 2008; Kahn et al., 2003).

According to the literature, college-age women seem to be especially influenced by the important people in their life. Participants who believed their parents, doctors, friends, and partners would encourage vaccination were more likely to accept the HPV vaccine (Boehner et al., 2003; Jones \& Cook, 2008; Costar, 2008). In one study, social norms (the perception that peers were planning to be vaccinated or already had been vaccinated) was the strongest predictor of HPV vaccine intentions, above and beyond the HBM constructs (Allen et al., 2009). College women who had already received the vaccine or were planning to receive the vaccine had significantly higher subjective norms 
as compared to women who decided not to get the vaccine. Therefore vaccinated women and those preparing to get the vaccine received more vaccination support from significant others. These results highlight the importance of assessing subjective norms for the HPV vaccine in college-age women. Although the majority of college-age women will likely decide for themselves whether to receive the vaccine, they may seek advice and support from the people they trust. The behavior of peers and the approval of important people may be one of the most significant determining factors in whether a woman becomes vaccinated.

\section{HPV Knowledge}

HPV knowledge has been a popular area of exploration even before the development of the HPV vaccine. Of the 28 studies in Brewer et al.'s (2007) review, 15 assessed knowledge and awareness of HPV. Most of these studies focused on adult women, however knowledge of HPV among college students has received substantial research attention. Awareness of HPV has appeared to increase over the last decade, perhaps as a result of publicity surrounding the HPV vaccine. One study from 1999 found that only $38 \%$ of college students had ever heard of HPV (Yacobi et al., 1999). However later studies found that more than $75 \%$ of college students had heard of HPV and $95 \%$ of college women had heard of genital warts (Gerend \& Magaloire, 2008; Baer, Allen, \& Braun, 2000). While current awareness of HPV is relatively high in this population, knowledge about the virus remains modest at best. HPV knowledge was particularly low in earlier studies, with average scores below the $68^{\text {th }}$ percentile in one study and a median score of three on a 13-item scale in another study (Ramirez et al., 1997; Yacobi et al., 
1999). Moreover, college students indicated they knew the least about HPV and felt that this STD had received the least amount of educational effort.

Knowledge scores increased slightly in later studies, with participants answering $45 \%$ of the questions correctly in one study, however, misunderstandings about HPV still persist (Lambert, 2001). The research indicates that most students are unaware that HPV can be silent, and can cause complications such as genital warts, although $92 \%$ correctly identified the causal link between HPV and cervical cancer (Yacobi et al., 1999; Baer et al., 2000; Allen et al., 2009; Gerend \& Magaloire, 2008). Knowledge about the transient nature of most HPV infections, the role of Pap smears in HPV detection, and the lack of available HPV treatment remained low (Allen et al., 2009; Gerend \& Magaloire, 2008). However, the most consistent misunderstanding across studies was the modes of transmission for both genital warts and HPV (Allen et al., 2009; Yacobi et al., 1999; Baer et al., 2000). The lack of knowledge regarding transmission is particularly disturbing since college-age students may unknowingly expose themselves to infection. For instance, students may hold the false assumption that using condoms will fully protect them against any STD including HPV. This false sense of security could lead to riskier sexual behaviors.

Findings have been mixed regarding which factors predict HPV knowledge. One study found that sexually experienced college women possessed higher knowledge scores than sexually inexperienced college women (Ramirez et al., 1997). However, another study found that sexual behaviors among college students, such as having multiple partners and not using condoms, predicted lower HPV knowledge and awareness (Yacobi et al., 1999). Therefore sexual behavior appears to be associated with HPV knowledge, 
however the direction of the association remains unclear. In addition, few studies have explored whether an association exists between HPV knowledge and health beliefs. Perceived risk of HPV infection was not significantly associated with HPV knowledge in one study, however, further research is needed to explore the association between knowledge and the HBM constructs (Ramirez et al., 1997). Finally, college women appear to possess greater awareness and knowledge of HPV than college men, perhaps as a result of the HPV vaccine and its media campaign targeting women (Gerend \& Magaloire, 2008).

Several studies have attempted to determine whether interventions can increase HPV knowledge and thereby enhance vaccine acceptance. Methods such as HPV educational interventions and information sheets have been found to heighten knowledge. A brief educational intervention on HPV transmission, prevention, and complications increased HPV knowledge by 34\% among college women (Lambert, 2001). In addition, detailed information sheets that describe the epidemiology and potential clinical sequelae of HPV infection increased HPV knowledge relative to the control group (Dempsey, Zimet, Davis, \& Koutsky, 2003). Information pamphlets regarding the effectiveness of the HPV vaccine on reducing HPV infection and thereby cervical cancer also heightened knowledge about the HPV vaccine (Chan, Cheung, Lo, \& Chung, 2007). While these interventions improved HPV knowledge, this increased awareness has not been directly associated with HPV acceptance. Of the studies that used the HPV information sheets, Davis, Dickman, Ferris, Dias (2004) found greater vaccine acceptance and, Dempsey et al. (2006) did not. However, Davis et al. (2004) did not measure changes in HPV knowledge, therefore the results cannot be attributed to increased knowledge. Chan et al. 
(2007) found higher acceptance rates after parents received an HPV vaccine information pamphlet, however increased acceptance was only associated with one knowledge item. Therefore, increasing HPV knowledge through interventions does not necessarily lead to vaccine acceptance. Moreover, most of the intervention studies have focused on parental acceptance of the vaccine for their daughters. As a result, it remains unknown whether HPV interventions would increase vaccine acceptance among college-age women.

A substantial number of studies have included HPV knowledge as the key predictor of HPV vaccine acceptability, however, the findings from this research remain inconsistent. Several studies found that HPV knowledge was unrelated to acceptance of the vaccine among college students (Boehner et al., 2003; Gerend \& Magaloire, 2008). Other studies have found a significantly positive relationship between HPV knowledge and vaccine acceptance. For instance, Jones and Cook (2008) discovered that college women who correctly answered two or three knowledge questions were eight times more likely to accept the vaccine than those who answered zero to one questions correctly. In addition, HPV knowledge appears to be positively associated with receiving the vaccine. One study found that HPV vaccinated women had significantly higher knowledge scores than those who decided against vaccination (Allen et al., 2009).

Differences in the HPV knowledge measures might account for some of the variance in the findings. The number of knowledge items, topics covered, and level of difficultly varied greatly between studies (Boehner et al., 2003; Gerend \& Magaloire, 2008; Jones \& Cook, 2008; Allen et al., 2009). Moreover, preliminary results suggest that there might be a difference between knowledge of the vaccine and knowledge of HPV. Costar (2008) found that college women's reported knowledge of the vaccine was 
significantly correlated with intention to become vaccinated, but their reported knowledge of HPV was not. Thus, future studies should differentiate between participants' knowledge of HPV and their knowledge of the HPV vaccine. Since the role of knowledge remains unclear, additional research is required to determine extent that this construct contributes to college-age women's decision to receive the HPV vaccine.

\section{Sexual Risk Factors}

Since HPV is a sexually transmitted disease, certain sexual risk factors increase an individual's chance of contracting the infection and are important to include in studies examining attitudes toward vaccines. As indicated previously, having a high number of lifetime sexual partners and engaging in intercourse at an early age increase the risk of HPV. While perceived susceptibility has been found to predict acceptance of the HPV vaccine, some studies have also explored whether actual risk for HPV increases the chances of receiving the vaccine. The findings indicate that sexually active college women and those with a higher number of sexual partners had higher intentions of getting the HPV vaccine (Gerend \& Magloire, 2008; Jones \& Cook, 2008; Boehner et al., 2003; Costar, 2008; Buchanan, 2008). Participants who reported more than five partners were four times more likely to intend to receive the vaccine than those with no partners (Jones \& Cook, 2008). One study did not find an association between sexual experience and vaccine acceptance, however this study used a hypothetical vaccine since the HPV vaccine was not yet available (Boehner et al., 2003). Therefore, women seem to be aware that sexual behavior increases their risk of contracting HPV and seek to protect themselves through the vaccine. Interestingly, women who were not yet sexually active expressed the least amount of interest in the vaccine (Gerend \& Magloire, 2008). This 
represents an important finding since the vaccine targets sexually inexperienced females and research indicates that this population can benefit the most from vaccination. These women might not perceive themselves at risk and therefore feel that the vaccine is unnecessary. Moreover, as opposed to adolescent girls, college-age women are more likely to make their own decision about becoming vaccinated.

Additional behaviors related to sexual activity also predict higher acceptance of the HPV vaccine. Several studies found that condom use with a committed partner as well as condom use with a new partner predicted higher intentions to get vaccinated against HPV (Costar, 2008; Buchanan, 2008). In addition, women who had been tested for HIV were more likely to accept the HPV vaccine (Gerend, Lee, \& Shepherd, 2006). This suggests that women who tend to engage in health-protective behavior have higher vaccination intentions. While contracting HPV depends upon an individual's sexual behavior, it also depends upon the partner's sexual history. For instance, if a male partner is uncircumcised, has an STD, or has had a higher number of sexual partners, this increases a woman's risk for HPV. However, research has not explored whether an association exists between a partner's sexual behavior and vaccine acceptance. If women are aware that their partner's sexual history can increase their risk of HPV, this might make them more likely to become vaccinated. Studies have not explored other sexual risk factors such as age of first intercourse and frequency of vaginal sex. These factors might also have an influence on women's intentions to receive the vaccine. While women seem to be aware that sexual behaviors can increase their risk of HPV infection, one study found that perceived susceptibility was unrelated to actual risk (Ramirez et al., 1997). 
Therefore the association between perceived risk, actual risk, and vaccine acceptance requires further research.

\section{Summary}

Few studies have explored factors that could influence HPV vaccination among college-age women. When studies have researched this topic, they have generally grouped college-age men and women together in one sample or used a hypothetical HPV vaccine instead of the currently available Gardasil vaccine. The HBM provides a useful framework for exploring HPV vaccination intentions and vaccination status and has been viewed by many researchers as an intuitively appealing model of health-related behaviors. However the limitations of this theory reside in its operationalization since many studies have not adequately measured the HBM or tested the model in its entirety. This study aimed to address some of these limitations by exploring the relationship between the full HBM model and college-age women's intentions to receive the HPV

Gardasil vaccine. In addition, the study used the HBM to examine differences between vaccinated and unvaccinated women. Finally, other factors that could influence vaccination, such as subjective norms, HPV knowledge and sexual risk factors were explored. 


\section{Chapter 3}

\section{Statement of the Problem}

Research on preventative health has focused on the factors that influence people's decision to engage in health behaviors. This study used the Health Belief Model as well as additional constructs to explore how HPV vaccinated college-age women differ from college-age women who haven't been vaccinated. These same constructs were used to predict college-age women's intentions to receive the HPV vaccine if they had not already been vaccinated. The original HBM framework includes perceived susceptibility, severity, benefits and barriers. The constructs of cues to action, self-efficacy and subjective norms were used to augment the original model. In addition, HPV knowledge and sexual risk factors for HPV were used to explore intentions to receive the vaccine as well as the difference between vaccinated and unvaccinated college-age women.

The human papillomavirus is a widespread virus that can have serious health consequences such as genital warts and anogenital cancers (National Cancer Institute, 2008; Souza et al., 2007). Women are particularly affected since HPV has been identified as the cause of cervical cancer, an illness developed by almost 12,000 women in the U.S (U.S. Cancer Statistics Working Group, 2009). While up to $75 \%$ of sexually active adults will become infected with HPV in their lifetime, college-aged women are particularly susceptible since a high rate of new infections occurs in this age group (Cates, 1999; Koutsky, 1997; Cervical Cancer, 1996; Bauer et al., 1991; Weinstock et al., 2004). The availability of a vaccine that protects against high risk strains of HPV has the potential to reduce HPV infection among women and prevent the spread of the virus to sexual partners. In order for the vaccine to successfully reduce the incidence of HPV, females 
must choose to become vaccinated. Although parents will likely play a role in deciding whether or not their daughters receive the vaccine, college-aged females will likely have more autonomy than younger females in making this decision. Therefore, understanding which factors influence women in this age group to receive the vaccine is an important step in creating a successful vaccination program.

The Health Belief Model serves as a useful framework for understanding factors that influence health decisions. Original components of the HBM, including perceived susceptibility, severity, benefits and barriers, have been widely used to predict a range of health preventative behaviors. For instance, the HBM significantly predicted influenza vaccination in several studies (Brewer et al., 2007; Chapman \& Coups, 1999). Research suggests that the HBM is also a useful model for HPV vaccine acceptability. In a review of 28 HPV studies, Brewer and Fazekas (2007) found that most of the HBM components predicted acceptance of the HPV vaccine. While several studies have used the HBM to explore college-age women's attitudes and beliefs regarding the HPV vaccine, many have used only parts of the model rather than all four main components. The exclusion of one of these components could result in the omission of potentially important information regarding women's decision to become vaccinated. This study aimed to test all four main components of the HBM.

Aside from the original HBM components, research indicates that other factors, such as cues to action, self-efficacy and subjective norms, can also influence health decisions. Several HBM studies have incorporated cues to action in order to augment the predictive utility of the original theory. For instance, both positive and negative cues have been found to significantly influence flu vaccination behavior (Cummings et al., 1979; 
Aho et al., 1979). One study explored cues to action specific to the HPV vaccine and discovered that certain cues increased intentions to become vaccinated (Jones \& Cook, 2008). Given that the HPV vaccine has been featured in media advertisements, cues to action seem particularly relevant for this health decision. Ever since Bandura introduced the concept in the 1970's, self-efficacy has been included in numerous HBM studies (Stretcher et al., 1997). Preliminary research on the HPV vaccine suggests that selfefficacy represents an influential factor in deciding whether to become vaccinated. Selfefficacy significantly predicted intentions to receive the vaccine in two studies on hypothetical HPV vaccines and one study on the Gardasil vaccine (Gerend \& Magliore, 2008; Gerend et al., 2008; Buchanan, 2008). While cues to action and self-efficacy have been incorporated into relatively few studies on the HPV vaccine, subjective norms represents a more common factor associated with this area of study. Perceptions of what important others believe about the HPV vaccine appear to greatly influence college-age women's decision to get vaccinated. For instance, acceptance of the vaccine was higher when parents, doctors, friends, and partners approved of the vaccine (Boehner et al., 2003; Jones \& Cook, 2008; Costar, 2008). According to the literature, cues to action, self-efficacy, and subjective norms are all factors that can influence women's decision to receive the HPV vaccine. Since very few studies have explored these factors along with the original HBM framework, this study augmented the model with additional components.

In addition to the aforementioned areas of interest, the present study collected descriptive data about the women in the sample. Specifically, women's HPV knowledge and sexual risk factors for HPV infection were collected to explore how these factors 
might contribute to HPV vaccination decisions. Previous literature suggests that these factors have an influence on women's intentions to receive the HPV vaccine (Allen et al., 2009; Gerend \& Magloire, 2008; Jones \& Cook, 2008; Boehner et al., 2003; Costar, 2008; Buchanan, 2008). Open-ended questions were included regarding the most salient benefits and drawbacks to receiving the vaccine as well as what factors led to or kept participants from receiving the vaccine. Finally, the study included demographic information, such as subjects' ages, ethnicities and health insurance status.

The HPV vaccine became available in 2006 , therefore studies have only recently begun to include women who intend to or have already received the Gardasil vaccine. Prior to the Gardasil vaccine, intentions to receive hypothetical HPV vaccines served as the outcome measure for many HPV studies. While research on hypothetical vaccines provides useful information about health decisions, studies on the currently available Gardasil vaccine are more applicable to women's actual health behavior. Since the HPV vaccine has been available for just over three years, research can explore the differences between women who have received the HPV vaccine and those who have not. This remains an important area to study since the factors that influence intention to receive a hypothetical or real vaccine might be different than those that influence vaccination behavior. A recent survey of adolescent girls aged 13-18 found that $37 \%$ had received at least one HPV vaccine shot through 2008 (CDC, 2009). However, only $18 \%$ have received the full three shots. This information suggests that HPV vaccine implementation in this age group might be more successful for the first shot than the full vaccine series. It remains unknown whether this pattern also applies to college-aged women since few studies have explored the number of women in this age group to become vaccinated. 
Based on gaps in the literature and important factors that still require exploration, this study tested the following hypotheses and research questions in HPV vaccinated and unvaccinated college-age women. Among those who have not received the HPV vaccine, intentions to receive the vaccine in the future were assessed.

HPV Vaccination Intentions

Research Hypotheses

Hypothesis 1: HBM variables of perceived susceptibility, severity, benefits, barriers and self-efficacy will each contribute unique variance in predicting HPV vaccination intentions among unvaccinated women. Women who report greater perceived susceptibility to HPV, greater perceived severity of HPV, greater perceived benefits of HPV vaccination, fewer perceived barriers to HPV vaccination, and higher self-efficacy for receiving the HPV vaccine will report higher intentions to obtain the HPV vaccine.

Several studies have found that the original HBM concepts significantly predict HPV vaccination intentions among college students. Research indicates that higher perceived susceptibility and lower perceived barriers among college-age students is associated with increased intention to receive the HPV vaccine (Gerend \& Magloire, 2008; Jones \& Cook, 2008; Boehner, 2003). Although perceived severity has not been significantly associated with HPV vaccine acceptance in some studies, it was found to be a significant predictor of young women's intention to receive the vaccine (Boehner et al., 2003; Jones \& Cook, 2008; Kahn et al., 2003; Kahn et al., 2008). The relationship between perceived benefits and intentions to receive the HPV vaccine has not been specifically studied in college-age women. However, higher perceived benefits have been 
associated with higher intentions to receive the vaccine among adults and parents of adolescents (Davis et al., 2004; Dempsey et al., 2006; Brewer \& Fazekas, 2007; Zimet, Mays, Winston, Kee, Dickes, Su, 2000). Thus, perceived benefits were hypothesized to predict HPV vaccination intentions among college-age women as well. Self-efficacy was included in this hypothesis since it is often included in the Health Belief Model. Among the few studies that have investigated college student's self-efficacy to receive the HPV vaccine, results indicate that this variable significantly predicts vaccination intentions (Buchanan, 2008; Gerend et al., 2008; Gerend \& Barley, 2008). Moreover, self-efficacy remained a significant predictor even after accounting for the HBM components of perceived susceptibility, severity, benefits and barriers (Buchanan, 2008; Gerend \& Barley, 2008). Therefore, this construct was hypothesized to significantly predict HPV vaccination intentions.

\section{Hypothesis 2: There will be a positive correlation between HPV knowledge} and HPV vaccination intentions for unvaccinated women

Although a substantial amount of researched has explored the role of HPV knowledge in HPV vaccine acceptability, the findings remain inconsistent. Several studies found that HPV knowledge was unrelated to acceptance of the HPV vaccine among college students (Boehner et al., 2003; Gerend \& Magaloire, 2008). However, Jones and Cook (2008) found that college women who correctly answered two or three knowledge questions were eight times more likely to accept the HPV vaccine. While findings have been mixed, differences in the level of difficulty, number of questions and topics covered in the HPV knowledge questionnaires could account for the variation in results. The studies that did not find a significant association with receiving the HPV 
vaccine might have included less valid and reliable HPV knowledge scales. This study aimed to incorporate a valid and reliable HPV knowledge questionnaire that has been used in previous studies. Given the evidence that this construct can serve as a significant predictor, it was hypothesized that HPV knowledge would have a positive association with HPV vaccination intentions.

\section{Hypothesis 3: There will be a positive relationship between reported HPV} risk factors and HPV vaccination intentions for unvaccinated women.

Several studies have explored whether women who are at higher risk for HPV infection are more likely to receive the HPV vaccine. Risk for contracting HPV is determined by certain sexual behaviors such as the number of sexual partners and the frequency of condom use. Findings indicate that sexually active college women and those with a higher number of sexual partners had higher intentions of getting the HPV vaccine (Gerend \& Magloire, 2008; Jones \& Cook, 2008; Boehner et al., 2003; Costar, 2008;

Buchanan, 2008). Participants who reported more than five partners were four times more likely to intend to receive the vaccine those with no partners (Jones \& Cook, 2008). This suggests that women who are at greater risk for HPV infection have higher intentions of becoming vaccinated against HPV.

HPV Vaccination Status

Research Hypotheses

Hypothesis 4: The Health Belief Model (HBM) variables of perceived susceptibility, severity, benefits, barriers and self-efficacy will significantly distinguish vaccinated women from unvaccinated women. Specifically, vaccinated women will report greater perceived susceptibility to HPV, greater perceived 
severity of HPV, greater perceived benefits of HPV vaccination, fewer perceived barriers to HPV vaccination, and greater self-efficacy for vaccination as compared to unvaccinated women.

One study by Allen et al. (2009) has explored HBM components in terms of vaccination status among college women. They reported that previously vaccinated college women had significantly lower perceived barriers than those who decided against the vaccine. Moreover, women who had already received the vaccine or planned to receive the vaccine within the next 30 days had higher perceived benefits as compared to participants who decided against the vaccine, were undecided about vaccination and those not planning to be vaccinated in the near future. Although Allen et al. (2009) did not find that perceived susceptibility or severity significantly differentiated vaccinated versus unvaccinated college women, these variables were still included in the hypothesis. Perceived susceptibility and severity have both been found to predict intentions to receive the vaccine. In addition, Allen et al. (2009) did not include self-efficacy, but this variable has also been found to predict vaccine intentions (Buchanan, 2008; Gerend \& Barley, 2008). Since intentions tend to predict behaviors, it would make sense that perceived susceptibility, perceived severity and self-efficacy would also influence whether women become vaccinated. Moreover Allen et al.'s (2009) study represents the only known study to use HBM variables to explore vaccinated versus unvaccinated college women.

Therefore, it would be premature to eliminate variables based on the results of one study.

Hypothesis 5: Vaccinated women will report higher self-efficacy to receive the vaccine as compared to unvaccinated women. 
Although self-efficacy has only been studied in relation to intentions to receive the HPV vaccine, it was hypothesized that this variable would act in a similar way with HPV vaccination status. Higher self-efficacy has been found to predict higher HPV vaccination intentions (Buchanan, 2008; Gerend \& Barley, 2008). Therefore since selfefficacy is a significant predictor of vaccination intentions, it remains likely that women who have obtained the vaccine would report higher scores for this construct as compared to women who have not received the vaccine.

Hypothesis 6: Vaccinated women will report more positive cues to action for receiving the HPV vaccine as compared to unvaccinated women.

Although cues to action have only been studied in relation to intentions to receive the HPV vaccine, it was hypothesized that this variable would act in a similar way with HPV vaccination status. Since positive cues to action increase vaccination intentions, it remains likely that women who have obtained the HPV vaccine would report a higher number of positive cues to action as well. In addition, studies on the influenza vaccine suggest that cues to action are associated with vaccination behavior. Brewer et al. (2007) and Chapman and Coups (1999) discovered that cues to action significantly predicted which participants received the influenza vaccine. Thus, it was hypothesized that positive cues to action would significantly differentiate college-age women who have received the vaccine and those who have not.

\section{Hypothesis 7: Vaccinated women will report higher subjective norms than}

\section{unvaccinated women.}

Prior studies have reported that subjective norms significantly predict HPV vaccination status among college students. Allen et al. (2009) found that college women 
who planned to receive the vaccine sometime in the future and those who had already received the vaccine had significantly higher subjective norms than women who had decided against the vaccine. In addition, subjective norms remained one of the most significant predictors of vaccination status (Allen et al., 2009). As a result, college-age women who had received the HPV vaccine were hypothesized to have higher subjective norms than women who have not received the vaccine.

\section{Hypothesis 8: Vaccinated women will have higher HPV knowledge scores}

\section{than unvaccinated women.}

Although a substantial amount of researched has explored the role of HPV knowledge in HPV vaccine acceptability, only one study has explored this construct in relation to HPV vaccination status. Allen et al. (2009) found that college women who had already been vaccinated had significantly higher knowledge scores than those who had decided against vaccination. Given the evidence that this construct can serve as a significant predictor, it was hypothesized that vaccinated college-age women would have higher HPV knowledge scores than those who have not received the vaccine.

\section{Hypothesis 9: Vaccinated women will report higher levels of HPV risk factors}

\section{than unvaccinated women.}

While several studies have explored whether HPV risk factors predict HPV vaccination intentions, no study has used this construct to differentiate HPV vaccinated and unvaccinated women. However, research indicates that the presence of HPV risk factors, such as a high number of sexual partners, predicts higher intentions of getting the HPV vaccine (Gerend \& Magloire, 2008; Jones \& Cook, 2008; Boehner et al., 2003; Costar, 2008; Buchanan, 2008). Therefore, it was hypothesized that a greater number of 
HPV risk factors would be associated with women who have received the HPV vaccine as opposed to those who have not been vaccinated.

Research Questions

\section{Research Question 1: What is the prevalence of vaccinated women in the} sample?

A limited number of studies have researched HPV vaccination since the vaccine became licensed in the U.S. in 2006. Among the studies conducted after 2006, several have explored the rate of HPV vaccination among young women. Studies conducted in 2007 found that only $12 \%$ of college-aged women had received at least one vaccine injection (Allen et al., 2009; Grant et al., 2009). Moreover, research suggests that a fair number of girls and women do not complete the three shots needed for sufficient protection against HPV. A national survey of vaccination coverage of adolescents found that while $37 \%$ had initiated the HPV vaccine series, only $18 \%$ received the three shots needed for full protection (CDC, 2009). Although the vaccine is administered over the course of six months, this time frame does not seem to fully account for the discrepancy between those who initiate the vaccine series and those who complete it. A national survey has not been conducted among college-aged women, therefore, it remains difficult to paint an accurate picture of HPV vaccine uptake among this population. Moreover, studies have not explored the prevalence of HPV vaccination past 2007 among collegeaged women. Due to the lack of research in this area, this study proposed a research question rather than a hypothesis about the number of vaccinated college-age women 
Question 2: For vaccinated women, is there a significant correlation between the length of time since vaccination and the HBM components of perceived benefits, barriers, susceptibility, and severity?

Since retrospective reporting has been found to influence the perceived importance of HBM components, the length of time since vaccination was deemed an important variable to include. Perceived benefits and barriers were found to play a more significant role and perceived severity was found to play a less significant role as compared to prospective reporting. This suggests that participants who recently received the vaccine might report less biased responses than those who received the vaccine several years ago. Therefore, if a significant relationship exists between length of time since vaccination and the HBM components, time since vaccination should be controlled for in data analyses.

Question 3: For vaccinated women, is there a significant correlation between satisfaction with receiving the HPV vaccine and the HBM components of perceived benefits, barriers, susceptibility, and severity?

Satisfaction with receiving the HPV vaccine has not been measured, therefore it remains unknown how this factor might relate to the HBM variables. Dissatisfaction with vaccination might occur if the participant experiences negative side-effects or embarrassment from receiving the HPV vaccine. If participants' HBM perceptions match their satisfaction level (ie. high satisfaction combined with high benefits, susceptibility and severity, and low barriers), this might indicate that their expectations about the vaccine were fulfilled. However, if there is a disconnect between the HBM variables and 
satisfaction, this might suggest that participants had either a more positive or a more negative experience than they expected.

\section{Question 4: Do women who report higher risk factors for HPV report higher} perceived susceptibility to HPV?

Several studies have investigated whether perceived susceptibility relates to actual risk for contracting sexually transmitted infections (STIs). Ethier, Kershaw, Niccolai, Lewis, and Ickovics (2003) found that sexually experienced teenage girls perceived little or no chance that they would contract an STI in the following year. However, approximately $38 \%$ of the participants tested positive for an STI. STI history represented the only significant risk indicator related to perceived susceptibility. Another study found that females aged 16 to 18 years who always used condoms were less likely to think that they would get an STI than participants who never used condoms (Abel \& Brunton, 2005). In a third study, young adults had elevated perceived risk of chlamydial and gonococcal infection if they inconsistently or never used condoms, reported multiple partners in the past year, received a chlamydia or gonnorhea diagnosis in the past year, or reported current symptoms (Ford, Jaccard, Millstein, Bardsley, Miller, 2004). Research in this area indicate mixed results with some studies finding numerous STI risk factors related to perceived susceptibility and others finding very few.

Only one known study has explored the relation between perceived susceptibility to HPV and HPV risk factors. In the study, sexually experienced college women listed the following reasons for perceiving themselves at risk for HPV infection: not practicing safe sex, having sexual experience, having multiple partners, not knowing a partner's diseases, testing positive for other STDs, and having a previous history of HPV infection 
(Ramirez et al., 1997). Interestingly, perceived susceptibility was not related to HPV infection. Given the mixed results from the STI literature and the lack of studies specific to HPV, the current study proposed a research question regarding the association between number of risk factors to HPV and perceived susceptibility.

Question 5: How will women respond to the following open-ended questions: What do you view as the greatest benefits of getting the HPV vaccination for yourself?

What do you view as the greatest drawbacks of getting the HPV vaccination for yourself?

What factors keep you from getting vaccinated?

What factors led you to get vaccinated?

The inclusion of these open-ended questions could provide additional information about the most important factors that influence women's decision to get vaccinated. Moreover, this represents a way to assess aspects of women's decision-making that standardized measures might not capture. The Health Belief Model measures the rational aspects of decision-making and these questions could help determine whether rationality actually represents the most salient influential factor or whether non-rational aspects play a role. 


\section{Chapter 4}

\section{Methods}

\section{Design}

In order to explore the factors that influence HPV vaccination, a cross-sectional field design was utilized. Cross-sectional studies are highly prevalent within the HBM literature (Brewer et al., 2007). Although prospective studies are preferred since this type of design increases the likelihood that the HBM variables motivate the behavior, crosssectional studies are often more feasible, or may be the only feasible approach, and in any case can still provide useful information. For instance, researchers have been able to produce rigorous cross-sectional studies exploring the effect of HBM variables on preventative behaviors such as cervical screening uptake and MMR vaccination (Orbell, Crombie \& Johnston, 1996; Hamilton-West, 2006). In a vaccination meta-analysis by Brewer et al. (2007), each study received a quality score based on the study's methodological rigor. Many of the cross-sectional studies received high quality scores and a study by Hamilton-West (2006) that compared MMR vaccinated versus unvaccinated students received the highest quality rating. Therefore, cross-sectional studies can still possess sound methodologies and produce valuable findings.

The current study employed a cross-sectional design in order to compare HPV vaccinated women with non-HPV vaccinated women. A dichotomous measure of HPV vaccination status was used, with women who had obtained the vaccine falling into one group ("Yes") and women who had not obtained the vaccine in the other group ("No"). This outcome measure provided important information not included in the previous literature. The opportunity for HPV vaccination had been available for three years, so 
women who had not yet been vaccinated were hypothesized to be different from women who had been vaccinated within this time period. This study attempted to determine which factors differentiated women who had chosen to take a preventative health action from those who had not.

This study also explored HPV vaccination intentions among unvaccinated women. This outcome variable was measured on a Likert scale and was only included in the sample of women who had not received the vaccine. The predictor variables for both outcome measures included the HBM variables of perceived susceptibility, perceived severity, perceived benefits, perceived barriers, cues to action, and self efficacy. Knowledge of HPV, subjective norms for receiving the vaccine, and sexual risk factors for HPV infection also served as predictor variables. Logistic regression analyses were used on HPV vaccination status to determine how the different predictor variables related to this outcome measure. In addition, multiple linear regression analyses were conducted on HPV vaccination intentions.

\section{Participants}

The target population included college-aged women who were eligible to receive the HPV vaccine. The sample included 208 females between the ages of 18 and 26 since 26 is the age limit for the vaccine. Before beginning the study, potential participants were informed that the study explores the perceptions of female college-age students toward women's health involving a common sexually transmitted disease and its prevention. The participants were a convenience sample recruited from sororities and/or psychology and education classes from three universities in the Mid-Atlantic, one university in the Midwest and one community college in the West. Incentives such as class credit or the 
possibility of winning a $\$ 40$ gift certificate were offered in return for participation. Of the 232 participants who gave informed consent to take the online study, a total of 218 completed it, for a completion rate of $94.0 \%$. Six women were excluded from further analysis since they were older than age 26 and four participants were excluded since they did not give their age and may have been ineligible for the study. This left a total sample size of 208 participants. Of these women, 150 reported receiving the vaccine and 58 reported not receiving the vaccine. Missing values were replaced using the participants' mean score for that particular scale. A total of 51 missing values were calculated for the current sample. Of the participants who identified their university, 160 were from sororities or undergraduate/graduate psychology/education classes at the University of Maryland. Participants from other universities were recruited through specific classes that were taught by faculty members acquainted with the researchers. This was an attempt to obtain geographical diversity as well as age-related differences in the case of the graduate class, although the samples from these other universities were very small. For instance, 17 were from an undergraduate psychology class at Drake University, 14 were from a graduate psychology class at Towson University, 6 were from a community college psychology class at Mt. San Antonio College and 2 were from Columbia University. The rest of the participants did not identify their university.

The mean age was $20.97(\mathrm{SD}=1.44)$ for vaccinated participants and 21.24 $(\mathrm{SD}=1.49)$ for unvaccinated participants. Of the entire sample, participants represented at least 8 different ethnicities, although the majority of women were Caucasian (69.2\%). Most of the sample consisted of college students (89.0\%), with the greatest number in their fourth year of college (47.6\%), however, some of the participants had graduated 
from college $(6.7 \%)$ or were in graduate school (3.9\%). Comparable numbers of participants were single or in a relationship with $53.4 \%$ of women reporting being single and $43.8 \%$ of women reporting being in a committed relationship. For a more comprehensive picture of the participants' demographic information, see Table 1. The total percentages for where participants had heard of HPV and the HPV vaccine exceed $100 \%$ since participants could choose multiple options.

In terms of sexual activity, $80.0 \%$ of vaccinated participants reported being currently sexually active as compared to $53.4 \%$ of unvaccinated participants. Additional information about the participants' sexual risk factors is presented in Table 2. If participants answered "No" to current sexual activity, then they skipped all other questions about intercourse. Similarly, if participants answered "No" to penis/vagina intercourse or to penis/anal intercourse, then they skipped all other questions related to these topics. Therefore, not all questions have a sample size of 208 .

\section{Measures}

\section{Demographics}

A demographics questionnaire based on Costar's (2008) study was used to collect information about participants' backgrounds (see Appendix A). The questionnaire included items on participants' age, gender, ethnicity, current year in college, and romantic relationship status. Health information relevant to HPV was also collected including general insurance coverage, insurance coverage of the HPV vaccine, and frequency of gynecological check-ups over the last two years. In addition, several questions based on Allen et al.'s (2009) study assessed awareness of HPV and the HPV 
vaccine, including whether participants had heard of HPV and the HPV vaccine and how they had heard about it.

\section{Sexual Risk Factors}

Since HPV is a sexually transmitted disease, this study incorporated questions regarding sexual risk factors for HPV from previous studies (Costar, 2008; Winer et al., 2003; Winer et al., 2006) (see Appendix B). Participants were asked about their personal history with HPV, sexually transmitted diseases, abnormal Pap smears, STD tests, unintended pregnancies and cervical cancer. In addition, participants were asked about behaviors related to sexual activity, including penis/vagina and/or anal intercourse, age of first sexual activities, number of sexual partners, type of birth control used, and the frequency of condom use when in committed relationships and with new partners.

\section{Health Belief Model}

Valid and reliable scales for the Health Belief Model (HBM) are largely unavailable for most health behaviors. Several studies have developed instruments with sound psychometric properties for specific health concerns, such as breast selfexamination, hypertension regimens, and children's obesity regimens (Champion, 1984; Abraham \& Williams, 1991; Maimen, Becker, Kirscht, Haefner, \& Drachman, 1977). While these scales have been modified for use in different studies, it would be difficult to apply these scales to other health behaviors. For instance, constructs such as benefits, barriers and cues to action require behavior-specific measures (Strecher \& Rosenstock, 1997). Even when valid and reliable scales are available, these measures must be reevaluated with each data collection to determine the sample-specific psychometrics. Therefore, it is standard practice for researchers to create their own measures when 
utilizing the HBM in order to make the scales specific to a particular health behavior. To create their own measures, researchers draw on items used in previous studies and reword them to fit the health behavior being investigated.

Recent HBM studies focusing on the HPV vaccine have drawn from the previous HPV literature to create questionnaires. However, these studies have not focused on instrument development and many did not explore the construct validity or reliability of these measures. One study by Allen et al. (2009) tested the face validity of their measure by having experts in the field review the content and conducted cognitive interviews to ensure item comprehension and interpretability. The items in the measure assessed perceived severity, perceived susceptibility, perceived benefits, and perceived barriers for HPV vaccination in a sample of college females. Cronbach Alphas were not reported for the study, therefore the scale reliabilities are unknown. Due to the lack of psychometric data available for current measures, this study created an HBM scale by utilizing items tested by Allen et al. (2009) as well as following guidelines presented by Ronis (1992) and Brewer et al. (2007) for framing threat perception questions. In addition, previously validated and reliable HBM measures for breast self-examination behavior and cervical screening were used as a guide for item development (Champion, 1984; Orbell, et al., 1996).

\section{Perceived Susceptibility}

Ronis (1992) found that health behavior was more accurately predicted by measures of susceptibility that were made conditional on not taking action. As Brewer et al. (2007) asserts, unconditioned risk questions, particularly in cross-sectional analyses, will underestimate the relationship between risk perceptions and behavior. Participants 
who received the vaccine will interpret unconditioned measures of susceptibility differently than participants who did not receive the vaccine. Therefore, six items conditioned on inaction were used to measure vaccinated and unvaccinated participants' perceived susceptibility to HPV infection (see Appendix C). Three items measured a participant's perceived absolute risk of developing HPV and HPV-related diseases if not vaccinated. These items included questions such as, "What are your chances of contracting HPV if you didn't have the HPV vaccine?" Three items were related to perceived relative risk of developing HPV and HPV-related diseases if not vaccinated. These items included questions such as, "What are your chances of contracting HPV if you didn't have the HPV vaccine compared to other college-aged women?" All six items were rated on a $10 \%$ increment scale of $0-100 \%$. The percentages were averaged to create a final score ranging from $0-100 \%$ where low scores indicated low perceived susceptibility and high scores indicated high perceived susceptibility if not vaccinated. In the present study, Cronbach alpha's for this scale were .92 for both vaccinated and unvaccinated participants.

\section{Perceived Severity}

The perceived severity measure contained six items that assessed respondents' reactions to a potential diagnosis of HPV and concerns about potential consequences of infection (see Appendix C). The six items included statements such as, "I would be embarrassed to let others know that I have HPV if I found out that I contracted the disease," and "If I contracted HPV, it could harm my future health". Participants rated these statements on a 7-point Likert scale where 1 means "Strongly Disagree" and 7 means "Strongly Agree". The scores were summed to create a total score ranging from 6 
to 42 where low scores indicate low perceived severity and high scores indicate high perceived severity. In the present study, Cronbach alpha's for this scale were .73 for both vaccinated and unvaccinated participants.

\section{Perceived Benefits}

Six items were used to measure participants' perceived benefits of receiving the HPV vaccine (see Appendix C). The items included statements such as, "Getting Gardasil is important for my health" and "Gardasil is effective in preventing an HPV infection". These items were rated on a 7-point scale where 1 means "Strongly Disagree" and 7 means "Strongly Agree" and scores were summed together with total scores ranging from 6 to 42 . Low scores indicated low levels of perceived benefits and high scores indicated high levels of perceived benefits of receiving the HPV vaccine. The Cronbach alpha's for this scale were .81 for vaccinated participants and .83 for unvaccinated participants.

\section{Perceived Barriers}

Perceived barriers were assessed by participants' responses to three items about the perceived negative aspects of the vaccine (see Appendix C). The items referred to the stigma of receiving the vaccine and included questions such as, "If other people knew I received Gardasil, I would be embarrassed.” These items were rated on a 7-point scale where 1 means "Strongly Disagree" and 7 means "Strongly Agree". All three items were summed together with total scores ranging from 3 to 21 . Low scores indicated low levels of perceived barriers and high scores indicated high levels of perceived barriers to receiving the HPV vaccine. The Cronbach alpha's for this scale were .54 for vaccinated participants and .72 for unvaccinated participants. 


\section{Self- Efficacy}

To the author's knowledge, a validated self-efficacy scale for receiving the HPV vaccine does not currently exist. Self-efficacy refers to a woman's belief in her ability to overcome the necessary barriers to receive the HPV vaccine. Therefore, questions assessing self-efficacy for receiving the HPV vaccine were included after each question assessing barriers (see Appendix D). The items asked "Given your answer to the above question (\#__, how confident are you that you could get Gardasil?”. These questions were rated on a 7-point scale where 1 means "Not Confident" and 7 means "Very Confident". Since there were three barrier questions, there were three items assessing self-efficacy. These items were summed together to create total scores ranging from 3 to

21. Low scores indicated low levels of self-efficacy and high scores indicated high levels of self-efficacy for receiving the HPV vaccine. The Cronbach alpha's for this scale were .86 for vaccinated participants and .90 for unvaccinated participants.

\section{Cues to Action}

Cues to action refer to triggers either internally or within the environment that could potentially influence health behavior. These cues depend on the specific health concerns being investigated, therefore few validated measures of cues to action exist. As such, there is no known reliable or validated cues to action scale for the HPV vaccine. This study used 11 items that other HPV studies have employed that fit into the cues to action construct (Jones \& Cook, 2008; Buchanan, 2008; Allen et al., 2009) (see Appendix E). The items asked questions such as, "Have you ever known anyone who has had HPV?" and "Has a health care provider ever recommended that you receive the HPV vaccine (Gardasil)?" These questions were rated on a Likert scale where "Never" is 
equivalent to 1 point, " $1-2$ " is equivalent to 2 points, " $3-4$ " is equivalent to 3 points and " $5+$ " is equivalent to 4 points. The question "Have you ever known anyone who has had negative side effects from a vaccine?" was reverse coded. The items were summed to create a total score ranging from 11 to 44 with low scores indicating a lower number of positive cues to action and high scores indicating a higher number of positive cues to action for receiving the HPV vaccine. The Cronbach alpha's for this scale were .66 for vaccinated participants and .59 for unvaccinated participants. Other researchers have had difficulty obtaining high alphas for cues to action scales which may be due to the complex nature of the construct. This construct can include many different internal and external triggers which make it difficult to clearly define. In addition, the low alphas in this study may be due to the fact that items were included from a number of different studies and combined into one scale. Since the purpose of this study was not measure development, this scale was still included despite low internal reliability.

\section{Subjective Norms}

Subjective norms towards HPV vaccination were measured by six items based on a scale used in Costar's (2008) dissertation (See Appendix F). Scores from the measure produced high reliability in a sample of college women (Cronbach alpha of .87). Five of the items included statements such as, "If they knew about the HPV vaccine, most people whose opinions I value would approve/disapprove of me getting vaccinated against human papillomavirus (HPV)" and "If they knew about the HPV vaccine, my friends would approve/disapprove of me getting vaccinated against human papillomavirus (HPV)". Answers were rated on a Likert scale ranging from 1-7 where 1 means "Strongly Disapprove" and 7 means "Strongly Approve". One item included, "If they knew about 
the HPV vaccine, most women who are important to me would get themselves vaccinated against the human papillomavirus (HPV) if they were at risk" and was rated on a on a Likert 7-point scale where 1 means "Strongly Disagree" and 7 means "Strongly Agree." The items were summed to create total scores ranging from 6 to 42 where low scores represented perceived negative feelings toward HPV vaccination by important others and high scores represented perceived positive feelings toward HPV vaccination by important others. The Cronbach alpha's for this scale were .88 for vaccinated participants and .89 for unvaccinated participants.

\section{HPV Knowledge}

Knowledge about HPV and the HPV vaccine were assessed with nine items from Allen et al.'s (2009) study (see Appendix G). There were five multiple choice questions including items such as, "Which of the following health issues are related to HPV? (Please select all that apply.)" with the choices "Cervical cancer, Heart disease, HIV, Genital warts or I don't know”. There were also four true/false statements including items such as, "HPV affects only women" and "HPV can occur without symptoms". Participants received 1 point for each correct response and 0 points for any incorrect responses. For questions that had two correct responses, participants received .5 points for each correct response, with a total of 1 point if the participant gave both correct responses. Answers to each question were provided after participants responded. The items were summed to create total scores ranging from 0 to 9 where low scores indicated low HPV knowledge and high scores indicated high HPV knowledge. 


\section{Decision-making}

Two open-ended questions were used to assess the biggest influential factors in participants' decision about getting vaccinated (see Appendix H). The first question asked, "What do you view as the greatest benefits of getting the HPV vaccination for yourself?" and the second question asked "What do you view as the greatest drawbacks of getting the HPV vaccination for yourself?" In addition, vaccinated participants were asked the open-ended question, "What factors led you to get vaccinated?" and rated their level of satisfaction with their decision to receive the vaccine on a 7-point scale. Unvaccinated participants were asked the open-ended question, "What factors keep you from getting vaccinated?" and rated their level of satisfaction with their decision to not yet receive the vaccine on a 7-point scale.

\section{Outcome Variables}

HPV Vaccination Status. HPV vaccination status served as an outcome measure and included one multiple choice item used in Costar's (2008) dissertation (see Appendix I). This item asked "Which of the following best describes your current situation?" followed by the choices a. "Have completed the series of 3 shots for the HPV vaccine", b. "Have started, but not completed, the series of 3 shots for the HPV vaccine", c. "Have scheduled an appointment with my doctor to receive the HPV vaccine", d. "Have not received the HPV vaccine or scheduled an appointment to receive the HPV vaccine”. Choices a and b were coded as Group 1: Vaccinated. Choices $\mathrm{c}$ and $\mathrm{d}$ were coded as Group 2: Not Vaccinated. A similar item was previously used in a sample of university women (Costar, 2008). In addition, vaccinated participants were asked to respond to the 
open statement "Please indicate the approximate date of your last HPV vaccine shot" in order to assess the length of time since vaccination. Participants who indicated that they had not yet completed the vaccine series were asked if they planned to finish.

HPV Vaccination Intentions. Intentions to get vaccinated against HPV were assessed using four items, taken from Costar's (2008) dissertation (see Appendix J). The first item included the statement, "I want to get vaccinated against the human papillomavirus (HPV) within the next year," followed by "I expect that I will get vaccinated against the human papillomavirus (HPV) within the next year," "I do not intend to get vaccinated against the human papillomavirus (HPV) within the next year," and "I plan to make an appointment for the HPV vaccine within the next year."

Responses were rated on a 7-point scale where 1 means "Strongly Disagree" and 7 means "Strongly Agree". Items were summed together (the third item was reverse coded) for a total score ranging from 4 to 28 . Low scores indicated lower intentions to be vaccinated while high scores indicated higher intentions to be vaccinated. Costar (2008) used this scale in a sample of university women and found a coefficient alpha of .94. In the present study, the Cronbach alpha for this scale was .88 .

\section{Procedures}

College-age women in psychology or education classes and/or sororities at five different universities/colleges completed the measures through Psychdata, an online survey system. The website to the study survey was distributed to the participants along with a description of the study (see Appendix N). The study was advertised in conjunction with another study recruiting both men and women to explore HPV vaccine health messages. Therefore, the advertisement described studies exploring men and 
women's attitudes about sexual health. The men who participated were assigned to the other study along with half of the women who were not yet vaccinated. When participants went to the survey website, they viewed the informed consent which encouraged participants to complete the survey in one sitting in a private area with as few distractions as possible (see Appendix K). By clicking "continue" after the informed consent, participants gave their consent to participate in the study. At the end of the survey, participants were asked to leave an identifying number or their email address which was used to give them either class credit or enter them into the raffle for the gift certificate (see Appendix L). The participants' email addresses were not connected to their survey responses in any way and participants were assured that their information was kept confidential throughout the survey.

This study drew from previous research to create scales that measure perceived benefits, perceived barriers, perceived severity and perceived susceptibility, subjective norms, cues to action and self-efficacy. The psychometric properties of these scales were determined throughout the course of the study. Reliability (internal consistency) of the scales were calculated based on the scores of the participants in the study. Moreover, two experts in the field of HPV vaccination research assessed content validity by reviewing items from the HBM scale (Appendix C) and categorizing them according to the model constructs.

Participants completed the HPV Knowledge measure first so that participants' level of knowledge was unaffected by the rest of the survey. The outcome measure of HPV Vaccination was included next since the administration of subsequent measures depended on the vaccination status. The participants who indicated that they had not 
received the HPV vaccine completed the outcome measure of HPV Vaccination Intentions (see Appendix F) as well as questions about Decision-Making pertaining to unvaccinated women. Participants who indicated that they had received the HPV vaccine were asked for the date of their last shot as well as questions about Decision-Making pertaining to vaccinated women. The individual items measuring Perceived Benefits, Perceived Barriers, Perceived Susceptibility and Perceived Severity were randomly ordered and combined into one HBM scale to avoid redundancy (see Appendix C). Selfefficacy questions were included following each Perceived Barriers item. The HBM scale and predictor measures for Cues to Action, Subjective Norms and Sexual Risk Factors were then presented. Participants completed the demographics questionnaire at the end of the survey and had the opportunity to find out more information about HPV by clicking on links (see Appendix M). 


\section{Chapter 5}

Results

The results chapter includes preliminary analyses, sample description of demographics and sexual risk factors, preliminary correlations, exploratory factor analysis for the HBM variables and analysis of the nine hypotheses and five research questions.

\section{Preliminary Analyses}

The analyses were completed using the statistical package software SPSS Version 17. The analyzed variables were screened for missing values, and one missing value was found in the Knowledge Scale, five missing values were found in the Susceptibility Scale, three missing values were found in the Severity Scale, eight missing values were found in the Benefits Scale, nine missing values were found in the Barriers Scale, eight missing values were found for the Self-Efficacy Scale, 11 missing values were found for the Cues to Action Scale, five missing values were found for the Subjective Norms Scale, and one missing value was found for the Vaccine Intentions Scale, totaling 51 missing values. These missing values were replaced using the participant's mean score for that particular scale. Data from the 14 participants who did not complete two or more of the scales was discarded leaving a total sample of 208. The normality of each variable was checked and the scales were assessed for internal consistency. Subjective norms, selfefficacy, benefits and barriers displayed some skewness and/or kurtosis as indicated by values greater than 1 . However, these variables were not transformed since the assumptions of linear regression analyses were met, including normality, linearity, independence and homoscedasticity. All other variables were close to normally 
distributed. The open-ended questions were scored by 3 coders, who reached an acceptable reliability level ranging from a cohen's kappa of .76 to .94 . The coders reached consensus on the additional responses through discussion.

\section{Sample Description of Demographics and Sexual Risk Factors}

Descriptive data about the demographic and sexual risk factors of participants are presented in Tables 1 and 2. On some of the sexual risk questions the option of "prefer not to respond" was offered to participants as required by the IRB. The approximate percentage that did not respond to various items was low and can be seen in Tables 1 and 2. The mean age of the sample was $21.04(\mathrm{SD}=1.46)$ and all participants were 26 or under since this is the cutoff age at which women can receive the HPV vaccine. Since all of the participants were in the age range of 18-26 they were considered college-age and the majority of participants were undergraduates, however a small number were graduate students $(3.9 \%)$ or college graduates $(6.7 \%)$. The sample was primarily White $(69.2 \%)$, college students (89\%), approximately half of whom were in a committed relationship $(43.8 \%)$ and half of whom were single (53.4\%). Fewer unvaccinated women reported that they had health insurance (89.7\%) as compared to vaccinated women (97.3\%). Moreover, only $60.3 \%$ of unvaccinated women had health insurance that covered the HPV vaccine, as compared to $83.3 \%$ of vaccinated women. In terms of gynecological appointments, vaccinated participants reported an average of 2.0 visits $(\mathrm{SD}=1.44)$ over the past two years, whereas unvaccinated participants reported an average of 1.3 visits $(\mathrm{SD}=1.41)$. In addition, more unvaccinated women (36.2\%) reported that they had not attended a gynecological appointment in the past two years than vaccinated women $(12.0 \%)$. Vaccinated women had significantly more $\left(t_{204}=-3.27, p<0.001\right)$ gynecologists 
visits as compared to unvaccinated women. The number of visits ranged from 0-10 for the entire sample.

Most of the women had heard of HPV (97.6\%) and Gardasil (97.6\%) before taking the survey. Participants reported multiple ways of hearing about HPV, the most common for vaccinated women were through a health care provider (53.3\%), television or radio $(41.3 \%)$ and/or a family member $(38.0 \%)$, whereas the most common for unvaccinated women were through a health care provider (36.2\%), television or radio (50.0\%) and/or a friend (29.3\%). The most common ways that vaccinated participants had heard of Gardasil were through the television or radio (30.0\%), a health care provider $(25.3 \%)$ or through a family member $(28.0 \%)$. The most common ways that unvaccinated participants had heard of Gardasil were through television or radio (46.6\%) and a health care provider $(19.0 \%)$. For vaccinated women, the mean reported satisfaction with the decision to receive the vaccine was $6.18(\mathrm{SD}=1.44)$ with scores ranging from 1-7, where $1=$ low satisfaction and $7=$ high satisfaction. For unvaccinated women, the mean reported satisfaction with the decision to not yet receive the vaccine was $4.74(\mathrm{SD}=1.84)$ with scores ranging from 1-7. Therefore, vaccinated women were significantly $\left(t_{83.59}=-5.34\right.$, $p<0.001)$ more satisfied with their decision than unvaccinated women. Twenty-two of the vaccinated women had started but not completed all three shots. Among these women, four indicated that they do not plan to complete the vaccine series, 17 indicated that they do and one participant did not respond. Of the women who received the vaccine, the average time since the participant's last vaccination was 16.9 months $(\mathrm{SD}=11.0)$ with length of time ranging from 1 to 44 months. More details on demographic factors can be seen in Table 1. 
Most of the women reported being currently sexually active (72.6\%) and among these women, $93.7 \%$ reported having penis/vagina intercourse in the past. Vaccinated and unvaccinated women had the same mean age of first intercourse (17.3) and similar age ranges (13-22 for vaccinated women, 14-22 for unvaccinated women). However, the number of sexual partners differed by vaccination status, where vaccinated women had a mean of 5.16 partners $(\mathrm{SD}=4.58)$ and a range from $1-22$, whereas unvaccinated women had a mean of 3.72 partners $(\mathrm{SD}=3.25)$ and a range from 1-13. Vaccinated women had significantly more sexual partners than unvaccinated women $\left(t_{70}=-2.01, p<.05\right)$. While slightly more than half of the participants (59.5\%) reported always using a condom with a new partner, far fewer (19.4\%) reported always using a condom with a partner when in a committed relationship. A greater percentage of unvaccinated women reported always using a condom with a new partner and when in a committed relationship (respectively, $78.1 \%$ and $25.0 \%$ ) as compared to vaccinated women (respectively, 54.3\% and $17.9 \%$ ).

Among the women who were sexually active, relatively few (18.9\%) reported ever engaging in penis/anus intercourse. Only three (5.2\%) unvaccinated women reported engaging in anal intercourse. For women out of the total sample who had anal intercourse, the age of first anal intercourse ranged from 15-21 with a mean age of 18.6 $(\mathrm{SD}=1.71)$. The number of penile/anal sexual partners ranged from 1-5 with a mean of $1.62(\mathrm{SD}=1.08)$. Information on the sexual risk factors for participants is displayed in Table 2.

The means, standard deviation, and internal consistency values for the HBM measures for both vaccinated and unvaccinated women is presented in Table 3. The same information for the rest of the measures can be found in Table 4. 
Table 1. Demographic characteristics of participants $(\mathrm{N}=208)$

\begin{tabular}{lcccccc} 
& \multicolumn{2}{c}{ VACCINATED } & \multicolumn{2}{c}{ UNVACCINATED } & \multicolumn{2}{c}{ TOTAL SAMPLE } \\
\cline { 2 - 6 } Race/Ethnicity & $\boldsymbol{N}$ & Percentage & $\boldsymbol{N}$ & Percentage & $\boldsymbol{N}$ & Percentage \\
\hline African American/Black & 7 & $4.7 \%$ & 11 & $19 \%$ & 18 & $8.7 \%$ \\
Asian American/Pacific & 13 & $8.7 \%$ & 6 & $10.3 \%$ & 19 & $9.1 \%$ \\
Islander/ & & & & & & \\
Asian Indian/Pakistani & 3 & $2.0 \%$ & 4 & $6.9 \%$ & 7 & $3.4 \%$ \\
Middle-Easter/Arab & 1 & $0.7 \%$ & 1 & $1.7 \%$ & 2 & $1.0 \%$ \\
Biracial & 5 & $3.3 \%$ & 2 & $3.4 \%$ & 7 & $3.4 \%$ \\
Hispanic/Latino(a) & 7 & $4.7 \%$ & 2 & $3.4 \%$ & 9 & $4.3 \%$ \\
Native American/Native & 1 & $0.7 \%$ & 1 & $1.7 \%$ & 2 & $1.0 \%$ \\
Alaskan & & & & & & \\
White & 113 & $75.3 \%$ & 31 & $53.4 \%$ & 144 & $69.2 \%$
\end{tabular}

\begin{tabular}{lcccccc} 
& \multicolumn{2}{c}{ VACCINATED } & \multicolumn{2}{c}{ UNVACCINATED } & \multicolumn{2}{c}{ TOTAL SAMPLE } \\
\cline { 2 - 6 } Student Status & $\boldsymbol{N}$ & Percentage & $\boldsymbol{N}$ & Percentage & $\boldsymbol{N}$ & Percentage \\
\hline First Year & 5 & $3.3 \%$ & 1 & $1.7 \%$ & 6 & $2.9 \%$ \\
Second Year & 26 & $17.3 \%$ & 7 & $12.1 \%$ & 33 & $15.9 \%$ \\
Third Year & 33 & $22.0 \%$ & 11 & $19.0 \%$ & 44 & $21.2 \%$ \\
Fourth Year & 67 & $44.7 \%$ & 32 & $55.2 \%$ & 99 & $47.6 \%$ \\
Fifth Year & 1 & $0.7 \%$ & 2 & $3.4 \%$ & 3 & $1.4 \%$ \\
College Graduate & 10 & $6.7 \%$ & 4 & $6.9 \%$ & 14 & $6.7 \%$ \\
Graduate Student & 7 & $4,7 \%$ & 1 & $1.7 \%$ & 8 & $3.9 \%$ \\
No Answer & 1 & $0.7 \%$ & 0 & $0.0 \%$ & 1 & $0.5 \%$
\end{tabular}


Table 1 continued. Demographic characteristics of participants $(\mathrm{N}=208)$

\begin{tabular}{lcccccc} 
& \multicolumn{2}{c}{ VACCINATED } & \multicolumn{2}{c}{ UNVACCINATED } & \multicolumn{2}{c}{ TOTAL SAMPLE } \\
\cline { 2 - 7 } Relationship Status & $\boldsymbol{N}$ & Percentage & $\boldsymbol{N}$ & Percentage & $\boldsymbol{N}$ & Percentage \\
\hline Married & 4 & $2.7 \%$ & 2 & $3.4 \%$ & 6 & $2.9 \%$ \\
Committed Relationship & 62 & $41.3 \%$ & 29 & $50 \%$ & 91 & $43.8 \%$ \\
Single & 84 & $56.0 \%$ & 27 & $46.6 \%$ & 111 & $53.4 \%$ \\
& & & & & & \\
& & & & & \\
& & & & & \\
Health Insurance & $\boldsymbol{N}$ & Percentage & $\boldsymbol{N}$ & Percentage & $\boldsymbol{N}$ & Percentage \\
\hline Yes & 146 & $97.3 \%$ & 52 & $89.7 \%$ & 198 & $95.2 \%$ \\
No & 4 & $2.7 \%$ & 6 & $10.3 \%$ & 10 & $4.8 \%$
\end{tabular}

\begin{tabular}{lcccccc} 
& \multicolumn{2}{c}{ VACCINATED } & \multicolumn{2}{c}{ UNVACCINATED } & \multicolumn{2}{c}{ TOTAL SAMPLE } \\
\cline { 2 - 7 } Insurance for Vaccine & $\boldsymbol{N}$ & Percentage & $\boldsymbol{N}$ & Percentage & $\boldsymbol{N}$ & Percentage \\
\hline Yes & 125 & $83.3 \%$ & 35 & $60.3 \%$ & 160 & $76.9 \%$ \\
No & 17 & $11.3 \%$ & 12 & $20.7 \%$ & 29 & $13.9 \%$ \\
No Answer & 8 & $5.3 \%$ & 11 & $19.0 \%$ & 19 & $9.1 \%$
\end{tabular}

\begin{tabular}{lcccccc} 
& \multicolumn{2}{c}{ VACCINATED } & \multicolumn{2}{c}{ UNVACCINATED } & \multicolumn{2}{c}{ TOTAL SAMPLE } \\
\cline { 2 - 6 } Heard of HPV & $\boldsymbol{N}$ & Percentage & $\boldsymbol{N}$ & Percentage & $\boldsymbol{N}$ & Percentage \\
\hline Yes & 147 & $98.0 \%$ & 56 & $96.6 \%$ & 203 & $97.6 \%$ \\
No & 3 & $2.0 \%$ & 2 & $3.4 \%$ & 5 & $2.4 \%$
\end{tabular}


Table 1 continued. Demographic characteristics of participants $(\mathrm{N}=208)$

\begin{tabular}{|c|c|c|c|c|c|c|}
\hline \multirow[b]{2}{*}{ Where Heard of HPV } & \multicolumn{2}{|c|}{ VACCINATED } & \multicolumn{2}{|c|}{ UNVACCINATED } & \multicolumn{2}{|c|}{ TOTAL SAMPLE } \\
\hline & $N$ & Percentage & $N$ & Percentage & $N$ & Percentage \\
\hline Health care provider & 80 & $53.3 \%$ & 21 & $36.2 \%$ & 101 & $48.6 \%$ \\
\hline Friend & 21 & $14.0 \%$ & 17 & $29.3 \%$ & 38 & $18.3 \%$ \\
\hline Family member & 57 & $38.0 \%$ & 6 & $10.3 \%$ & 63 & $30.3 \%$ \\
\hline Television or radio & 62 & $41.3 \%$ & 29 & $50.0 \%$ & 91 & $43.8 \%$ \\
\hline Internet & 12 & $8.0 \%$ & 5 & $8.6 \%$ & 17 & $8.2 \%$ \\
\hline Newspaper or magazine & 18 & $12.0 \%$ & 9 & $15.5 \%$ & 27 & $13.0 \%$ \\
\hline At school & 26 & $17.3 \%$ & 12 & $20.7 \%$ & 38 & $18.3 \%$ \\
\hline I don't remember & 13 & $8.7 \%$ & 6 & $10.3 \%$ & 19 & $9.1 \%$ \\
\hline Other & 0 & $0.0 \%$ & 1 & $1.7 \%$ & 1 & $0.5 \%$ \\
\hline \multirow[t]{2}{*}{ No Answer } & 3 & $2.0 \%$ & 2 & $3.4 \%$ & 5 & $2.4 \%$ \\
\hline & \multicolumn{2}{|c|}{ VACCINATED } & \multicolumn{2}{|c|}{ UNVACCINATED } & \multicolumn{2}{|c|}{ TOTAL SAMPLE } \\
\hline Heard of Vaccine & $N$ & Percentage & $N$ & Percentage & $N$ & Percentage \\
\hline Yes & 150 & $100 \%$ & 53 & $91.4 \%$ & 203 & $97.6 \%$ \\
\hline No & 0 & $0 \%$ & 4 & $6.9 \%$ & 4 & $1.9 \%$ \\
\hline \multirow[t]{2}{*}{ I don't know } & 0 & $0 \%$ & 1 & $1.7 \%$ & 1 & $0.5 \%$ \\
\hline & \multicolumn{2}{|c|}{ VACCINATED } & \multicolumn{2}{|c|}{ UNVACCINATED } & \multicolumn{2}{|c|}{ TOTAL SAMPLE } \\
\hline Where Heard of Vaccine & $N$ & Percentage & $N$ & Percentage & $N$ & Percentage \\
\hline Health care provider & 38 & $25.3 \%$ & 11 & $19.0 \%$ & 49 & $23.6 \%$ \\
\hline Friend & 8 & $5.3 \%$ & 4 & $6.9 \%$ & 12 & $5.8 \%$ \\
\hline Family member & 42 & $28.0 \%$ & 5 & $8.6 \%$ & 47 & $22.6 \%$ \\
\hline Television or radio & 45 & $30.0 \%$ & 27 & $46.6 \%$ & 72 & $34.6 \%$ \\
\hline Internet & 0 & $0.0 \%$ & 0 & $0.0 \%$ & 0 & $0.0 \%$ \\
\hline Newspaper or magazine & 4 & $2.7 \%$ & 1 & $1.7 \%$ & 5 & $2.4 \%$ \\
\hline At school & 5 & $3.3 \%$ & 1 & $1.7 \%$ & 6 & $2.9 \%$ \\
\hline I don't remember & 6 & $4.0 \%$ & 4 & $6.9 \%$ & 10 & $4.8 \%$ \\
\hline Other & 1 & $0.7 \%$ & 0 & $0.0 \%$ & 1 & $0.5 \%$ \\
\hline No Answer & 1 & $0.7 \%$ & 5 & $8.6 \%$ & 6 & $2.9 \%$ \\
\hline
\end{tabular}


Table 2. Sexual risk factors $(\mathrm{N}=208)$

\begin{tabular}{lcccccc} 
& \multicolumn{2}{c}{ VACCINATED } & \multicolumn{2}{c}{ UNVACCINATED } & \multicolumn{2}{c}{ TOTAL SAMPLE } \\
\cline { 2 - 7 } Sexually Active & $\boldsymbol{N}$ & Percentage & $\boldsymbol{N}$ & Percentage & $\boldsymbol{N}$ & Percentage \\
\hline Yes & 120 & $80.0 \%$ & 31 & $53.4 \%$ & 151 & $72.6 \%$ \\
No & 26 & $17.3 \%$ & 23 & $39.7 \%$ & 49 & $23.6 \%$ \\
Prefer not to respond & 4 & $2.7 \%$ & 4 & $6.9 \%$ & 8 & $3.8 \%$
\end{tabular}

\begin{tabular}{lcccccc} 
& \multicolumn{2}{c}{ VACCINATED } & \multicolumn{2}{c}{ UNVACCINATED } & \multicolumn{2}{c}{ TOTAL SAMPLE } \\
\cline { 2 - 6 } Penis-Vagina Intercourse & $\boldsymbol{N}$ & Percentage & $\boldsymbol{N}$ & Percentage & $\boldsymbol{N}$ & Percentage \\
\hline Yes & 117 & $94.4 \%$ & 32 & $91.4 \%$ & 149 & $93.7 \%$ \\
No & 2 & $1.6 \%$ & 1 & $2.9 \%$ & 3 & $1.9 \%$ \\
Prefer not to respond & 5 & $4.0 \%$ & 2 & $5.7 \%$ & 7 & $4.4 \%$
\end{tabular}

\begin{tabular}{lcccccc} 
& \multicolumn{2}{c}{ VACCINATED } & \multicolumn{2}{c}{ UNVACCINATED } & \multicolumn{2}{c}{ TOTAL SAMPLE } \\
\cline { 2 - 7 } Penis-Anal Intercourse & $\boldsymbol{N}$ & Percentage & $\boldsymbol{N}$ & Percentage & $\boldsymbol{N}$ & Percentage \\
\hline Yes & 27 & $21.8 \%$ & 3 & $8.6 \%$ & 30 & $18.9 \%$ \\
No & 93 & $75.0 \%$ & 31 & $88.6 \%$ & 124 & $78.0 \%$ \\
Prefer not to respond & 4 & $3.2 \%$ & 1 & $2.9 \%$ & 5 & $3.1 \%$
\end{tabular}

Condom Use with New

Partner for Penis-Vagina

Intercourse

Always

Usually

Sometimes

Never

VACCINATED UNVACCINATED TOTAL SAMPLE

\begin{tabular}{cccccc}
$N$ & Percentage & $N$ & Percentage & $\boldsymbol{N}$ & Percentage \\
\hline 63 & $54.3 \%$ & 25 & $78.1 \%$ & 88 & $59.5 \%$ \\
36 & $31.0 \%$ & 4 & $12.5 \%$ & 40 & $27.0 \%$ \\
12 & $10.3 \%$ & 1 & $3.1 \%$ & 13 & $8.8 \%$ \\
5 & $4.3 \%$ & 2 & $6.3 \%$ & 7 & $4.7 \%$
\end{tabular}


Table 2 continued. Sexual risk factors $(N=208)$

\begin{tabular}{lcccccc} 
& \multicolumn{2}{c}{ VACCINATED } & UNVACCINATED & TOTAL SAMPLE \\
\cline { 2 - 7 } $\begin{array}{l}\text { Condom Use in } \\
\text { Relationship for Penis- }\end{array}$ & & & & & & \\
Vagina Intercourse & $\boldsymbol{N}$ & Percentage & $\boldsymbol{N}$ & Percentage & $\boldsymbol{N}$ & Percentage \\
\hline Always & 20 & $17.9 \%$ & 8 & $25.0 \%$ & 28 & $19.4 \%$ \\
Usually & 30 & $26.8 \%$ & 9 & $28.1 \%$ & 39 & $27.1 \%$ \\
Sometimes & 27 & $24.1 \%$ & 9 & $28.1 \%$ & 36 & $25.0 \%$ \\
Never & 35 & $31.3 \%$ & 6 & $18.8 \%$ & 41 & $28.5 \%$ \\
& & & & & & \\
& VACCINATED & UNVACCINATED & TOTAL SAMPLE \\
\hline
\end{tabular}

Condom Use with New

Partner for Penis-Anal

\begin{tabular}{lcccccc} 
Intercourse & $\boldsymbol{N}$ & Percentage & $\boldsymbol{N}$ & Percentage & $\boldsymbol{N}$ & Percentage \\
\hline Always & 14 & $51.9 \%$ & 3 & $100 \%$ & 17 & $56.7 \%$ \\
Usually & 2 & $7.4 \%$ & 0 & $0 \%$ & 2 & $6.7 \%$ \\
Sometimes & 4 & $14.8 \%$ & 0 & $0 \%$ & 4 & $13.3 \%$ \\
Never & 7 & $25.9 \%$ & 0 & $0 \%$ & 7 & $23.3 \%$
\end{tabular}

\begin{tabular}{lcccccc} 
& \multicolumn{2}{c}{ VACCINATED } & UNVACCINATED & TOTAL SAMPLE \\
\cline { 2 - 7 } $\begin{array}{l}\text { Condom Use in } \\
\text { Relationship for Penis- }\end{array}$ & & & & & & \\
Anal Intercourse & $\boldsymbol{N}$ & Percentage & $\boldsymbol{N}$ & Percentage & $\boldsymbol{N}$ & Percentage \\
\hline Always & 8 & $29.6 \%$ & 2 & $66.7 \%$ & 10 & $33.3 \%$ \\
Usually & 1 & $3.7 \%$ & 0 & $0 \%$ & 1 & $3.3 \%$ \\
Sometimes & 3 & $11.1 \%$ & 0 & $0 \%$ & 3 & $10.0 \%$ \\
Never & 15 & $55.6 \%$ & 1 & $33.3 \%$ & 16 & $53.3 \%$
\end{tabular}


Table 2 continued. Sexual risk factors $(\mathrm{N}=208)$

\begin{tabular}{lcccccc} 
& \multicolumn{2}{c}{ VACCINATED } & \multicolumn{2}{c}{ UNVACCINATED } & \multicolumn{2}{c}{ TOTAL SAMPLE } \\
\cline { 2 - 6 } Birth Control & $\boldsymbol{N}$ & Percentage & $\boldsymbol{N}$ & Percentage & $\boldsymbol{N}$ & Percentage \\
\hline Condoms Only & 23 & $15.3 \%$ & 11 & $19.0 \%$ & 34 & $16.3 \%$ \\
Multiple Methods & 35 & $23.3 \%$ & 7 & $12.1 \%$ & 42 & $20.2 \%$ \\
(Condoms \& pills, etc.) & & & & & & \\
Birth Control Pill Only & 67 & $44.7 \%$ & 13 & $22.4 \%$ & 80 & $38.5 \%$ \\
Depo Provera/Nuva Ring & 9 & $6.0 \%$ & 2 & $3.4 \%$ & 11 & $5.3 \%$ \\
Abstinence & 3 & $2.0 \%$ & 7 & $12.1 \%$ & 10 & $4.8 \%$ \\
Pull-Out Method & 1 & $0.7 \%$ & 0 & $0 \%$ & 1 & $0.5 \%$ \\
None & 3 & $2.0 \%$ & 6 & $10.3 \%$ & 9 & $4.3 \%$ \\
Prefer not to respond & 1 & $0.7 \%$ & 0 & $0 \%$ & 1 & $0.5 \%$ \\
N/A & 3 & $2.0 \%$ & 7 & $12.1 \%$ & 10 & $4.8 \%$ \\
No Answer & 5 & $3.3 \%$ & 5 & $8.6 \%$ & 10 & $4.8 \%$
\end{tabular}

\begin{tabular}{lcccccc} 
& \multicolumn{2}{c}{ VACCINATED } & \multicolumn{2}{c}{ UNVACCINATED } & \multicolumn{2}{c}{ TOTAL SAMPLE } \\
\cline { 2 - 7 } Unintended Pregnancy & $\boldsymbol{N}$ & Percentage & $\boldsymbol{N}$ & Percentage & $\boldsymbol{N}$ & Percentage \\
\hline Yes & 6 & $4 \%$ & 4 & $6.9 \%$ & 10 & $4.8 \%$ \\
No & 144 & $96 \%$ & 54 & $93.1 \%$ & 198 & $95.2 \%$
\end{tabular}

\begin{tabular}{lcccccc} 
& \multicolumn{2}{c}{ VACCINATED } & \multicolumn{2}{c}{ UNVACCINATED } & \multicolumn{2}{c}{ TOTAL SAMPLE } \\
\cline { 2 - 7 } Diagnosed with HPV & $\boldsymbol{N}$ & Percentage & $\boldsymbol{N}$ & Percentage & $\boldsymbol{N}$ & Percentage \\
\hline Yes & 16 & $10.7 \%$ & 3 & $5.2 \%$ & 19 & $9.1 \%$ \\
No & 132 & $88.0 \%$ & 54 & $93.1 \%$ & 186 & $89.4 \%$ \\
No Answer & 2 & $1.3 \%$ & 1 & $1.7 \%$ & 3 & $1.4 \%$
\end{tabular}


Table 2 continued. Sexual risk factors $(\mathrm{N}=208)$

\begin{tabular}{lcccccc} 
& \multicolumn{2}{c}{ VACCINATED } & \multicolumn{2}{c}{ UNVACCINATED } & \multicolumn{2}{c}{ TOTAL SAMPLE } \\
\cline { 2 - 7 } Abnormal Pap Smear & $\boldsymbol{N}$ & Percentage & $\boldsymbol{N}$ & Percentage & $\boldsymbol{N}$ & Percentage \\
\hline Yes & 31 & $20.7 \%$ & 11 & $19.0 \%$ & 42 & $20.2 \%$ \\
No & 118 & $78.7 \%$ & 47 & $81.0 \%$ & 165 & $79.3 \%$ \\
No Answer & 1 & $0.7 \%$ & 0 & $0 \%$ & 1 & $0.5 \%$
\end{tabular}

\begin{tabular}{lcccccc} 
& \multicolumn{2}{c}{ VACCINATED } & \multicolumn{2}{c}{ UNVACCINATED } & \multicolumn{2}{c}{ TOTAL SAMPLE } \\
\cline { 2 - 7 } $\begin{array}{l}\text { Atypical Cervical Cells or } \\
\text { Cervical Cancer }\end{array}$ & $\boldsymbol{N}$ & Percentage & $\boldsymbol{N}$ & Percentage & $\boldsymbol{N}$ & Percentage \\
\hline Yes & 12 & $8.0 \%$ & 3 & $5.2 \%$ & 15 & $7.2 \%$ \\
No & 137 & $91.3 \%$ & 54 & $93.1 \%$ & 191 & $91.8 \%$ \\
No Answer & 1 & $0.7 \%$ & 1 & $1.7 \%$ & 2 & $1.0 \%$
\end{tabular}

\begin{tabular}{lcccccc} 
& \multicolumn{2}{c}{ VACCINATED } & \multicolumn{2}{c}{ UNVACCINATED } & \multicolumn{2}{c}{ TOTAL SAMPLE } \\
\cline { 2 - 6 } Tested for STDs & $\boldsymbol{N}$ & Percentage & $\boldsymbol{N}$ & Percentage & $\boldsymbol{N}$ & Percentage \\
\hline Yes & 96 & $64.0 \%$ & 27 & $46.6 \%$ & 123 & $59.1 \%$ \\
No & 51 & $34.0 \%$ & 31 & $53.4 \%$ & 82 & $39.4 \%$ \\
No Answer & 3 & $2.0 \%$ & 0 & $0 \%$ & 3 & $1.4 \%$
\end{tabular}

\begin{tabular}{lcccccc} 
& \multicolumn{2}{c}{ VACCINATED } & \multicolumn{2}{c}{ UNVACCINATED } & \multicolumn{2}{c}{ TOTAL SAMPLE } \\
\cline { 2 - 7 } STD Diagnosis & $\boldsymbol{N}$ & Percentage & $\boldsymbol{N}$ & Percentage & $\boldsymbol{N}$ & Percentage \\
\hline Yes & 15 & $10 \%$ & 3 & $5.2 \%$ & 18 & $8.7 \%$ \\
No & 134 & $89.3 \%$ & 55 & $94.8 \%$ & 189 & $90.9 \%$ \\
Prefer not to respond & 1 & $7 \%$ & 0 & $0 \%$ & 1 & $0.5 \%$ \\
\hline
\end{tabular}


Table 3. Means, standard deviations, and internal consistencies for measures used with Vaccinated Sample

\begin{tabular}{lllllll}
\hline Measure & $\begin{array}{l}\text { Possible } \\
\text { Range }\end{array}$ & $\begin{array}{l}\text { Sample } \\
\text { Range }\end{array}$ & Scoring & Mean & SD & Alpha \\
\hline $\begin{array}{l}\text { Perceived } \\
\text { Susceptibility }\end{array}$ & $0-100 \%$ & $0-100 \%$ & $\begin{array}{l}10 \% \text { increment scale (higher= higher } \\
\text { susceptibility) }\end{array}$ & 46.52 & 20.87 & 0.92 \\
Perceived Severity & $6-42$ & $14-42$ & $\begin{array}{l}\text { Likert range 1-7 (higher= higher } \\
\text { severity) }\end{array}$ & 33.57 & 6.08 & 0.73 \\
Perceived Benefits & $6-42$ & $11-42$ & $\begin{array}{l}\text { Likert range 1-7 (higher=higher } \\
\text { benefits) }\end{array}$ & 35.92 & 5.39 & 0.81 \\
Perceived Barriers & $3-21$ & $3-15$ & $\begin{array}{l}\text { Likert range 1-7 (higher= higher } \\
\text { barriers) }\end{array}$ & 4.54 & 2.32 & 0.54 \\
Self-Efficacy & $8-56$ & $24-56$ & $\begin{array}{l}\text { Likert range 1-7 (higher= higher self- } \\
\text { efficacy) }\end{array}$ & 52.59 & 5.40 & 0.86 \\
Subjective Norms & $6-42$ & $10-42$ & $\begin{array}{l}\text { Likert range 1-7 (higher=more positive } \\
\text { subjective norms) }\end{array}$ & 36.87 & 6.62 & 0.88 \\
Cues to Action & $11-44$ & $15.1-40.4$ & $\begin{array}{l}\text { Likert range 1-4 (higher=more cues to } \\
\text { action) }\end{array}$ & 27.51 & 4.16 & 0.66 \\
& & $4-9$ & Multiple Choice & 7.56 & 1.15 & 0.36
\end{tabular}


Table 4. Means, standard deviations, and internal consistencies for measures used with Unvaccinated Sample

\begin{tabular}{|c|c|c|c|c|c|c|}
\hline Measure & $\begin{array}{l}\text { Possible } \\
\text { Range }\end{array}$ & $\begin{array}{l}\text { Sample } \\
\text { Range }\end{array}$ & Scoring & Mean & $S D$ & Alpha \\
\hline $\begin{array}{l}\text { Perceived } \\
\text { Susceptibility }\end{array}$ & $0-100 \%$ & $0-76.7 \%$ & $\begin{array}{l}10 \% \text { increment scale (higher= higher } \\
\text { susceptibility) }\end{array}$ & 34.60 & 20.45 & 0.92 \\
\hline Perceived Severity & $6-42$ & $16-42$ & $\begin{array}{l}\text { Likert range 1-7 (higher= higher } \\
\text { severity) }\end{array}$ & 32.42 & 6.34 & 0.73 \\
\hline Perceived Benefits & $6-42$ & $17-42$ & $\begin{array}{l}\text { Likert range 1-7 (higher=higher } \\
\text { benefits) }\end{array}$ & 30.43 & 6.43 & 0.83 \\
\hline Perceived Barriers & $3-21$ & $3-20$ & $\begin{array}{l}\text { Likert range 1-7 (higher=higher } \\
\text { barriers) }\end{array}$ & 7.03 & 4.11 & 0.72 \\
\hline Self-Efficacy & $8-56$ & $17-56$ & $\begin{array}{l}\text { Likert range 1-7 (higher= higher self- } \\
\text { efficacy) }\end{array}$ & 42.35 & 9.52 & 0.90 \\
\hline Subjective Norms & $6-42$ & $9-42$ & $\begin{array}{l}\text { Likert range } 1-7 \text { (higher=more positive } \\
\text { subjective norms) }\end{array}$ & 28.06 & 8.43 & 0.89 \\
\hline Cues to Action & $11-44$ & $15.0-36.6$ & $\begin{array}{l}\text { Likert range } 1-4 \text { (higher=more cues to } \\
\text { action) }\end{array}$ & 23.17 & 4.13 & 0.59 \\
\hline HPV Knowledge & $0-9$ & $4-9$ & $\mathrm{~T} / \mathrm{F}$ and Multiple Choice & 6.96 & 1.26 & 0.29 \\
\hline Vaccine Intentions & $4-28$ & $4-28$ & $\begin{array}{l}\text { Likert range } 1-7 \text { (higher=higher } \\
\text { intentions to receive the vaccine) }\end{array}$ & 11.91 & 6.69 & 0.88 \\
\hline
\end{tabular}




\section{Preliminary Correlations}

A correlation matrix of Pearson's r correlation coefficients was created to capture information about the relationships among all dichotomous and interval variables, including the demographic variables such as age, whether participants were sexually active, and how often participants had seen a gynecologist in the past two years. Due to the large number of correlations run, a more strict alpha value $(p<.01)$ was used to control for family-wise error. This information can be found in Figures 1, 2 and 3. Demographic and predictor variables that correlated with the four HBM variables as well as self-efficacy at medium to large effect $(r \geq .30)$ were controlled for in subsequent analyses (highlighted in Figures 1, 2 and 3). For vaccinated women, correlations between subjective norms and benefits ( $r=0.33$, medium effect) and between subjective norms and self-efficacy ( $r=0.31$, medium effect) were significant at the $p<.01$ level, such that higher perceived benefits and higher self-efficacy were related to higher levels of subjective norms for vaccinated women. The correlation between subjective norms and benefits ( $r=.39$, medium effect) also was significant for unvaccinated women. In addition, subjective norms and vaccination intentions ( $r=0.36$, medium effect) were correlated significantly as were barriers with HPV knowledge and sexual activity ( $r=-$ .036 for both, medium effects). Cues and self-efficacy ( $r=0.40$, medium effect) were also significantly correlated for unvaccinated women. When the correlations were run on the total sample, benefits and subjective norms ( $r=0.47$, medium effect) as well as selfefficacy and cues ( $r=0.46$, medium effect) continued to have significant correlations. Moreover, subjective norms and cues had significant correlations with vaccination status (respectively $r=.49$ and $r=.43$, medium effects) and with barriers (respectively $r=-.36$ and 
$r=-.34$, medium effects). Finally, self-efficacy correlated significantly with subjective norms ( $r=.49$, medium effect) and HPV knowledge ( $r=.31$, medium effect).

Sexual activity, HPV knowledge, cues and subjective norms were controlled for in regression analyses conducted only on unvaccinated women to account for the effects of these variables. HPV knowledge, cues and subjective norms were controlled for in regression analyses that compared vaccinated and unvaccinated women. To assess the relationship between the multi-level nominal demographic variables of ethnicity, relationship status and student status (first year, second year, third year, fourth year, fifth year, college graduate, graduate student) with the HBM and self-efficacy predictors and outcome variables, one-way ANOVAs were run using an alpha of 0.01 , finding no significant differences. Further analyses were computed to assess each of the research hypotheses and research questions. 
Figure 1. Correlations for Vaccinated Women

\begin{tabular}{|c|c|c|c|c|c|c|c|c|c|c|c|}
\hline & Susc & $\mathrm{Sev}$ & Ben & Barrier & $\mathrm{SE}$ & Norm & Cues & Know & Age & Sex & Gyn \\
\hline Susc & 1.00 & & & & & & & & & & \\
\hline Sev & .04 & 1.00 & & & & & & & & & \\
\hline Ben & .16 & $.32 * *$ & 1.00 & & & & & & & & \\
\hline Barrier & .03 & $.21 *$ & -.13 & 1.00 & & & & & & & \\
\hline SE & .12 & .08 & $.52 * *$ & $-.31 * *$ & 1.00 & & & & & & \\
\hline Norm & .04 & .15 & $.33 * *$ & -.15 & $.31^{* *}$ & 1.00 & & & & & \\
\hline Cues & .10 & -.09 & .14 & $-.18 *$ & $.21^{*}$ & .06 & 1.00 & & & & \\
\hline Know & -.11 & -.10 & -.04 & -.11 & $.21 * *$ & -.09 & $.17 *$ & 1.00 & & & \\
\hline Age & .02 & -.16 & .01 & -.03 & .14 & -.00 & -.03 & -.10 & 1.00 & & \\
\hline Sex & .01 & -.03 & .02 & -.03 & -.01 & .03 & .05 & .04 & .11 & 1.00 & \\
\hline Gyn & .12 & -.08 & -.04 & .04 & .08 & -.07 & $.20^{*}$ & .10 & $.16^{*}$ & $.26^{* *}$ & 1.00 \\
\hline
\end{tabular}

Key to Abbreviations in Figure 1: Susc (Perceived Susceptibility); Sev (Perceived Severity); Ben (Perceived Benefits); Barrier (Perceived Barriers); SE (Self-Efficacy to receive the vaccine); Norm (Subjective Norms); Cues (Cues to Action); Know (HPV Knowledge); Age (Current Age); Sex (Currently Sexually Active, 1 is yes, 0 is no); Gyn (Number of Gynecologist Visits Over the Past Two Yearst). Correlations significant at $\mathrm{p}<.01$ are marked with an “**” and correlations significant at $\mathrm{p}<.05$ are marked with an "*." 
Figure 2. Correlations for Non-Vaccinated Women

\begin{tabular}{|c|c|c|c|c|c|c|c|c|c|c|c|c|}
\hline & Intent & Susc & Sev & Ben & Barrier & $\mathrm{SE}$ & Norm & Cues & Know & Age & Sex & Gyn \\
\hline Intent & 1.00 & & & & & & & & & & & \\
\hline Susc & .05 & 1.00 & & & & & & & & & & \\
\hline Sev & .22 & .14 & 1.00 & & & & & & & & & \\
\hline Ben & $.46^{* *}$ & .24 & $.29 *$ & 1.00 & & & & & & & & \\
\hline Barrier & .12 & -.05 & -.13 & -.01 & 1.00 & & & & & & & \\
\hline SE & .12 & .23 & $.32 *$ & .23 & $-.56 * *$ & 1.00 & & & & & & \\
\hline Norm & $.36^{* *}$ & .13 & .21 & $.39 * *$ & $-.33 *$ & $.28^{*}$ & 1.00 & & & & & \\
\hline Cues & -.12 & .23 & .04 & -.01 & $-.33 *$ & $.40 * *$ & .02 & 1.00 & & & & \\
\hline Know & .07 & .12 & $.29 *$ & $.31 *$ & $-.36^{* *}$ & .24 & .25 & $.26^{*}$ & 1.00 & & & \\
\hline Age & .15 & -.14 & -.08 & $.28^{*}$ & -.04 & -.15 & .01 & -.07 & .01 & 1.00 & & \\
\hline Sex & .09 & .19 & -.03 & .01 & $-.36^{* *}$ & .19 & .18 & .16 & .16 & .12 & 1.00 & \\
\hline Gyn & .07 & .09 & .01 & .17 & $-.30 *$ & .20 & .19 & $.28 *$ & $.37 * *$ & .17 & $.35^{*}$ & 1.00 \\
\hline
\end{tabular}

Key to Abbreviations in Figure 2: Intent (Vaccination Intentions); Susc (Perceived Susceptibility); Sev (Perceived Severity); Ben (Perceived Benefits); Barrier (Perceived Barriers); SE (Self-Efficacy to receive the vaccine); Norm (Subjective Norms); Cues (Cues to Action); Know (HPV Knowledge); Age (Current Age); Sex (Currently Sexually Active, 1 is yes, 0 is no); Gyn (Number of Gynecologist Visits Over the Past Two Yearst). Correlations significant at $\mathrm{p}<.01$ are marked with an "**” and correlations significant at $\mathrm{p}<.05$ are marked with an "*." 
Figure 3. Correlations for Total Sample

\begin{tabular}{|c|c|c|c|c|c|c|c|c|c|c|c|c|}
\hline & Status & Susc & Sev & Ben & Barrier & $\mathrm{SE}$ & Norm & Cues & Know & Age & Sex & Gyn \\
\hline Status & 1.00 & & & & & & & & & & & \\
\hline Susc & $.25^{* *}$ & 1.00 & & & & & & & & & & \\
\hline $\mathrm{Sev}$ & .08 & .09 & 1.00 & & & & & & & & & \\
\hline Ben & $.40 * *$ & $.26 * *$ & $.32 * *$ & 1.00 & & & & & & & & \\
\hline Barrier & $-.36 * *$ & -.09 & .03 & $-.21 * *$ & 1.00 & & & & & & & \\
\hline SE & $.60 * *$ & $.27 * *$ & $.19 * *$ & $.52 * *$ & $-.55^{* *}$ & 1.00 & & & & & & \\
\hline Norm & $.49 * *$ & $.18^{*}$ & $.19 * *$ & $.47 * *$ & $-.36 * *$ & $.49 * *$ & 1.00 & & & & & \\
\hline Cues & $.43 * *$ & $.22 * *$ & -.01 & $.25 * *$ & $-.34 * *$ & $.46^{* *}$ & $.25^{* *}$ & 1.00 & & & & \\
\hline Know & $.22 * *$ & .01 & .04 & $.16^{*}$ & $-.27 * *$ & $.31 * *$ & .13 & $.26^{* *}$ & 1.00 & & & \\
\hline Age & -.09 & -.05 & $-.14^{*}$ & .11 & -.002 & -.03 & -.04 & -.07 & -.08 & 1.00 & & \\
\hline Sex & $.26^{* *}$ & .13 & -.01 & .11 & $-.25 * *$ & $.21 * *$ & $.20 * *$ & $.17 *$ & .12 & .09 & 1.00 & \\
\hline Gyn & $.22 * *$ & $.16^{*}$ & -.03 & .12 & $-.17 *$ & $.23 * *$ & .12 & $.29 * *$ & $.22 * *$ & $.14^{*}$ & $.32 * *$ & 1.00 \\
\hline
\end{tabular}

Key to Abbreviations in Figure 3: Status (Vaccination Status, 1 is vaccinated, 0 is non-vaccinated); Susc (Perceived Susceptibility); Sev (Perceived Severity); Ben (Perceived Benefits); Barrier (Perceived Barriers); SE (Self-Efficacy to receive the vaccine); Norm (Subjective Norms); Cues (Cues to Action); Know (HPV Knowledge); Age (Current Age); Sex (Currently Sexually Active, 1 is yes, 0 is no); Gyn (Number of Gynecologist Visits Over the Past Two Yearst). Correlations significant at $\mathrm{p}<.01$ are marked with an "**” and correlations significant at $\mathrm{p}<.05$ are marked with an "*." 


\section{Exploratory Factor Analysis for the HBM Variables}

Before the hypotheses could be tested, the HBM variables needed to be derived. An exploratory factor analysis (EFA) with a factor-extraction method of principle-axis factoring and oblimin rotation was conducted on each of the HBM variables (perceived susceptibility, severity, benefits and barriers). EFA was used to develop psychometrically sound subscales since the present study did not test pre-existing subscales representing each HBM variable. The items for the scale were developed based on previous HPV measures, HBM theory, and review by two experts in the field of HPV vaccination research and who were familiar with the HBM. Valid and reliable HBM subscales are lacking within the literature, especially for HPV vaccination. Moreover, most studies that use the HBM as a framework fail to include all four model variables. Since the sample in the current study included both vaccinated and unvaccinated women, it was important to retain items that had adequate psychometric qualities for the entire sample as well as each of these two subsamples. Therefore, EFAs for each subscale were conducted on the whole sample as well as on the vaccinated and unvaccinated subgroups. In order to retain as many items as possible, a structure coefficient of .30 was used as minimum criteria (Kahn, 2006). This means that items were retained if they had a structure coefficient of at least .30 for the entire sample as well as the two subsamples. In addition, it was determined that items would be dropped if they had a loading of .30 or above on more than one factor or if there was a difference of less than .20 between the primary and the cross-loading factor (Fabringar, Wegener, MacCallum, \& Strahan, 1999).

EFAs with one and two factors, where each factor represents a similar dimension within a construct, were conducted for each subscale in order to decide how many factors 
to retain. Empirical decisions of how many factors to retain were based on multiple criteria (Fabrigar et al., 1999; Tinsley \& Tinsley, 1987). For instance, Kahn's (2006) guidelines were followed which state that the number of factors to retain should be based on the percentage of variance among variables explained by each factor. Factors that explained a large percentage of variance to be deemed important were retained, and those that explained only a small percentage of variance were discarded (Kahn, 2006). In addition, this study followed Cattell's (1966) suggestion of looking at the scree plot to find the point where the eigenvalues flatten out. Factors were retained if they were above the last substantial drop in eigenvalues. According to the literature, there should be at least three to five items for each factor since EFAs produce more accurate results when factors are overdetermined (MacCallum, Widaman, Zhang \& Hong, 1999). Therefore, additional factors were dropped if they had less than three items.

Given the criteria for factor retention, a one factor solution was deemed the most appropriate for the susceptibility subscale since it contained six items and none of the items loaded onto a second factor. The subscale of severity contained six items and only two items loaded onto the second factor. Therefore a one factor solution was retained for the severity subscale. While the benefits subscale contained nine items, only one item loaded onto a second factor for the whole sample as well as for the vaccinated and unvaccinated subgroups. Thus, a one factor solution was retained for the benefits subscale. In addition, a one factor solution was deemed the most appropriate for the barriers subscale since it contained eight items and only two items loaded onto a second factor. All of the subscales, except for barriers, met the additional criterion for retaining factors. For instance, a one factor solution explained at least $40 \%$ of the variance and 
remained the only factor above the last substantial drop in eigenvalues (Appendices OQ). The barriers subscale explained $27.6 \%$ of the variance and appeared to have two or three factors above the last drop in eigenvalues (Appendix R). However, there were not enough items to justify including more than one factor since at least three items needed to be associated with each factor. This will be discussed further in the discussion chapter.

All six items for the susceptibility and severity subscales were retained since they met the structure coefficient criteria of .30 or above for the entire sample as well as the two subsamples (Tables 5 and 6). Six out of the nine items for the benefits subscale were retained as well. Initially, only two of the benefits items had a structure coefficient below .30. However, removing these items caused a third item to have a structure coefficient below .30 for vaccinated participants. Therefore, all three items were removed and a total of six items were retained. Tables 7, 8 and 9 show this progression. Three out of the eight items on the barriers subscale were retained (Tables 10 and 11), all of which referred to the stigma of receiving the HPV vaccine (ie. embarrassment) as opposed to logistical concerns (ie. expense, time constraints). This may indicate that stigma is emerging as the main barrier for HPV vaccination, which will be discussed more fully in other chapters. 
Table 5. Structure coefficients for perceived susceptibility

\begin{tabular}{|c|c|c|c|}
\hline & $\begin{array}{c}\text { Vaccinated } \\
(\mathrm{N}=150)\end{array}$ & $\begin{array}{c}\text { Unvaccinate } \\
\mathrm{d} \\
(\mathrm{N}=58)\end{array}$ & $\begin{array}{c}\text { Total } \\
\text { Sample } \\
(\mathrm{N}=208)\end{array}$ \\
\hline $\begin{array}{l}\text { What are your chances of contracting genital warts if you } \\
\text { didn't get Gardasil compared to other college-aged } \\
\text { women? } \\
\text { What are your chances of developing cervical cancer if } \\
\text { you didn't get Gardasil compared to other college-aged } \\
\text { women? } \\
\text { What are your chances of contracting HPV if you didn't } \\
\text { get Gardasil compared to other college-aged women? } \\
\text { What are your chances of contracting genital warts if you } \\
\text { didn't get Gardasil? } \\
\text { What are your chances of developing cervical cancer if } \\
\text { you didn't get Gardasil? } \\
\text { What are your chances of contracting HPV if you didn't } \\
\text { get Gardasil? }\end{array}$ & .720 & .717 & .722 \\
\hline
\end{tabular}


Table 6. Structure coefficients for perceived severity

\begin{tabular}{|c|c|c|c|}
\hline & $\begin{array}{l}\text { Vaccinated } \\
(\mathrm{N}=150)\end{array}$ & $\begin{array}{c}\text { Unvaccinate } \\
\mathrm{d} \\
(\mathrm{N}=58)\end{array}$ & $\begin{array}{c}\text { Total } \\
\text { Sample } \\
(\mathrm{N}=208)\end{array}$ \\
\hline $\begin{array}{l}\text { If I contracted HPV, it could hinder my future } \\
\text { relationships. } \\
\text { If I contracted HPV, it could hinder my present } \\
\text { relationship. } \\
\text { If I found out I had contracted HPV, I would be } \\
\text { devastated. } \\
\text { I would be embarrassed to let others know that I have } \\
\text { HPV if I found out that I contracted the disease. } \\
\text { If I contracted HPV, it could harm my future health. } \\
\text { Getting HPV could hinder my ability to become pregnant. }\end{array}$ & $\begin{array}{l}.384 \\
.398\end{array}$ & $\begin{array}{r}.481 \\
.333\end{array}$ & $\begin{array}{l}.396 \\
.380\end{array}$ \\
\hline
\end{tabular}


Table 7. Structure coefficients for perceived benefits

\begin{tabular}{|c|c|c|c|}
\hline & $\begin{array}{l}\text { Vaccinated } \\
(\mathrm{N}=150)\end{array}$ & $\begin{array}{c}\text { Unvaccinate } \\
\mathrm{d} \\
(\mathrm{N}=58) \\
\end{array}$ & $\begin{array}{c}\text { Total } \\
\text { Sample } \\
(\mathrm{N}=208)\end{array}$ \\
\hline $\begin{array}{l}\text { Getting Gardasil is important for my health. } \\
\text { One of the benefits to getting Gardasil is peace of mind } \\
\text { about my health. } \\
\text { One of the benefits to getting Gardasil, is feeling that I've } \\
\text { done everything I can do to protect myself against HPV. } \\
\text { Gardasil is effective in preventing cervical cancer. } \\
\text { Gardasil is effective in preventing an HPV infection. } \\
\text { How likely is it that the benefits of Gardasil outweigh the } \\
\text { potential side effects? } \\
\text { Gardasil is effective in preventing genital warts. } \\
\text { Gardasil is effective in preventing the spread of HPV to } \\
\text { partners. }{ }^{*} \\
\text { Gardasil is effective in preventing some types of oral } \\
\text { cancer.* }\end{array}$ & $\begin{array}{ll} & .789 \\
.796 & \\
& .608\end{array}$ & $\begin{array}{l}.808 \\
.680 \\
.687 \\
.689 \\
.584 \\
.565\end{array}$ & $\begin{array}{l}.778 \\
.775 \\
.651 \\
.651 \\
.610 \\
.566\end{array}$ \\
\hline
\end{tabular}

*Items removed from final analysis 
Table 8. Structure coefficients for perceived benefits revised 1

\begin{tabular}{|l|r|r|r|}
\hline & $\begin{array}{c}\text { Vaccinated } \\
(\mathrm{N}=150)\end{array}$ & $\begin{array}{r}\text { Unvaccinated } \\
(\mathrm{N}=58)\end{array}$ & $\begin{array}{r}\text { Total } \\
\text { Sample } \\
(\mathrm{N}=208)\end{array}$ \\
\hline Getting Gardasil is important for my health. & .814 & .775 & .797 \\
One of the benefits to getting Gardasil is peace of mind & .816 & .704 \\
about my health. & .610 & .713 \\
One of the benefits to getting Gardasil, is feeling that I've & & .603 \\
done everything I can do to protect myself against HPV. & .575 & .689 \\
Gardasil is effective in preventing cervical cancer. & .522 & .606 \\
How likely is it that the benefits of Gardasil outweigh the & .285 & .551 \\
potential side effects? Gardasil is effective in preventing & & .394 & .636 \\
an HPV infection. & & & .593 \\
Gardasil is effective in preventing genital warts. & & \\
\end{tabular}

*Items removed from final analysis

Table 9. Structure coefficients for perceived benefits revised 2

\begin{tabular}{|l|r|r|r|}
\hline & $\begin{array}{c}\text { Vaccinated } \\
(\mathrm{N}=150)\end{array}$ & $\begin{array}{r}\text { Unvaccinated } \\
(\mathrm{N}=58)\end{array}$ & $\begin{array}{r}\text { Total } \\
\text { Sample } \\
(\mathrm{N}=208)\end{array}$ \\
\hline One of the benefits to getting Gardasil is peace of mind & .836 & .768 & .826 \\
about my health. & .840 \\
Getting Gardasil is important for my health. & .616 & .824 \\
One of the benefits to getting Gardasil, is feeling that I've & .740 & .824 \\
done everything I can do to protect myself against HPV. & .570 \\
Gardasil is effective in preventing cervical cancer. & .529 & .633 \\
How likely is it that the benefits of Gardasil outweigh the & & .521 & .597 \\
potential side effects? & & .541 & .547 \\
Gardasil is effective in preventing an HPV infection. & & & .555 \\
& & & \\
\hline
\end{tabular}


Table 10. Structure coefficients for perceived barriers

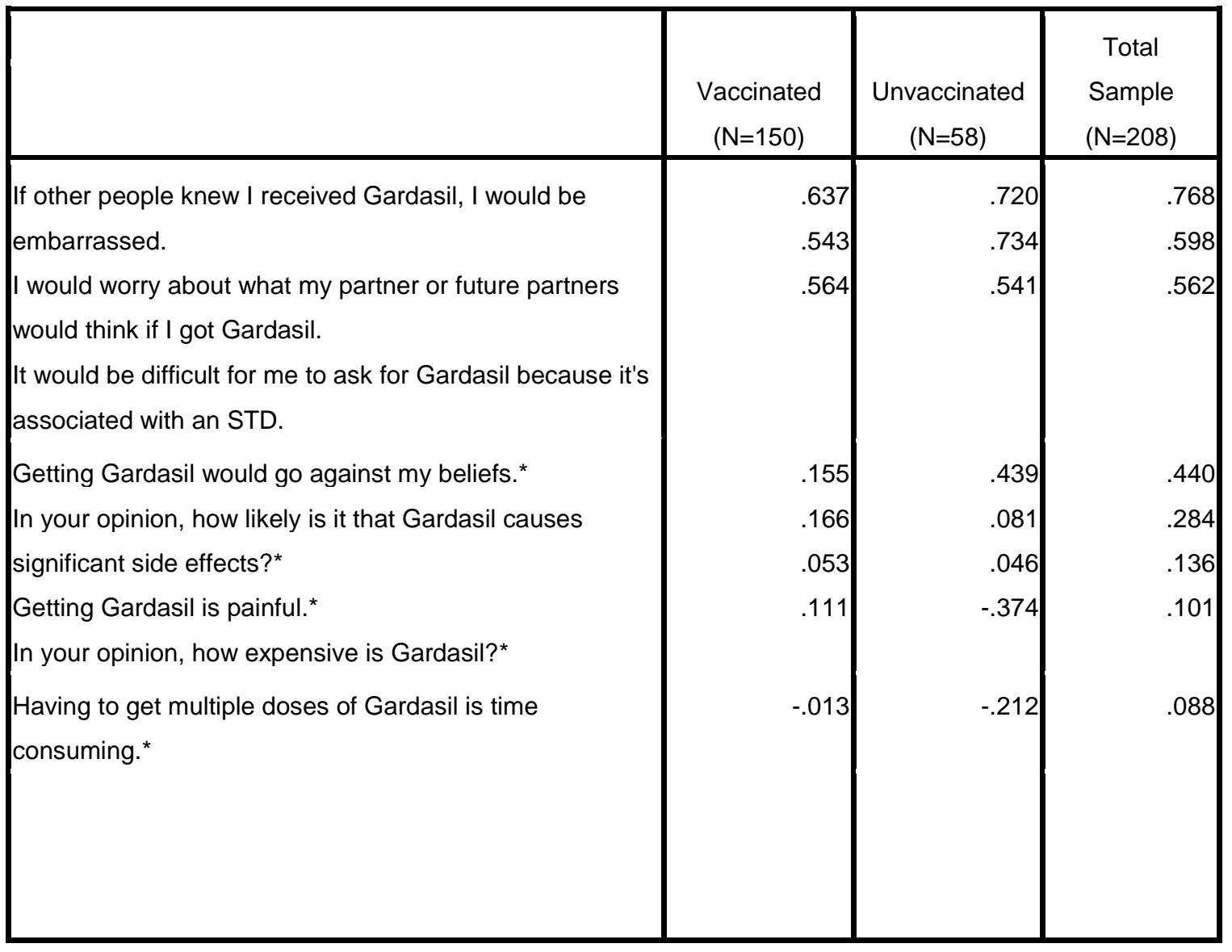

*Items removed from final analysis 
Table 11. Structure coefficients for perceived barriers revised

\begin{tabular}{|l|r|r|r|}
\hline & $\begin{array}{c}\text { Vaccinated } \\
(\mathrm{N}=150)\end{array}$ & $\begin{array}{c}\text { Unvaccinated } \\
(\mathrm{N}=58)\end{array}$ & $\begin{array}{r}\text { Total } \\
\text { Sample } \\
(\mathrm{N}=208)\end{array}$ \\
\hline If other people knew I received Gardasil, I would be & .628 & .895 \\
embarrassed. & .553 \\
I would worry about what my partner or future partners & .579 & .624 \\
would think if I got Gardasil. & & .562 \\
It would be difficult for me to ask for Gardasil because it's & & & .616 \\
associated with an STD. & & & \\
& & & \\
\end{tabular}

The main focus was on the HBM subscales since they were theorized to represent distinct constructs. However, an EFA was conducted on the items in their entirety to explore whether they loaded onto the expected subscales (Table 12). Each item was examined for sufficient loading on the hypothesized factor and for cross-loadings onto more than one factor. If an item had a loading of .30 or above on more than one factor or if there was a difference less than .20 between the primary and the cross-loading factor, then the item was not retained (Fabringar, Wegener, MacCallum, \& Strahan, 1999). All six items from the susceptibility subscale loaded cleanly onto Factor 2 and all six items from the severity subscale loaded cleanly onto Factor 3. Seven of the nine items from the benefits subscale loaded solely onto Factor 1, whereas one item double-loaded onto Factor 4 which represents barriers and one item loaded below .30. In addition, two items from the barriers subscale ("In your opinion, how likely is it that Gardasil causes significant side effects?" and "Getting Gardasil is painful.") loaded onto Factor 1 which 
represents benefits. It could be that participants did not view these aspects of getting the vaccine as large barriers, especially if they gave them low ratings. Finally, three of the eight items from the barriers subscale loaded solely onto Factor 4. Two items loaded below .30 and one item double-loaded onto another factor. Overall, $76 \%$ of the items on the HBM scale loaded significantly and solely onto the hypothesized subscales. These were also the same items retained after EFAs were performed on each subscale. Therefore, the final subscale items did not load significantly onto any other subscale. Most of the other $24 \%$ of the items loaded onto the hypothesized factors, but did not meet psychometric guidelines in that they did not load high enough or double loaded onto another factor. Only two items loaded onto a different construct than hypothesized which is likely the result of the wording of the items. The final items used in the HBM measure included those retained above as a result of the EFAs conducted on each of the HBM variables individually. 
Table 12. Structure coefficients for the HBM

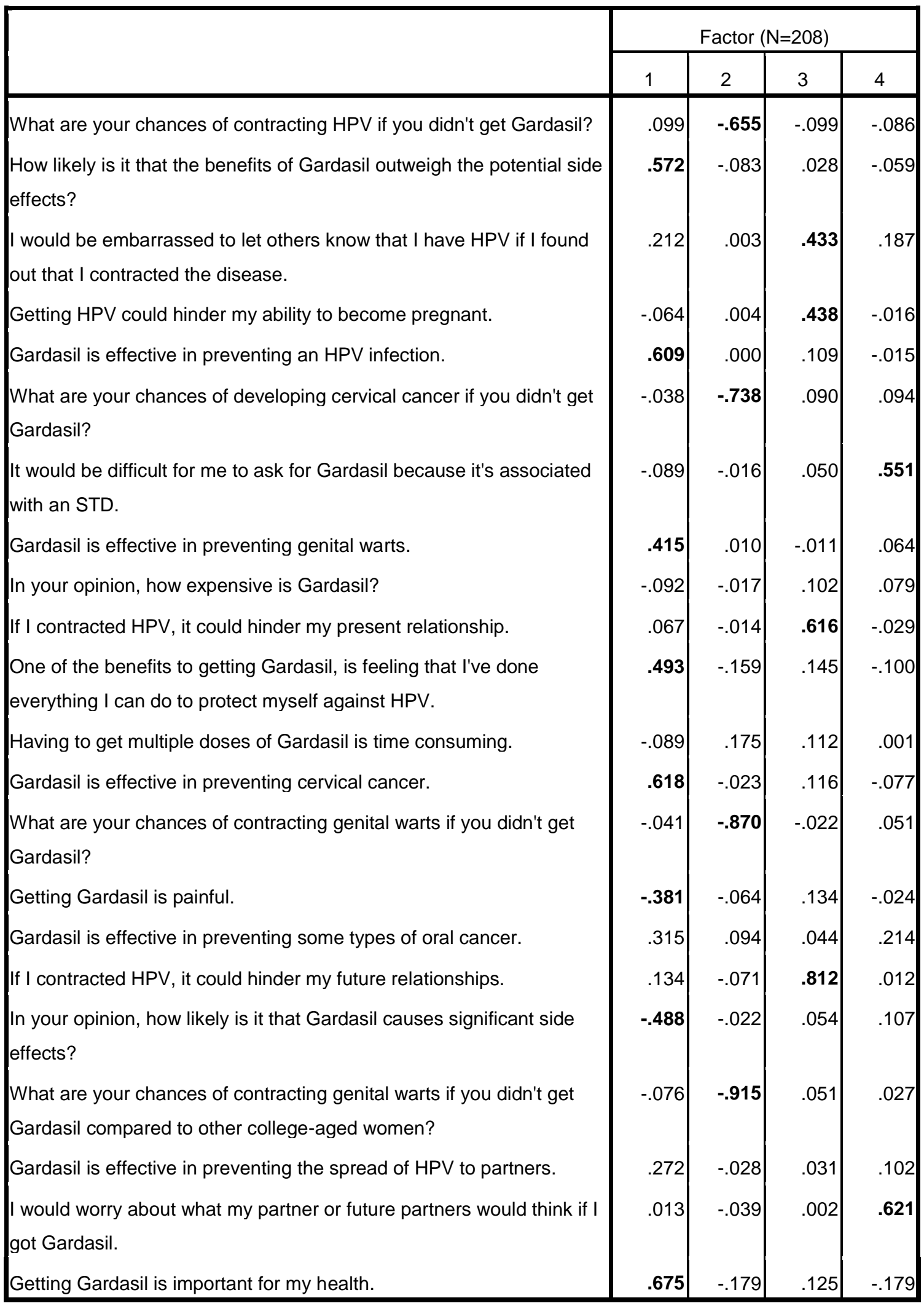


Table 12 continued. Structure coefficients for the HBM: benefits, barriers, susceptibility and severity

\begin{tabular}{|l|r|r|r|r|}
\hline & \multicolumn{3}{|c|}{ Factor (N=208) } \\
\cline { 2 - 5 } & \multicolumn{1}{|c|}{1} & \multicolumn{1}{c|}{2} & \multicolumn{1}{c|}{3} & \multicolumn{1}{c|}{4} \\
\hline What are your chances of contracting HPV if you didn't get Gardasil & -.004 & -.853 & -.015 & -.038 \\
compared to other college-aged women? & & & & \\
If other people knew I received Gardasil, I would be embarrassed. & .023 & .039 & -.162 & .826 \\
If I contracted HPV, it could harm my future health. & .006 & .049 & .435 & -.102 \\
One of the benefits to getting Gardasil is peace of mind about my & .676 & -.092 & .177 & -.116 \\
health. & & & & \\
What are your chances of developing cervical cancer if you didn't get & -.033 & -.879 & .087 & .008 \\
Gardasil compared to other college-aged women? & & & & \\
Getting Gardasil would go against my beliefs. & -.412 & .042 & -.045 & .309 \\
If I found out I had contracted HPV, I would be devastated. & .127 & -.006 & .554 & .007 \\
\hline
\end{tabular}

Factor $1=$ Benefits, Factor $2=$ Susceptibility, Factor $3=$ Severity, Factor $4=$ Barriers 


\section{Analysis of Hypotheses and Research Questions}

HPV Vaccination Intentions

Research Hypotheses

Hypothesis 1: HBM variables of perceived susceptibility, severity, benefits, barriers and self-efficacy will each contribute unique variance in predicting HPV vaccination intentions among unvaccinated women. Women who report greater perceived susceptibility to HPV, greater perceived severity of HPV, greater perceived benefits of HPV vaccination, fewer perceived barriers to HPV vaccination, and higher self-efficacy for receiving the HPV vaccine will report higher intentions to obtain the HPV vaccine.

Hierarchical regression analyses were run to assess vaccine intentions as the criterion variable after controlling for the variables of sexual activity, HPV knowledge, cues and subjective norms in the first step; susceptibility, severity, benefits, barriers and self-efficacy were included as the predictor variables in the second step (Table 13). The final multiple regression with both control and HBM variables included was statistically significant $\left(R^{2}=0.32, \mathrm{~F}_{5,43}=2.29, p<0.05\right.$, large effect $)$ and all nine variables accounted for $32 \%$ of the variance in vaccine intentions. However, the amount of additional variance predicted by the HBM variables of susceptibility, severity, benefits, barriers and self-efficacy after controlling for the variables of sexual activity, HPV knowledge, cues and subjective norms was not statistically significant $\left(\Delta R^{2}=0.17, \Delta \mathrm{F}_{5}\right.$, $\left.{ }_{43}=2.17, p>0.05\right)$. In the first model, subjective norms was a significant predictor of vaccine intentions $\left(t_{48}=2.45, p<0.05\right.$, medium effect $)$, however, in the final model, once the HBM variables were accounted for, subjective norms was no longer significant. In the 
final model, only benefits had a statistically significant effect on vaccine intentions $\left(t_{43}=2.13, p<0.05\right.$, medium effect $)$. In other words, for each additional point of perceived benefits, women's intentions for receiving the vaccine increased by 0.32 points, controlling for susceptibility, severity, barriers, self-efficacy, norms, cues, HPV knowledge and sexual activity. None of the control variables predicted unique variance after the HBM variables were included in the model.

Table 13: Summary of hierarchical regression predicting vaccine intentions

\begin{tabular}{|c|c|c|c|c|c|c|c|}
\hline $\begin{array}{l}\text { Predictors } \\
\text { partial } r^{2}\end{array}$ & $R$ & $\Delta R^{2}$ & $d f$ & $\Delta F$ & $\beta^{*}$ & $p$ & Semi- \\
\hline Step 1: & .39 & .15 & 48 & 2.17 & & .09 & \\
\hline Sexual Activity & & & & & 1.28 & .49 & .01 \\
\hline Cues & & & & & -.23 & .30 & .02 \\
\hline Subjective Norms & & & & & .26 & .02 & .11 \\
\hline HPV Knowledge & & & & & -.002 & .99 & .000 \\
\hline Step 2: & .57 & .17 & 43 & 2.17 & & .08 & \\
\hline Sexual Activity & & & & & 2.63 & .16 & .03 \\
\hline Cues & & & & & -.11 & .64 & .004 \\
\hline Subjective Norms & & & & & .21 & .07 & .05 \\
\hline HPV Knowledge & & & & & -.20 & .80 & .001 \\
\hline Susceptibility & & & & & -.03 & .49 & .01 \\
\hline Severity & & & & & .10 & .48 & .01 \\
\hline Benefits & & & & & .32 & .04 & .07 \\
\hline Barriers & & & & & .47 & .11 & .04 \\
\hline Self-Efficacy & & & & & .07 & .58 & .005 \\
\hline
\end{tabular}




\section{Hypothesis 2: There will be a positive correlation between HPV knowledge}

and HPV vaccination intentions for unvaccinated women

The correlation between HPV knowledge and HPV vaccination intentions for unvaccinated women was not significant $(r=0.07, p>0.05)$.

Hypothesis 3: There will be a positive relationship between reported HPV risk factors and HPV vaccination intentions for unvaccinated women.

Two t-tests for independent groups were conducted using sexually active (yes or no) and abnormal pap smear (yes or no) as the status variables and vaccination intentions as the outcome variable. Neither sexual activity $\left(t_{52}=-.64, p>.05\right)$ nor the occurrence of an abnormal pap smear $\left(t_{56}=-.40, p>.05\right)$ was significant. A Pearson correlation was conducted between vaccination intentions and number of sexual partners $(r=.13, p>.05)$ as well as between vaccination intentions and age of first intercourse $(r=-.10, p>.05)$. A Spearman correlation was conducted between vaccination intentions and frequency of condom use with a new partner $(\rho=.09, p>.05)$ and in a committed relationship $(\rho=.08$, $p>.05)$. None of these correlations were significant.

HPV Vaccination Status

Research Hypotheses

Hypothesis 4: The Health Belief Model (HBM) variables of perceived susceptibility, severity, benefits, barriers and self-efficacy will significantly distinguish vaccinated women from unvaccinated women. Specifically, vaccinated women will report greater perceived susceptibility to HPV, greater perceived severity of HPV, greater perceived benefits of HPV vaccination, fewer perceived 


\section{barriers to HPV vaccination, and greater self-efficacy for vaccination as compared to unvaccinated women.}

A logistic hierarchical regression analysis was run with HPV vaccination status as the dichotomous outcome variable after controlling for HPV knowledge, cues and subjective norms in the first step; perceived susceptibility, severity, benefits, barriers and self-efficacy were included the predictor variables in the second step (Table 14). The final logistic regression with both control and HBM variables included was statistically significant $\left(\chi_{8}^{2}=100.31, p<.001\right.$, large effect $)$. The addition of the HBM variables of susceptibility, severity, benefits, barriers and self-efficacy after controlling for the variables of sexual activity, HPV knowledge, cues and subjective norms was also statistically significant $\left(\Delta \chi^{2}=19.2, p<0.01\right.$, medium effect). After controlling for HPV knowledge, cues and subjective norms, self-efficacy (Wald $=9.05, p<0.01$ ) was the only significant HBM variable. This means that for a one point increase in the self-efficacy score, we expect the odds of being in the vaccinated group to become 1.118 times what they were (11.8\% increase in odds), holding all else constant. Cues (Wald=7.98, $p<0.01$ ) and subjective norms (Wald $=10.94, p<0.001)$ also remained significant after all of the variables were entered into the model. Therefore, a one point increase in the cues and subjective norms scores would increase the odds of being in the vaccinated group by $19.3 \%$ and $10.0 \%$ respectively. The Nagelkerke $R^{2}$, which is an approximation of the percent of variance explained, was 0.47 for the model with just the control variables and 0.56 when the HBM variables were included. 
Table 14: Summary of hierarchical logistical regression predicting vaccine status

\begin{tabular}{|c|c|c|c|c|c|c|}
\hline Predictors & $R^{2 *}$ & $\chi^{2}$ & $d f$ & Wald & $\operatorname{Exp}(B)$ & $p$ \\
\hline $\begin{array}{ll}\text { Step 1: } & \\
& \text { Cues } \\
& \text { Subjective Norms } \\
& \text { HPV Knowledge }\end{array}$ & .47 & 81.12 & 3 & $\begin{array}{r}22.05 \\
30.30 \\
1.31\end{array}$ & $\begin{array}{l}1.31 \\
1.15 \\
1.20\end{array}$ & $\begin{array}{l}.000 \\
.000 \\
.000 \\
.25\end{array}$ \\
\hline $\begin{array}{l}\text { Step 2: } \\
\text { Cues } \\
\text { Subjective Norms } \\
\text { HPV Knowledge } \\
\text { Susceptibility } \\
\text { Severity } \\
\text { Benefits } \\
\text { Barriers } \\
\\
\text { Self-Efficacy }\end{array}$ & .56 & 100.31 & 8 & $\begin{array}{r}7.98 \\
10.94 \\
.38 \\
.89 \\
1.45 \\
.89 \\
.19 \\
9.05\end{array}$ & $\begin{array}{r}1.19 \\
1.10 \\
1.12 \\
1.01 \\
.96 \\
1.04 \\
1.04 \\
1.12\end{array}$ & $\begin{array}{l}.000 \\
.005 \\
.001 \\
.54 \\
.34 \\
.23 \\
.35 \\
.66 \\
.003\end{array}$ \\
\hline
\end{tabular}

*Nagelkerke R Square

Hypothesis 5: Vaccinated women will report higher self-efficacy to receive the vaccine as compared to unvaccinated women.

A two sample t-test for independent groups was conducted using vaccination status as the status variable and self-efficacy as the outcome variable. The Levene's Test for equality of variances was significant $(\mathrm{F}=28.61, p<.001)$, therefore equal variances were not assumed. Vaccinated women reported significantly higher vaccination selfefficacy as compared to unvaccinated women $\left(t_{72}=-8.48, p<0.001\right.$, large effect $)$.

Hypothesis 6: Vaccinated women will report more positive cues to action for receiving the HPV vaccine as compared to unvaccinated women.

A two sample t-test for independent groups was conducted using vaccination status as the status variable and number of positive cues to action as the outcome 
variable. Vaccinated women reported significantly higher cues to action as compared to unvaccinated women $\left(t_{205}=-6.74, p<0.001\right.$, large effect $)$.

\section{Hypothesis 7: Vaccinated women will report higher subjective norms than}

\section{unvaccinated women.}

A two sample t-test for independent groups was conducted using vaccination status as the status variable and subjective norms as the outcome variable. The Levene's Test for equality of variances was significant $(\mathrm{F}=6.82, p<.05)$, therefore equal variances were not assumed. Vaccinated women reported significantly higher subjective norms for receiving the vaccine as compared to unvaccinated women $\left(t_{86}=-7.16, p<0.001\right.$, large effect).

\section{Hypothesis 8: Vaccinated women will have higher HPV knowledge scores}

\section{than unvaccinated women.}

A two sample t-test for independent groups was conducted using vaccination status as the status variable and HPV knowledge as the outcome variable. Vaccinated women had significantly higher levels of HPV knowledge than unvaccinated women $\left(t_{206}=-3.28, p<0.05\right.$, small-medium effect $)$.

\section{Hypothesis 9: Vaccinated women will report higher levels of HPV risk factors}

\section{than unvaccinated women.}

Four chi-square analyses were conducted between vaccination status and the four HPV risk factors of sexually active (yes or no), frequency of condom use with new partners and in a committed relationship (always, often, sometimes, never), and abnormal pap smear (yes or no). Significantly more vaccinated women reported being sexually

active $\left(\chi^{2}=13.09, p<0.001\right)$ than unvaccinated women. However, there was no significant 
difference in terms of condom use with new partners $\left(\chi^{2}=7.27, p>.05\right)$, condom use in a committed relationship $\left(\chi^{2}=2.20, p>.05\right)$, and abnormal pap smears $\left(\chi^{2}=0.09, p>.05\right)$. For condom use, both groups of women were also compared on the dimensions of always using a condom and significantly more unvaccinated women reported always using a condom with a new partner $\left(\chi^{2}=5.90, p<.05\right)$ as compared to vaccinated women. There was no significant difference between the groups for always using a condom in a committed relationship $\left(\chi^{2}=0.81, p>.05\right)$. Additionally, two t-tests with independent groups were conducted with vaccination status as the status variable and number of sexual partners and age of first intercourse as the outcome variables. Vaccinated women had a significantly higher number of sexual partners $\left(t_{70}=-2.01, p<.05\right.$, small effect $)$ as compared to unvaccinated women but there was no significant difference in age of first intercourse $\left(t_{145}=.022, p>.05\right)$.

Research Questions

\section{Research Question 1: What is the prevalence of vaccinated women in the}

\section{sample?}

In this sample, 150 women out of 208 had received at least one of the HPV vaccine shots, which results in a prevalence rate of $72 \%$. However, recruitment in this study was combined with another HPV study and unvaccinated participants were randomly assigned to either this study or the other HPV study. Therefore, 58 unvaccinated women participated in this study and 89 unvaccinated women participated in the other HPV study, providing a total of 147 . Therefore, the prevalence of vaccinated women when considering both studies was 50.5\%. Twenty-two participants had started 
but not completed the series which results in $43.1 \%$ of the sample who had completed the full vaccination series and $7.4 \%$ started, but had not completed the series.

Question 2: For vaccinated women, is there a significant correlation between the length of time since vaccination and the HBM components of perceived benefits, barriers, susceptibility, and severity?

The correlation between length of time since vaccination and the four HBM variables was not significant (benefits: $r=-0.08, p>0.05$; barriers: $r=-0.10, p>0.05$;

susceptibility: $r=\quad-0.03, p>0.05$; severity: $r=0.03, p>0.05)$.

Question 3: For vaccinated women, is there a significant correlation between satisfaction with receiving the HPV vaccine and the HBM components of perceived benefits, barriers, susceptibility, and severity?

The correlation was significant between the variables of satisfaction with receiving the HPV vaccine and perceived severity $(r=0.26, p<0.001$, small effect) as well as between satisfaction and perceived benefits ( $r=0.65, p<0.001$, large effect). However, the correlation was not significant between the variables of satisfaction and perceived susceptibility $(r=0.09, p>0.05)$ or between satisfaction and perceived barriers $(r=-0.15$, $p>0.05)$.

Question 4: Do women who report higher risk factors for HPV report higher perceived susceptibility to HPV?

Two t-tests for independent groups were conducted using sexually active (yes or no) and abnormal pap smear (yes or no) as the status variables and susceptibility as the outcome variable. Women who had an abnormal pap smear reported significantly higher perceived susceptibility $\left(t_{205}=2.09, p<.05\right.$, small effect $)$ than women who did not have an 
abnormal pap. However, there was no significant difference for sexual activity $\left(t_{198}=-\right.$ $1.80, p>.05)$. A Pearson correlation was conducted between susceptibility and number of sexual partners $(r=.18, p<.05$, small effect) as well as between susceptibility and age of first intercourse $(r=-.10, p>.05)$. A Spearman correlation was conducted between susceptibility and frequency of condom use with a new partner $(\rho=.06, p>.05)$ and in a committed relationship ( $\rho=.02, p>.05)$. The only significant correlation was between perceived susceptibility and number of sexual partners, meaning that the greater the number of sexual partners, the greater the perceived susceptibility to HPV.

\section{Analysis of Open-Ended Questions}

Question 5: How will women respond to the following open-ended questions: What do you view as the greatest benefits of HPV vaccination? What do you view as the greatest drawbacks of HPV vaccination?

A team of two raters first reviewed the responses to these questions to generate common themes or categories. One rater was a 60 -year-old white, female faculty member and the other rater was a 26 year-old white, female third-year doctoral student. The raters coded a subsample of the responses to see if these categories needed refinement. After further refinement of the categories, they rated the items to see if any other changes needed to be made in the categories. Then a team of three raters coded each response into the identified categories and could assign multiple categories to a response if deemed necessary. These raters included the 26-year-old female described above, a 27-year-old white, female third-year doctoral student and a 32-year-old white, female fourth-year doctoral student. Inter-rater reliability was determined by calculating kappas for each pair of raters. Since multiple categories could be assigned to a response, agreement was based 
on the individual categories. For instance, if rater A assigned categories 1 and 2 to a response and rater B assigned only category 1, it would be marked as agreement for category 1 and disagreement for category 2.

Responses to the open-ended question inquiring as to the benefits of HPV vaccination fit into the categories listed in Table 15. In addition, chi-squares were conducted to see if a significant difference existed between vaccinated and unvaccinated women for each category. For the chi-squares, a contingency table was created with four cells representing the observed number of vaccinated participants who had and had not described a category and the number of unvaccinated participants who had and had not described the category. The chi-square test involved comparing these observed values with the expected values. The majority of responses for both vaccinated and unvaccinated participants described the benefits of prevention (e.g., reduced risk of contracting HPV, gential warts or cervical cancer). There was no significant difference between these two groups of women for this category. However, a significantly greater proportion of vaccinated women described reducing worry or anxiety (e.g., peace of mind, feeling safe) as a benefit of receiving the vaccine as compared to unvaccinated women $\left(\chi^{2}=3.93\right.$, $p<.05)$. Although very few respondents indicated that they had no need or interest in the vaccine, a significantly greater proportion of unvaccinated women cited this response as compared to vaccinated women $\left(\chi^{2}=7.57, p<.01\right)$. Almost $20 \%(18.6 \%)$ of responses cited more than one category that was considered the best aspect of receiving the vaccine. Among the three coders, cohen's kappa values ranged from .76 to .82, with an average cohen's kappa value of .79 . 
Responses to the open-ended question inquiring as to the drawbacks of HPV vaccination fit into the categories listed in Table 16. The most common response from vaccinated women was that there was no drawback to getting the vaccine. On the other hand, the majority of unvaccinated women cited concerns about the efficacy and safety of the vaccine (e.g., unknown side-effects), as the biggest drawback to getting vaccinated. Although vaccinated participants also described concerns about efficacy of the vaccine, there was a greater proportion of unvaccinated participants who responded with this drawback. Both groups reported logistical concerns about getting the vaccine (e.g., cost, time-consuming) and having to get a shot (e.g., pain of shot) as drawbacks to vaccination. More unvaccinated women reported stigma and embarrassment, not perceiving self to be at risk, or no need or interest in the vaccine as drawbacks as compared to vaccinated women. Slightly over a quarter $(28.5 \%)$ of responses cited more than one category that was considered the worst aspect of receiving the vaccine. Among the three coders, cohen's kappa values ranged from .91 to .93, with an average cohen's kappa value of .92.

Chi-squares were conducted to see if a significant difference existed between vaccinated and unvaccinated women for each category. A significantly greater proportion of unvaccinated women reported concerns about safety $\left(\chi^{2}=13.83, p<.001\right)$, not perceiving themselves to be at risk $\left(\chi^{2}=19.29, p<.001\right)$, concern about stigma $\left(\chi^{2}=4.81\right.$, $p<.05)$, and no need or interest in the vaccine $\left(\chi^{2}=13.63, p<.001\right)$ as compared to vaccinated women. Conversely, a significantly greater proportion of vaccinated women reported concerns about having to get a shot $\left(\chi^{2}=4.05, p<.05\right)$ and no perceived drawbacks to vaccination $\left(\chi^{2}=18.25, p<.001\right)$ as compared to unvaccinated women. There 
was no significant difference between vaccinated and unvaccinated women reporting logistical concerns as a drawback. 
Table 15. Responses to the greatest benefits of HPV vaccination

\begin{tabular}{|c|c|c|c|c|c|c|c|c|c|}
\hline \multirow[b]{2}{*}{ Categories } & \multicolumn{2}{|c|}{$\begin{array}{c}\text { VACCINATED } \\
(N=141)\end{array}$} & \multicolumn{2}{|c|}{$\begin{array}{c}U N- \\
V A C C I N A T E D \\
(N=52)\end{array}$} & \multicolumn{2}{|c|}{$\begin{array}{c}\text { TOTAL } \\
\text { SAMPLE } \\
(N=193)\end{array}$} & \multirow[b]{2}{*}{$\chi^{2}$} & \multirow[b]{2}{*}{$d f$} & \multirow[b]{2}{*}{$p$} \\
\hline & Count & $\%$ & Count & $\%$ & Count & $\%$ & & & \\
\hline Prevention & 108 & $76.6 \%$ & 38 & $73.1 \%$ & 146 & $75.6 \%$ & 0.26 & 1 & $>.05$ \\
\hline Reduced worry & 60 & $42.6 \%$ & 14 & $26.9 \%$ & 74 & $38.3 \%$ & 3.93 & 1 & $<.05$ \\
\hline No need or interest & 3 & $2.1 \%$ & 6 & $11.5 \%$ & 9 & $4.7 \%$ & 7.57 & 1 & $<.01$ \\
\hline
\end{tabular}

*The total percentage exceeds $100 \%$ since some responses were placed into multiple categories.

Table 16. Responses to the greatest drawbacks of HPV vaccination

\begin{tabular}{|c|c|c|c|c|c|c|c|c|c|}
\hline \multirow[b]{2}{*}{ Categories } & \multicolumn{2}{|c|}{$\begin{array}{c}\text { VACCINATED } \\
(N=138)\end{array}$} & \multicolumn{2}{|c|}{$\begin{array}{c}\text { UN- } \\
\text { VACCINATED } \\
(N=52)\end{array}$} & \multicolumn{2}{|c|}{$\begin{array}{c}\text { TOTAL } \\
\text { SAMPLE } \\
(N=190)\end{array}$} & \multirow[b]{2}{*}{$\chi^{2}$} & \multirow[b]{2}{*}{$d f$} & \multirow[b]{2}{*}{$p$} \\
\hline & Count & $\%$ & Count & $\%$ & Count & $\%$ & & & \\
\hline Concerns about safety & 35 & $25.4 \%$ & 28 & $53.8 \%$ & 63 & $33.2 \%$ & 13.83 & 1 & $<.001$ \\
\hline No drawbacks & 55 & $39.9 \%$ & 4 & $7.7 \%$ & 59 & $31.1 \%$ & 18.25 & 1 & $<.001$ \\
\hline Logistical concerns & 41 & $29.7 \%$ & 14 & $26.9 \%$ & 55 & $28.9 \%$ & 0.14 & 1 & $>.05$ \\
\hline Having to get a shot & 41 & $29.7 \%$ & 8 & $15.4 \%$ & 49 & $25.8 \%$ & 4.05 & 1 & $<.05$ \\
\hline $\begin{array}{l}\text { Do not perceive self to } \\
\text { be at risk }\end{array}$ & 0 & $0.0 \%$ & 7 & $13.5 \%$ & 7 & $3.7 \%$ & 19.29 & 1 & $<.001$ \\
\hline Stigma & 2 & $1.4 \%$ & 4 & $7.7 \%$ & 6 & $3.2 \%$ & 4.81 & 1 & $<.05$ \\
\hline No need or interest & 0 & $0.0 \%$ & 5 & $9.6 \%$ & 5 & $2.6 \%$ & 13.63 & 1 & $<.001$ \\
\hline
\end{tabular}




\section{What factors led you to get vaccinated?}

\section{What factors keep you from getting vaccinated?}

These final two open-ended questions were analyzed through the same procedure as the benefits and drawbacks of vaccination open-ended questions. Only vaccinated participants responded to the question about what factors led them to get vaccinated. Responses to this open-ended question fit into the categories listed in Table 17. Responses indicated that the influence of a doctor or medical professional, the desire to be protected and the influence of their mother were the most common factors that led participants to receive the vaccine. Less common responses included perceiving themselves to be at risk, being influenced by a friend or sibling, being influenced by advertisements or the media, being influenced by their father, and expressing little or no downsides. A high number (81.4\%) of responses cited more than one category as a factor that led participants to get the vaccine. Among the three coders, cohen's kappa values ranged from .86 to .89 with an average cohen's kappa value of .88 .

Table 17. Responses to the factors that led to HPV vaccination

\begin{tabular}{lcc}
\hline & \multicolumn{2}{c}{ Vaccinated $(\boldsymbol{N}=\mathbf{1 4 0})$} \\
\cline { 2 - 3 } Categories & Count & Percentage \\
\hline Influenced by doctor & 68 & $48.6 \%$ \\
Wanting to protect self & 50 & $35.7 \%$ \\
Influenced by mother & 47 & $33.6 \%$ \\
Perceive self to be at risk & 22 & $15.7 \%$ \\
Influenced by friend or sibling & 21 & $15.0 \%$ \\
Little or no downsides & 17 & $12.1 \%$ \\
Influenced by advertisements or media & 16 & $11.4 \%$ \\
Influenced by father & 13 & $9.3 \%$ \\
\hline
\end{tabular}

*The total percentage exceeds $100 \%$ since some responses were placed into multiple categories. 
Only unvaccinated participants responded to the question about which factors kept them from getting vaccinated. Responses to this open-ended question fit into the categories listed in Table 18. An equal number of responses described concerns about the efficacy and safety of the vaccine and not perceiving themselves to be at risk as the biggest factors keeping participants from getting the vaccine. Almost a quarter of responses reported logistical concerns about getting the vaccine. Less common responses included having no need or interest in the vaccine, thinking that the vaccine won't help non-virgins and concerns about having to get a shot. Only one response cited stigma and embarrassment as a factor keeping the participant from becoming vaccinated. Slightly less than half $(41.4 \%)$ of responses cited more than one category as a factor that contributed to participants not getting the vaccine. Among the three coders, cohen's kappa values ranged from .81 to .94 with an average cohen's kappa value of .87 .

Table 18. Responses to the factors that keep participants from getting the HPV

\begin{tabular}{lcc}
\multicolumn{3}{c}{ vaccine } \\
\cline { 2 - 3 } Categories & Unvaccinated $(\mathbf{N = 5 3 )}$ \\
\hline Concerns about safety of vaccine & 22 & Percentage \\
Do not perceive self to be at risk & 22 & $41.5 \%$ \\
Logistical concerns & 13 & $41.5 \%$ \\
No need or interest in vaccine & 6 & $11.3 \%$ \\
Not effective for non-virgins & 6 & $11.3 \%$ \\
Having to get a shot & 5 & $9.4 \%$ \\
Stigma and embarrassment & 1 & $1.9 \%$ \\
\hline
\end{tabular}

*The total percentage exceeds $100 \%$ since some responses were placed into multiple categories. 


\section{Chapter 6}

\section{Discussion}

This chapter presents a summary and comparative description of women who had, and who had not, received a vaccine to prevent HPV. These two samples did not take exactly the same measures. For instance, unvaccinated women completed the HPV vaccination intentions measure, but vaccinated women did not. However, both samples completed the HBM measures as well as the self-efficacy, subjective norms, cues to action and HPV knowledge measures. Some hypotheses were specific for only unvaccinated women, only vaccinated women or for comparing the two samples. Very few studies have used the HBM to understand decision-making around the HPV vaccine, especially for college-aged women. Therefore, the current study fills a gap in the literature. A discussion of the results of the hypotheses and research questions are provided, beginning with unvaccinated women only, then comparing unvaccinated and vaccinated women, looking at vaccinated women only and ending with the open-ended questions. Then, a discussion of the study's limitations and suggested implications for future research and clinical practice are included. However, before examining the hypotheses or future implications, some description of the two groups of women comprising the sample is necessary.

\section{Overview of sample}

In this sample, approximately half $(50.5 \%)$ of the participants had received at least one of the HPV vaccine shots. This is higher than the 2008 national percentage of vaccinated adolescents (37\%) and percentages of vaccinated college women (12\%) from studies conducted in 2007 (Allen et al., 2009; CDC, 2009; Grant et al., 2009). The 2008 
national survey of vaccination coverage among adolescents found regional differences in the percentage of females who had received the HPV vaccine (CDC, 2009). However, according to the survey, the main states represented in the present study had vaccination coverage close to the national average (Maryland $=41 \%$ and D.C. $=39 \%$ ). Therefore, regional differences may not account for the higher levels of vaccination uptake in this study. Instead, it appears that HPV vaccination has increased the longer it has been available and that rates are fairly high among students who elected to participate in the current study.

Since the vaccine has only been available since 2006 , the women in this study were not able to get vaccinated during the recommended age range of 11-12. Therefore, the women in this sample, who were in their late teens and early 20 's, may have been especially motivated to receive the vaccine before they turned 26 and were no longer eligible. Moreover, women at this age are more likely to link the vaccine with becoming sexually active as compared to 11-12 year old girls. In the total sample, 22 participants (7.4\%) had started but not completed the series and among these women, 17 reported that they planned to become fully vaccinated. Therefore, the majority of women in this study who started the vaccine plan to complete it. This differs from national samples which report that more adolescents initiate the vaccine than complete the series. These findings suggest that college-age women may be more committed to completing the vaccine series than adolescents. While the prevalence of vaccinated women provides important information, the hypotheses and research questions related to unvaccinated women can help us better understand the factors that influence whether or not women get the vaccine. 


\section{Findings about Unvaccinated Women}

\section{Relationship of HBM to HPV vaccination intentions in unvaccinated women.}

One purpose of this study was to explore which HBM variables predict HPV vaccination intentions among participants who had not yet received the HPV vaccine. Vaccine intentions were defined as the extent that women intended to get the vaccine (i.e. I expect that I will get vaccinated against HPV within the next year) and ranged from low intentions to high intentions. Since the vaccine has been available for several years, it is important to determine which factors may influence women in their decision to receive or to not receive the vaccine. The variables of sexual activity, HPV knowledge, cues to action and subjective norms were controlled for before examining the HBM variables. When these variables were included in the analysis prior to the HBM variables, subjective norms was the only factor that predicted vaccine intentions. However, once the HBM variables were included, subjective norms was no longer significant. Cues to action, sexual activity and HPV knowledge were not significant predictors of vaccine intentions at any step in the analysis. Once the HBM variables were included, the findings showed that perceived benefits of receiving the vaccine significantly predicted HPV vaccine intentions. Perceived benefits remained a significant predictor of vaccine intentions even after controlling for the other HBM variables (barriers, susceptibility, severity and self-efficacy). Thus, perhaps the best determinant of vaccine intentions is how effective and beneficial women perceive the vaccine to be in protecting them against HPV and its consequences. This finding is supported by the literature which found an association between higher perceived vaccine effectiveness and greater HPV vaccine intentions (Brewer \& Fazekas, 2007). The current study used a broader definition of 
perceived benefits by including not only vaccine effectiveness, but also peace of mind about health and the feeling of doing everything possible to protect the self against HPV.

In contrast to what was hypothesized based on the previous literature, the rest of the HBM variables, including perceived susceptibility, severity, barriers and selfefficacy, did not significantly predict HPV vaccination intentions. The discrepancy in these findings may be due to several reasons. First, most previous studies on HPV vaccine intentions did not control for other important variables, like sexual activity, HPV knowledge, cues and subjective norms. Second, many previous studies on HPV vaccine intentions as well as other health decisions did not include all of the HBM variables in the same model (Boehner et al., 2003; Ramirez et al., 1997; Yacobi et al., 1999). Therefore, the analyses conducted in this study may represent more rigorous testing of the HBM model since confounding factors such as, sexual activity, HPV knowledge, cues to action and subjective norms, were controlled for and the entire HBM model was included. The HBM variables may not be as independent as originally thought and this overlap could have affected the results. For instance, perceived severity was significantly correlated with perceived benefits and self-efficacy, and perceived barriers was also significantly correlated with self-efficacy although these were small to moderate correlations. Finally, the HPV vaccine was not yet available at the time that many of the studies about HPV vaccine acceptance were conducted. The discrepancy in results may reflect the difference in the actual availability of the HPV vaccine. Different factors may become more salient when a vaccine has been available for several years in contrast to when a vaccine is represented as a hypothetical possibility. 


\section{Relationship between HPV knowledge and vaccination intentions in}

unvaccinated women. The relationship between HPV knowledge and HPV vaccination intentions was not significant among participants who had not received the vaccine. It is possible that since the vaccine has been available for three years and there have been many advertisements in the media for Gardasil, that most participants already had high levels of knowledge about HPV and the vaccine. The majority of unvaccinated women had heard of HPV (96.6\%) and Gardasil (91.4\%) and the average knowledge score for unvaccinated women was $6.96(\mathrm{SD}=1.26)$ out of a sample range of 4 to 9 . However, this is still lower than the average knowledge score of $7.56(\mathrm{SD}=1.15)$ for vaccinated women. The literature suggests that awareness of HPV has increased over the past decade (Gerend \& Magaloire, 2008; Baer et al., 2000; Yacobi et al., 1999). Moreover, HPV knowledge has not been a reliable predictor of HPV vaccine acceptance in previous studies (Boehner et al., 2003; Gerend \& Magaloire, 2008; Jones \& Cook, 2008).

\section{Relationship between HPV risk factors and vaccination intentions in} unvaccinated women. Contrary to the literature, none of the HPV risk factors which included sexual activity, occurrence of an abnormal pap smear, number of sexual partners, age of first intercourse and frequency of condom use with a new partner as well as in a committed relationship, predicted HPV vaccine intentions. Since the vaccine has been available for several years, the women with the most HPV risk factors may have already been vaccinated. Therefore, women who have not yet been vaccinated may not be persuaded to get the vaccine due to HPV risk factors if this is not a salient concern for them. 
In summary, perceived benefits appears to be an important factor that influences unvaccinated women to get the vaccine. However, HPV knowledge and HPV risk factors were not salient in predicting vaccination intentions. In order to further understand why some women get the vaccine and others do not, the next section explores which factors differentiate vaccinated from unvaccinated women.

\section{Findings Comparing Vaccinated and Unvaccinated Women}

HBM for vaccinated versus unvaccinated women. Another purpose of this study was to explore which HBM variables differentiated vaccinated from unvaccinated women. The majority of studies about the HPV vaccine have not included women who have been vaccinated (Gerend \& Magloire, 2008; Jones \& Cook, 2008; Kahn et al., 2003). Including vaccinated women is important since it increases the understanding of which factors positively influence vaccination and which factors serve as barriers. Since this study aimed to explore vaccination behavior, women who had already received the vaccine were asked to think back to when they were contemplating getting the vaccine and answer the questions according to their thoughts and perceptions at that time.

Unvaccinated women were asked about their current thoughts and perceptions about the vaccine.

The variables of HPV knowledge, cues to action and subjective norms were controlled for before examining the HBM variables. The findings from these variables are discussed in the next section. It was hypothesized that all of the HBM model variables would differentiate vaccinated and unvaccinated women but only self-efficacy was found to be a significant predictor. This finding partially supports the stated hypothesis and previous literature (Buchanan, 2008; Gerend \& Barley, 2008; Gerend et al., 2008). Self- 
efficacy remained significant (Wald $=9.05, p<.01$ ) and accounted for unique variance even after controlling for the other HBM variables (benefits, barriers, susceptibility and severity). Therefore, women who received the HPV vaccine reported higher self-efficacy about overcoming the barriers for vaccination as compared to unvaccinated women.

However, in contrast to what was hypothesized based on the previous literature, the HBM variables of perceived susceptibility, severity, barriers and benefits did not significantly predict HPV vaccination status. Only one other known study has used the HBM variables to explore vaccinated versus unvaccinated women and their results partially support the present findings (Allen et al., 2009). Allen et al. (2009) found that perceived benefits and barriers significantly differentiated these two groups of women, but perceived susceptibility and severity did not. Perceived susceptibility may not be a salient variable if the women who received the HPV vaccine did so as a preventative measure before they felt susceptible to HPV. Moreover, if women do not feel susceptible to HPV, then even if the perceived severity of the HPV is high, this factor may not influence their decision to get the vaccine. In the present study, vaccinated and unvaccinated women had very similar levels of perceived severity with mean scores of 33.57 and 32.42 respectively in a possible range from 6 to 42 . Both groups had relatively high levels of perceived severity which suggests that there were other factors besides perceived severity that caused some women to get the HPV vaccine and others to refrain.

The finding that perceived barriers was not a significant predictor was surprising given that fewer unvaccinated women reported having health insurance (89.7\%) and having health insurance that covered the HPV vaccine (60.3\%) as compared to vaccinated women (97.3\% and $83.3 \%$, respectively). Perceived benefits and barriers may 
not have been significant in the present study since other important variables were controlled for, like HPV knowledge, cues to action and subjective norms. Allen et al. (2009) included subjective norms in their analysis, but did not include cues to action or self-efficacy. Evidence of possible overlap between the HPM variables and other variables are reflected in low to medium significant correlations between all of the HBM variables and subjective norms. In addition, cues to action was significantly correlated with all of the HBM variables except for perceived severity. Thus, controlling for cues to action and subjective norms may have removed some of the predictive power of the HBM variables.

\section{Cues to action, subjective norms and HPV knowledge for vaccinated versus}

unvaccinated women. Cues to action, subjective norms and HPV knowledge were all found to significantly differentiate vaccinated from unvaccinated women. Cues to action (Wald $=7.98, p<.01$ ) and subjective norms (Wald=10.94, $p<.001)$ predicted unique variance even after controlling for the HBM variables. Therefore, women who received the HPV vaccine reported a greater number of cues from the environment about receiving the vaccine (e.g. Have you ever seen an ad on T.V. about the HPV vaccine (Gardasil)?) and reported that more people (e.g. friends, parents, partners) in their lives would support their decision to become vaccinated as compared to unvaccinated women. These findings are also supported by the literature. Previous research has explored cues to action in relation to HPV vaccination intentions, but not in terms of differentiating vaccination status. One previous study found that higher cues to action, such as knowing a close friend or relative with HPV, predicted greater HPV vaccination intentions (Jones \& Cook, 2008). Prior research on subjective norms and HPV vaccination status found that 
college women who planned to receive the vaccine sometime in the future and those who had already received the vaccine reported significantly higher subjective norms than women who had decided against the vaccine (Allen et al., 2009).

In the present study, vaccinated women also had significantly higher levels of HPV knowledge than unvaccinated women. This supports similar findings by Allen et al. (2009) and suggests that HPV is more salient for vaccinated women or that vaccinated women tend to be more informed than unvaccinated women. However, HPV knowledge did not significantly predict vaccination status when controlling for subjective norms and cues to action. Thus, although the two groups of women had different levels of HPV knowledge, this may not have been the most salient factor in influencing vaccinated women to receive the vaccine. Moreover, HPV knowledge and cues to action were moderately significantly correlated which may have reduced the predictive power of HPV knowledge.

HPV risk factors for vaccinated versus unvaccinated women. Certain sexual factors differentiated the vaccinated from unvaccinated women. For instance, significantly more vaccinated women reported being sexually active and having more sexual partners than unvaccinated women. These findings are consistent with the sexual risk factors that predicted vaccine intentions in previous studies (Gerend \& Magloire, 2008; Jones \& Cook, 2008; Boehner et al., 2003; Costar, 2008; Buchanan, 2008). The frequency of condom use with a new partner or in a committed relationship did not significantly differ between the two groups. However when looking only at the proportion of women who always use a condom, significantly more unvaccinated women reported always using a condom with a new partner as compared to vaccinated women. 
In fact, the number of vaccinated women who always use condoms in a new relationship (54.3\%) was surprisingly low. Other sexual risk factors did not differentiate the two groups, such as the occurrence of an abnormal pap smear and the mean age of first intercourse. Interestingly more vaccinated participants (10.7\%) reported being diagnosed with HPV as compared to unvaccinated women (5.2\%), even though the occurrence of an abnormal pap smear was the same for both groups (vaccinated $=20.7 \%$, unvaccinated $=$ 19.0\%). Since an abnormal pap smear generally implies the presence of HPV, this finding suggests that more vaccinated women knew or were told they had HPV as compared to unvaccinated women. In conclusion, vaccinated women appear to have more risky sexual behaviors in some areas, but not in others.

It is difficult to determine the implications that these findings may hold for receiving the HPV vaccine. On one hand, the decision to get the HPV vaccine could be seen as a positive health behavior that protects women from a harmful STD. Women who were aware that they were more at risk for contracting HPV due to their sexual behaviors may have been more engaged in health protective behavior by accurately assessing their risk and taking action to minimize that risk. Evidence from this study suggests that women with greater sexual risk behaviors reported higher perceived susceptibility for HPV. For instance, participants with an abnormal pap smear and more sexual partners reported higher perceived susceptibility. It is possible that getting the vaccine was a health-protective action based on their assessment of their risk or it may have been an action that gave them a false sense of protection. Vaccinated and unvaccinated women differed on sexual risk factors in some important ways, however, since the current study was not longitudinal in design, it is unclear how the vaccine plays a role in the sexual risk 
behaviors of participants. It seems that the number of sexual partners, current sexual activity and condom use will be important factors to explore in future studies on HPV vaccination.

In summary, self-efficacy, subjective norms, cues to actions, HPV knowledge and certain HPV risk factors differentiated vaccinated from unvaccinated women. The next section further describes the vaccinated sample and explores how satisfaction with receiving the vaccine and the length of time since vaccination may be related to the HBM variables.

\section{Findings about Vaccinated Women}

\section{Retrospective recall and time since vaccination for women who received the}

vaccine. The third purpose of the study was to examine vaccinated women since most research has only looked at unvaccinated women. Including women who have received the vaccine remains a relatively unexplored area and can help illuminate the reasons behind getting vaccinated. Vaccinated women were asked to think back to when they were contemplating getting the vaccine and answer the questions according to their thoughts and perceptions at that time. Since this involved retrospective recall, the amount of time that had passed since receiving the vaccine may have influenced their responses. Previous studies have found that retrospective reporting influences the perceived importance of HBM variables, where benefits and barriers are viewed as more salient and severity as less salient, as compared to prospective reporting (Harrison et al., 1992;

Brewer et al., 2007). Therefore, the amount of time since getting the vaccine was explored to see if it correlated with women's responses on the HBM variables (benefits, barriers, susceptibility and severity). It was found that the amount of time since 
vaccination did not correlate with benefits, barriers, susceptibility or severity. This suggests that women who had received the vaccine three years ago did not respond in a systematically different way than women who had received the vaccine several months ago.

\section{Relevance of the HBM variables for satisfaction with receiving the HPV}

vaccine among vaccinated women. Participants were generally satisfied with their decision to receive the vaccine, as indicated by the mean satisfaction level of 6.18 $(\mathrm{SD}=1.44)$ where scores ranged from 1 (low satisfaction) to 7 (high satisfaction). The relationship between satisfaction with receiving the HPV vaccine and the HBM variables was explored. Results indicate that there was a significant positive correlation between satisfaction with receiving the HPV vaccine and the HBM variables of severity and benefits. Participants who perceived more benefits from getting the vaccine and more severe consequences from HPV were more satisfied with their decision. Therefore, to a certain extent, participants' expectations about the vaccine were fulfilled. However, perceived barriers and susceptibility were not significantly correlated with satisfaction. This indicates that women's perception of the barriers to getting vaccinated and their susceptibility to HPV did not influence their satisfaction with their decision. Since vaccinated women were able to get the vaccine, perhaps they did not view the barriers as major obstacles or perhaps they viewed the vaccine as worth the sacrifice. This may explain why barriers did not influence their level of satisfaction. In addition, susceptibility may not have influenced women's satisfaction with getting the vaccine since vaccination would protect them from future susceptibility even if they did not perceive themselves as being currently susceptible to HPV. 
In summary, vaccinated women who reported higher levels of satisfaction with their decision to receive the HPV vaccine also reported higher perceived benefits of the vaccine and higher perceived severity of HPV. There was no relationship between the HBM variables and length of time since vaccination. The next section describes vaccinated and unvaccinated participants responses to open-ended questions about their decision to receive or not receive the vaccine.

\section{Open-Ended Questions}

Benefits and drawbacks of HPV vaccination. Another purpose of this study was to explore the factors that negatively and positively influence the decision to receive or not receive the HPV vaccine. Open-ended questions provided an opportunity to identify influential factors that might not be addressed by the Health Belief Model. Participants were asked, "What do you view as the greatest benefits of HPV vaccination?" Women described decreased anxiety and worry as well as prevention of HPV, genital warts and cervical cancer as the benefits of the vaccine. Prevention of HPV and its clinical sequelae was cited in the majority of responses for both vaccinated (76.6\%) and unvaccinated (73.1\%) participants. Women who gave this response thought that the greatest benefit of the vaccine was its role keeping them "safe from getting cervical cancer," giving them “protection against HPV," and "not getting genital warts." Women also described how the vaccine improves sexual health and prevents the spread of HPV to partners. Responses included "improvement of health" and "protecting myself from the possibility of contracting and then spreading it."

Decreased anxiety and worry was the next most common response for both vaccinated and unvaccinated women, although there were significantly more vaccinated 
participants (42.6\%) who cited this response as compared to unvaccinated $(38.3 \%)$. The vaccine provided women with peace of mind and a sense of comfort about their health. As one participant stated, the biggest benefit of the vaccine was "peace of mind. Knowing that I've done something else (besides abstinence) to help prevent HPV and cervical cancer. So if I do get it I won't have to feel like I didn't do everything I could to prevent it." In addition, women described feeling more in control of their health and not having to worry about contracting HPV as a result of the vaccine. One response included, "Not having to think about getting checked so often for cervical cancer."

Finally a small number of vaccinated women (2.1\%) and significantly more unvaccinated participants (11.5\%) stated no need or interest in the vaccine. One participant wrote:

I do not view any health benefits. I feel that people should practice safe sex and not be coerced into getting the Gardisil vaccine by their GYN. The vaccine has not been around long enough to see the long term health side effects.

Another participant described, "I do not see any benefits unless I decide to completely change my lifestyle and become sexually active with people whose health I don't know about." One of the women who had received the vaccine responded, "I don't really think there were any benefits for me because even after I had all the vaccines I still had an abnormal pap smear result whereas before getting the vaccines I had never had an abnormal result before."

Responses to these open-ended questions are similar to those found in the qualitative portion of Costar's (2008) study about primary reasons women would get the HPV vaccine. As with this study, the majority (68.5\%) of participants in Costar's (2008) 
study cited the benefits of prevention as the main reason to get vaccinated. The benefits of reducing worry were also evident in Costar's (2008) study, as well as a small number of participants $(5.2 \%)$ who had no wish to be vaccinated. In short, receiving the HPV vaccine has two main benefits - protection from HPV and reducing worry - that women should consider.

In order to determine drawbacks to vaccination, participants were asked, "What do you view as the greatest drawbacks of HPV vaccination?” In response participants described logistical concerns, stigma and embarrassment, not perceiving themselves at risk, perceiving no need, lack of interest or lack of knowledge about the vaccine, concerns about the efficacy and safety of the vaccine, and concerns about having to get a shot. Finally, some of the participants did not see any drawbacks to getting the vaccine.

The most common drawback for unvaccinated women was their concern about the efficacy and safety of the vaccine. Significantly more unvaccinated (53.8\%) cited this concern as compared to vaccinated women (25.4\%). Responses described worries that the vaccine had unknown side effects and had not been thoroughly tested or around long enough to be safe. As one participant responded the biggest drawback about the vaccine was, "the uncertainty about long term symptoms/consequences. The vaccination is relatively young. I don't want to sign up for something that may turn out to be a bad thing in a few years." Some thought that the vaccine "may not be completely effective" or would not be effective for non-virgins. As one participant wrote, "I heard it doesn't work once you've started having sex already." Several participants stated that receiving vaccinations went against their beliefs. One participant responded, "It's against my 
beliefs. I also believe that since it's still a fairly new vaccine, it could cause serious damage/side effects down the line that we can't yet predict."

A similar percentage of vaccinated $(29.7 \%)$ and unvaccinated $(26.9 \%)$

participants reported logistical concerns as a drawback to getting the vaccine. Responses indicated that they felt the vaccine was "expensive" and that receiving three shots was "time consuming." One participant wrote, "It is difficult to plan out the three shots in the appropriate time span." Out of the total sample, $10.6 \%$ of the participants had not received all three vaccines even though they were motivated to begin the vaccination. In addition, other women stated that they would not have been able to afford the vaccine without insurance. As one participant responded, "If my insurance hadn't approved it, I wouldn't have gotten it because I know it's pretty expensive."

Participants also described concerns about having to get a shot as a drawback to getting vaccinated. This seemed to be more of a concern for vaccinated participants since significantly more of them $(29.7 \%)$ cited this response than unvaccinated participants (15.4\%). Vaccinated participants described how receiving the shot was "painful" and complained of minor side effects from the vaccine, such as "soreness for a few days after receiving the shot" and vomiting after vaccination. Unvaccinated participants responded that receiving a shot would be painful and talked about having a fear of needles ("I hate needles").

Only unvaccinated participants described not perceiving themselves at risk for HPV $(13.5 \%)$ or having no need or interest in getting the vaccine $(9.6 \%)$.One reason seemed to pertain to women being in a monogamous relationship. Responses included, "no need with monogamous partner" and "waste of time and money, only important if 
my husband has HPV since he is my only partner." Other reasons for having no need for the vaccine involved not being sexually active. One participant stated, "Why do it now when I don't need it since I'm not having sex, until I am going to have sex it would just be a waste of time, especially seeing as how I'm nowhere near close to having sex."

Although very few vaccinated and unvaccinated participants described stigma and embarrassment as a drawback to getting the vaccine, significantly more unvaccinated participants $(7.7 \%)$ cited this concern as compared to vaccinated women (1.4\%). Unvaccinated women who endorsed this response wrote, "I'd have to tell my parents" and described the drawback of "having everyone around me thinking I'm at risk because I'm either sexually active or plan to be, when I'm not." Vaccinated participants reported "possible social stigma" around getting the vaccine.

The most common response from vaccinated participants was that there were no drawbacks to getting the vaccine. Significantly more vaccinated women (39.9\%) endorsed this response as compared to unvaccinated women (7.7\%). One participant wrote, "No drawbacks. It's worth it." Ten percent of women at first stated that there were no drawbacks, but then went on to describe a downside of the vaccine. The most common downside in this case was the pain associated with receiving a shot.

Responses to these open-ended questions are also similar to those found in the qualitative portion of Costar's (2008) study about primary reasons women would not get the HPV vaccine. Costar (2008) found similar themes of participants not perceiving themselves at risk and having no desire to get the vaccine. Moreover, some participants in Costar's (2008) study indicated that there was no reason not to get vaccinated, much like the women in this study who saw no drawbacks to vaccination. Some of Costar's (2008) 
categories were slightly different, but may still relate to the categories in this study. For instance, Costar (2008) found responses that described increased anxiety which may relate to concerns about the efficacy and safety of the vaccine and concerns about getting a shot that were described in this study. Participants in Costar's (2008) study also described how the benefits of vaccination do not outweigh the costs which may relate to the participants who reported logistical concerns in this study.

What factors led you to get vaccinated? Only vaccinated women answered this open-ended question with the aim of discovering which factors most influenced their decision to get the vaccine. There has been speculation about how to market the vaccine to women and the responses to this question could help clarify which areas to target. Findings indicate that the majority of participants $(79.3 \%)$ were influenced to get the vaccine by other people. Specifically, doctors or medical professionals (48.6\%) and mothers $(33.6 \%)$ were the people most commonly cited who influenced participants to get vaccinated. Participants also described how siblings or friends (15.0\%) and fathers $(9.3 \%)$ influenced them. This supports the finding that subjective norms, plays an important role in differentiating vaccinated from unvaccinated women. Subjective norms refer to how important people, such as parents, friends, doctors, siblings and significant others, view a certain health behavior. Therefore, having people accept and encourage health behaviors, such as getting the HPV vaccine, is perhaps the biggest factor that influences women to get vaccinated. Doctors and medical professionals appear to have a particularly strong influence $(48.6 \%)$ and a proactive approach by the medical community to encourage HPV vaccination could be paramount in the dissemination of the HPV vaccine. In addition, more vaccinated women had health insurance $(97.3 \%)$ and 
had insurance that covered the HPV vaccine $(83.3 \%)$ as compared to unvaccinated women ( $89.7 \%$ and $60.3 \%$, respectively). Therefore, doctors perhaps had more access to women with health insurance. Perhaps not surprisingly, mothers had more of an impact than fathers on participant's decision to get vaccinated. Women may be more comfortable talking to their mothers about HPV or their mothers may be in charge of the family's health more so than their fathers. In addition, mothers may themselves have worried about abnormal pap smears based on their past experience or knowing someone else who had one. Interestingly, friends and siblings (15.0\%) did not play as large a role as expected in influencing participants to get the vaccine. Participants may be hesitant to discuss the vaccine with their friends since HPV is associated with sexual behaviors or mothers and physicians may have had the first opportunity to influence women. In addition, many of the women may have been on their parents' health insurance and thus looked to their family rather than friends for support in getting the vaccine.

Vaccinated participants also cited other factors that led them to get vaccinated, including the desire to protect themselves from HPV, genital warts, or cervical cancer (35.7\%), perceiving themselves to be at risk for HPV (15.7\%), being influenced by advertisements or the media (11.4\%), and describing little or no downsides to receiving the vaccine (12.1\%). Some of the women who reported a desire to protect themselves stated wanting to "protect my sexual health in the long-run" and "prevent the spread of a common disease." Protection appeared to be a common factor that influences participants to get the vaccine and is theoretically related to the HBM variable of perceived benefits, which includes the vaccine's effectiveness in preventing HPV, genital warts and cervical cancer. Women who perceived themselves to be at risk for HPV cited not only sexual 
activity, but also having had an abnormal pap smear or having a family history of cancer. This category is related to the HBM variable of perceived susceptibility. The relatively low number of women who reported perceived risk as a reason for getting the vaccine may explain why perceived susceptibility did not differentiate vaccinated from unvaccinated women. Given the large amounts of Gardasil advertisements, it is surprising that so few women cited the media as a factor that led them to get the vaccine. Of the women who wrote about the media, commercials were the most common type of advertisements cited in participants' responses and these commercials may have reinforced the importance of the vaccine as well as normalized the need to get vaccinated. In summary, support and encouragement by other people, especially doctors and mothers, was the biggest factor that influenced participants to get the HPV vaccine. The desire for protection against HPV and its clinical sequelae also seemed to motivate women to get vaccinated. This information can be utilized to promote the vaccine to women and reach those in their late teens and early twenties before they are no longer eligible for HPV vaccination.

What factors keep you from getting vaccinated? Only unvaccinated women answered this open-ended question with the aim of discovering which factors were hindering them from seeking the vaccine. Most of the reasons why unvaccinated women had not yet received the vaccine were the same as the drawbacks described by the whole sample. Almost half of the responses indicated that participants did not perceive themselves at risk for HPV (41.5\%) and/or were concerned about the efficacy and safety of the vaccine $(41.5 \%)$. All of the women who did not perceive themselves at risk reported that they were either not sexually active or were in a monogamous relationship. 
The perception that women should not get the HPV vaccine until they are sexually active is problematic since the purpose of the vaccine is to prevent HPV before individuals are exposed. In fact, the vaccine has been recommended for 11 to 12 year old girls in order to protect them before they become sexually active. The participants who endorsed this response seemed to be making a decision about their current level of risk rather than considering how their circumstances might change, such as becoming sexually active or having a new sexual partner in the future. The participants who reported concerns about the efficacy and safety of the vaccine wrote that it was "too young to be entirely trusted" and worried about "unknown, long-term consequences." These participants seemed to think that the vaccine was potentially dangerous and not worth the risk. Therefore, women's perceptions of their current risk and of the vaccine appeared to be the biggest factors keeping them from getting vaccinated.

Almost a quarter $(24.5 \%)$ of the responses also described logistical concerns, most $(84.6 \%)$ of which mentioned the cost of the vaccine and/or that their insurance did not cover it. This suggests that more women would become vaccinated if the shots were more affordable, either through better insurance coverage or through a lower overall cost for the vaccine.

Equal numbers of participants cited no need or interest in the vaccine (11.3\%) and thinking that the vaccine won't help non-virgins (11.3\%). Some women who felt no need to get the vaccine reported "inadequate knowledge of what HPV is" or stated, "I do not get vaccinations." Others stated that the vaccine was no longer effective since they were already sexually active or that getting the vaccine was pointless since they had already contracted HPV. Additional factors that kept participants from getting vaccinated 
included concerns about getting a shot (9.4\%) and worries about stigma and embarrassment (1.9\%). Some participants stated that they had a fear of needles or a phobia to receiving vaccines. Only one participant cited stigma and embarrassment by stating that she was worried about her "parents finding out."

In conclusion, the most common factors that kept participants from getting vaccinated were their concerns about the vaccine's efficacy and safety, and their perception of not being at risk for HPV. However, logistical concerns, such as the cost of the vaccine, were also indicated as hindering women from receiving the vaccine. Although the large majority of unvaccinated participants had heard of HPV (96.6\%) and the HPV vaccine $(91.4 \%)$ before the study, it is clear that myths about both still abound, including the idea that the vaccine is no longer effective for non-virgins. Efforts to dispel misinformation about the vaccine and educating women about their susceptibility to HPV represent important steps in promoting the HPV vaccine.

\section{Overall Summary of Findings}

The Health Belief Model (benefits, barriers, susceptibility and severity) and other social cognitive variables (subjective norms, cues to action, self-efficacy, HPV knowledge and sexual risk factors) were originally presented in this study to help predict which factors would influence women to receive the HPV vaccine. Two groups of women, HPV vaccinated and unvaccinated, were examined both separately and then in comparison to each other. For unvaccinated women, only perceived benefits accounted for unique variance in predicting their intentions to get the HPV vaccine. This suggests that women who view the HPV vaccine as more effective and beneficial in protecting them against HPV and its consequences have higher intentions of getting vaccinated. 
However, unvaccinated women qualitatively reported concerns about the vaccine's efficacy and safety as factors that keep them from getting the vaccine. In addition, many of the women qualitatively reported that they did not perceive themselves to be at risk for HPV because they were either in a monogamous relationship or were not sexually active. The results indicate that more unvaccinated women $(53.4 \%)$ reported being married or in a monogamous relationship than vaccinated women (44.0\%). In addition, more unvaccinated women $(39.7 \%)$ reported NOT being currently sexually active as compared to vaccinated women $(17.3 \%)$. Therefore unvaccinated women seemed to consider their sexual situation with an apparent emphasis on the here and now or near future rather than in the long-term. These factors may account for why these participants had not yet received the HPV vaccine. However certain aspects may eventually persuade unvaccinated women to get the vaccine, such as additional evidence regarding the safety and efficacy of the vaccine, changes in women's sexual status (i.e. becoming sexually active or ending a current relationship), or consideration of the beneficial aspects of the vaccine.

For vaccinated women, the majority were generally satisfied with their decision to receive the vaccine. Interestingly, participants qualitatively reported that the biggest factor which led them to get the vaccine was support and encouragement by other people, especially their doctors and mothers. This suggests that social support may be an important factor in helping unvaccinated women decide to receive the vaccine. In addition, participants qualitatively reported that the desire for protection against HPV, genital warts and cervical cancer also strongly motivated them to get the vaccine. 
Finally, vaccinated women were compared to unvaccinated women on a number of factors, including the Health Belief Model variables. Surprisingly, none of the four main components of the HBM (perceived benefits, barriers, susceptibility and severity) differentiated vaccinated from unvaccinated women. Instead, other variables such as selfefficacy for receiving the vaccine, cues to action and subjective norms all accounted for unique variance in predicting vaccination status. Vaccinated women also had significantly higher knowledge about HPV and the vaccine as compared to unvaccinated women, but this finding disappeared when cues to action and subjective norms were taken into consideration. This suggests that women's decision to get the vaccine involves a complex interplay of factors that are not necessarily captured by a rational, cost-benefit model like the HBM. Vaccinated women reported higher self-efficacy for receiving the vaccine (although this is based on retrospective recall), more positive cues for receiving the vaccine and more approval from their social network for receiving the vaccine as compared to unvaccinated women. Thus external factors seem to play a key role in influencing HPV vaccination behavior. When a woman's environment supports HPV vaccination, through encouragement by others and reminders of the vaccine in daily life, this may lead to increased knowledge about HPV as well as increased confidence in receiving the vaccine. Moreover, women who have support from their environment may find it easier to overcome any barriers associated with vaccination. External factors may be particularly salient with this health behavior since HPV vaccination has had a large amount of media coverage and controversy associated with it. In addition, since the vaccine is associated with sexual behaviors and has been marketed to young girls, parents have been especially involved in this health behavior. Although the sample in this study 
was older than the target population for the vaccine, parents of these participants may still have been more aware and instrumental in their decision due to the media coverage. Finally, doctors may have been instrumental in promoting the vaccine since they may benefit financially from giving vaccinations and they may also realize that the HPV vaccine has the potential to completely eliminate cervical cancer. The HBM variables (benefits, barriers, susceptibility and severity) did account for significant variance, but not for unique variance when considering them along with the other variables (selfefficacy, cues to action and subjective norms). Therefore, women may still consider the benefits and barriers of HPV vaccination as well as the severity of HPV and their susceptibility to it. However, the more external variables of subjective norms, selfefficacy and cues to actions appear to be more important in influencing women's decision to receive or not receive the vaccine.

Vaccinated women also reported more HPV risk factors such as being sexually active and having more sexual partners than unvaccinated women. In addition, unvaccinated women reported always using condoms with new partners as compared to vaccinated women. It is possible that women were aware of their risk for contracting HPV or made an accurate risk assessment based on their sexual behaviors which prompted them to get the vaccine. Women who perceived themselves to be at risk for HPV may have been more influenced by cues to action, such as advertisements, and more persuaded by their doctor. On the other hand, receiving the vaccine may have been an action that gave women a false sense of protection. In general, parents have cited concerns that the vaccine will promote risky sexual behavior. However, these conclusions 
cannot be drawn from this study since it is not longitudinal and the role that the vaccine plays in sexual behaviors is still unknown.

In summary, the Health Belief Model can be useful for determining women's intentions to get the HPV vaccine. In particular, the perceived benefits of receiving the HPV vaccine seems to play an important role. Yet many unvaccinated women viewed the vaccine as having benefits but not for them at the moment. When considering actual vaccination behavior, such as comparing vaccinated versus unvaccinated women, factors that go above and beyond the four main components of the Health Belief Model, such as self-efficacy, subjective norms, cues to action and HPV knowledge become salient. Additionally, this study contributes to HPV vaccination research by qualitatively exploring factors that keep women from and lead women to getting the vaccine.

\section{Limitations}

This study has several limitations. The primary limitation includes the crosssectional design of the study. In order to fully test the health belief model, a longitudinal design is required since the goal is to predict future behavior. While longitudinal research is important and necessary, it was beyond the scope of this study. Instead, unvaccinated reported on their current perceptions of the vaccine, whereas vaccinated women were asked to retrospectively report on their thoughts and perceptions when they were contemplating getting the vaccine. Since retrospective recall was involved, the amount of time that had passed since vaccination may have influenced their responses. Participants reported an average of 16.9 months $(\mathrm{SD}=11.0)$ since vaccination with a range of 1 to 44 months. Therefore, participants had to remember what their perceptions of the vaccine were on average one and a half years ago. This may have biased the results in that they 
reported more benefits and fewer barriers than they actually perceived at the time. In order to test for this, the amount of time since vaccination was compared to the HBM variables and no significant correlations were found. However, participants may still be more biased in their accounts of their perceptions than unvaccinated women.

Another limitation of the study includes the lack of standardization for the health belief model measures. These measures aren't standardized since the scale items depend on the behavior and the population of interest. Although some valid and reliable HBM measures exist, they do not relate specifically to the HPV vaccine. In order to resolve this limitation, several steps were taken. First, experts were consulted to ensure the construction of conceptually and methodologically sound measures. Second, an exploratory factor analysis with oblimin rotation was conducted to see if items loaded onto the hypothesized HBM variable. Each item was examined for sufficient loading (.30 or higher) on the hypothesized factor and for cross-loadings on more than one factor. The number of factors to retain for each HBM variable was also explored. However, there were not enough items in the barriers scale to thoroughly explore the possibility of multiple factors. Third, Cronbach alphas were calculated and found to be above 0.72 for all scales except for perceived barriers which was 0.54 for vaccinated women. Although data from both vaccinated and unvaccinated participants loaded in a similar manner onto the factors, there were some minor differences between the two groups which made it difficult to decide which items to eliminate in designing the final measure. In addition, unvaccinated women were answering items based on how they currently felt, whereas vaccinated women were retrospectively reporting about how they felt at the time of making the decision to get the vaccine. 
Finally, this study is limited by the fact that the participants are enrolled in higher education settings and therefore may not be representative of all women in the 18-26 age group. For instance, college women are likely to have greater access to health information and services compared with those who are not in school (Allen et al., 2009). This has implications for external validity since it restricts the generalizability of the results. In addition, there may be a sampling bias since participants were primarily recruited from sororities and psychology or education classes. Therefore, the generalizability of this study to college-age women in general is restricted. In order to increase the heterogeneity of the sample, participants were recruited as much as possible from universities in different regions of the country.

\section{Implications for Research and Practice}

This study was conducted with the aim of understanding the role of the Health Belief Model in college-age women's vaccination behavior for the human papillomavirus vaccine. The current study was one of only a few studies to include all of the components of the HBM such as perceived benefits, barriers, susceptibility and severity as well as self-efficacy and cues to action. Other social cognitive variables were of interest in predicting vaccination behavior, including subjective norms, HPV knowledge, and HPV sexual risk factors. This was also one of the few studies to look at both vaccinated and unvaccinated women and based on this, future studies might consider conducting longitudinal studies that explore which factors predict vaccination behavior rather than just vaccination intentions.

Getting the HPV vaccine is a positive health-protective behavior for most young women today. Recently, all of the strains of HPV that account for nearly all cervical 
cancers have been identified in order of importance, so the HPV vaccine will soon protect against all of these strains. Given the importance of HPV vaccination, future studies should employ a longitudinal design to study which variables predict vaccination uptake. The present study explored vaccination behavior retrospectively by having vaccinated women think back to when they were contemplating getting the vaccine, whereas unvaccinated women answered questions based on their current thoughts and perceptions around the vaccine. This retrospective reporting may have introduced some bias, whereas a longitudinal study would measure current perceptions for all participants. In addition, the current study explored unvaccinated participants intentions to receive the HPV vaccine in the future. Although intentions may be correlated with behavior, it is still unknown what would actually lead unvaccinated women to participate in vaccination uptake. A longitudinal study would allow for full testing of the factors that predict HPV vaccination behavior. It would be important to study which variables predict vaccination uptake in order to determine the factors that should be emphasized in promotion of the vaccine.

Future research should explore how to increase both social and informational environmental support for receiving the vaccine as well as increase the self-efficacy of unvaccinated women to overcome barriers for vaccination. Although each individual woman should assess her situation and decide for herself whether or not to get the HPV vaccine, environmental support can make it easier for women to become vaccinated. Our results indicate that self-efficacy represents an important variable in differentiating vaccinated from unvaccinated women. Specifically, self-efficacy in this study referred to confidence in one's ability to overcome barriers for HPV vaccination. Therefore, 
vaccinated women had higher self-efficacy regarding pushing past perceived barriers as compared to unvaccinated women. Vaccinated women also reported higher subjective norms (e.g. parents, friends, doctor approved of the vaccine.) and cues to action (e.g. knowing someone who received the vaccine, seeing an ad on TV about the vaccine) as compared to unvaccinated women, suggesting that their environment was more instrumental to receiving the vaccine. In addition, a link may exist between self-efficacy and environmental support and future research should explore this connection in order to determine how these factors interact to promote vaccination.

The scales used to measure the HBM variables in this study were created by the researcher and require further validation. While the measures were used in both the vaccinated and unvaccinated samples, further testing would provide additional validation that these measures are appropriate for both samples. Future research should also test these measures in populations other than college-age women. In the current study, there were not enough items in the barriers scale to thoroughly explore the possibility of multiple factors. Therefore, additional items should be added to the scales in order to determine whether subscales exist within the HBM variables.

In future studies, HPV sexual risk factors, such as number of sexual partners, current sexual activity and condom use, will be important factors to explore in relation to HPV vaccination. The current study found that more vaccinated women reported being sexually active, having more sexual partners and using condoms less frequently than unvaccinated women. Future research should attempt to determine the implications that these findings may hold for receiving the HPV vaccine. For instance, did vaccinated women's risky sexual behaviors increase their desire to protect themselves through HPV 
vaccination or did the vaccine give these women a false sense of security and lead to increased sexual behaviors? These would be important questions to answer, particularly in light of parents' concerns that the vaccine promotes promiscuity. The vaccine is targeted for young girls (age 11-12) in order to protect them from HPV before they become sexually active since they may not know whether their future partners have HPV. Many girls and women never really know their lifetime potential risk of exposure since their partners may not even be aware if they have HPV or not. Unvaccinated women reported that they did not perceive themselves to be at risk for HPV due to not being currently sexually active or being in a monogamous relationship. Therefore, women seem to base their vaccination decisions on their current level or risk rather than on their potential risk level in the future (if they become sexually active or if they have new sexual partners). Further research should explore women's perception of risk for HPV and how this influences their decision to become vaccinated.

This study was conducted at a time where college-aged women could not have received the vaccine during the target age range of 11-12 years since it was not yet available. Therefore, these women made the decision whether or not to become vaccinated and were also influenced by significant others (parents, doctors and friends). In the future, females will likely get the vaccine as a routine childhood vaccination and will not need to make the decision about getting it in young adulthood. This study represents a point in time that will not really exist in the future. However, the results from this study can provide important information for studying health protective actions in general beyond the HPV vaccine. For instance, this study suggests that factors beyond the traditional HBM variables (benefits, barriers, susceptibility and severity), such as self- 
efficacy, cues to action and subjective norms play an important role in influencing vaccination behavior. Since HPV vaccination represents a behavior that protects against sexual risk, these findings may apply to other sexual protection behaviors, such as condom use. Future studies should incorporate variables beyond the traditional HBM factors in exploring protective sexual behaviors.

In terms of practice, health professionals appear to hold great influence over college-age women's decision to receive the vaccine. In this study, the majority of vaccinated women reported that doctors or medical professionals influenced them to get the vaccine. This suggests that health professionals can play a large role in increasing women's awareness of the vaccine and promoting its uptake. Many participants also indicated that their mothers influenced them to receive the vaccine. Thus, health professionals should not only educate college-age women about the vaccine, but also inform families and parents about the benefits of HPV vaccination. Education about HPV and the vaccine appears to be an important factor since vaccinated participants had higher HPV knowledge than unvaccinated participants. This suggests that being informed about HPV and the vaccine can help women realize the importance of getting vaccinated and may dispel misconceptions they have about the vaccine. The study's open-ended questions revealed that unvaccinated women still hold misconceptions about the vaccine, such as believing that the vaccine is ineffective for non-virgins. Moreover, unvaccinated participants cited these misconceptions as reasons not to get the vaccine. Therefore, health professionals are well positioned to educate women and their families about HPV and the vaccine and correct any misconceptions they might have. 
There has also been speculation about how to market the vaccine and this study helps clarify which areas to target for college-aged women. Specifically, unvaccinated participants had higher intentions to receive the vaccine when they perceived greater benefits to receiving the vaccine. Moreover, the open-ended questions revealed that the majority of participants thought the greatest benefit of vaccination was prevention of HPV and its clinical sequelae. Therefore, health messages should focus on the benefits of HPV vaccination and especially emphasize the efficacy of the vaccine in protecting against genital warts and cervical cancer. In addition, many unvaccinated participants held concerns about the safety of the vaccine or did not perceive themselves to be at risk for HPV. In order to address these concerns, health messages should include information about research on the vaccine and include the factors that place women at risk for contracting HPV, such as skin-to-skin contact. Moreover, the HPV vaccine has recently been approved for males and can potentially protect them against HPV-related cancers such as oral, penile and anal. This makes getting the vaccine something that not only protects women but protects their partners - male or female. Health messages should consider emphasizing how the vaccine can prevent the spread of HPV to partners since this might help them understand that the implications of vaccination goes beyond their own health.

Finally, given the negative psychological and physical effects of being diagnosed with HPV, counseling psychologists should advocate for making the HPV vaccine more available and affordable to college-aged women. Many unvaccinated participants expressed concerns about not being able to afford the vaccine since they did not have health insurance or their insurance did not cover it. In addition, some vaccinated women 
reported that they were only able to get the vaccine because their parents agreed to pay for it. Given the ability of this vaccine to prevent painful genital warts and cervical cancer, it is important to make it available to all women who would benefit from it. It seems that clinics on campuses and in communities could make the barriers of cost and inconvenience less problematic.

In summary, this study demonstrated a significant relationship between the HBM variable of perceived benefits and HPV vaccination intentions among women who had not yet received the vaccine. In addition, self-efficacy, subjective norms and cues to action were found to significantly differentiate vaccinated from unvaccinated women. Future research and a longitudinal study are needed to better identify the factors that lead to HPV vaccination behavior. This information can then be used to better inform interventions and health messages about the HPV vaccine which can lead to increased vaccination uptake. The current study highlighted the need to move beyond the traditional Health Belief Model when understanding the decision to receive the HPV vaccine. For instance, variables that were not a part of the traditional HBM, including cues to action, subjective norms and self-efficacy, were significant predictors of vaccination status. The use of open-ended questions allowed participants to describe positive and negative aspects of vaccination as well as the factors that led them to or kept them from getting the vaccine in their own words, such as by noting that:

Two of my close friends have the strands of HPV that cause cervical cancer and genital warts. I also contracted a strand of HPV right after the first shot that has no symptoms, and went through additional testing to verify that it was HPV. I 
wanted to make sure that I was protected against the most dangerous kinds, since the majority of HPV is symptomless and does not have long term effects.

In short, this study helped contribute to a greater awareness of the multifaceted dimensions of deciding whether or not to receive the human papillomavirus vaccine, including external variables such as subjective norms and cues to action that can help support women in overcoming any barriers they face in becoming vaccinated. 
Appendix A

$\underline{\text { Demographics }}$

1. Age:

2. Gender: female male

3. Ethnicity/Race:
African-American/Black
Latina/Hispanic
Middle-Eastern/Arab
Biracial/Multiracial
Other (please specify):

Asian-American/Pacific Islander White/European Descent

Asian Indian/ Pakistani

Native American/Native Alaskan

4. Current relationship status:

\section{Single Married Divorced Widowed \\ Committed Relationship (6 months or longer) Other}

5. Current year of undergraduate study:

6. Do you currently have health insurance, either on your own or under the insurance plan of another person?

YES NO

7. Does your health insurance cover the HPV vaccine? (if not applicable, skip this question)

YES NO

8. How many times over the past two years have you visited a gynecologist for a regular examination (including a Pap smear test)?

9. Before taking this survey, had you ever heard of HPV (human papillomavirus)? YES NO

10. If yes, how did you first hear about HPV?
a. Health care provider
b. A friend
c. A family member
d. Television or radio
e. Internet
f. Newspaper or magazine 
g. At school

h. Other (specify):

i. I don't remember

11. Before taking this survey, had you ever heard of the HPV vaccine called Gardasil?
a. Yes
b. No
c. I don't know

12. If yes, how did you first hear about the HPV vaccine?
a. Health care provider
b. A friend
c. A family member
d. Television or radio
e. Internet
f. Newspaper or magazine
g. At school
h. Other (specify):
i. I don't remember 


\section{Appendix B}

\section{Sexual Risk Factors}

In order to further our knowledge of reactions to a vaccine for a sexually transmitted disease, it is important that we ask questions pertaining to the health habits and sexual histories of the participants taking the questionnaire. Therefore, although some of the following information may be personal in nature, we ask that you answer as truthfully as possible.

1. Are you currently sexually active (defined, for the purpose of this study, as engaging in penis/vagina or penis/anus intercourse)?

YES

NO

2. Have you ever engaged in penis/vagina intercourse? YES

NO (If you have never been sexually active, please skip to question \#14)

3. Have you ever engaged in anal (penis/anus) intercourse?

4. At what age did you first engage in penis/vagina intercourse?

5. With how many partners have you engaged in penis/vagina intercourse?

6. At what age did you first engage in penis/anus intercourse? (if not applicable, skip this question)

7. With how many partners have you engaged in penis/anus intercourse? (if not applicable, skip this question)

8. How often do you use a condom when you have penis/vagina intercourse with a new partner?

\section{ALWAYS USUALLY SOMETIMES NEVER}

9. How often do you use a condom when you have penis/anal intercourse with a new partner? (if not applicable, skip this question)

\section{ALWAYS USUALLY SOMETIMES NEVER}

10. How often do you use a condom when you have penis/vagina intercourse with a partner with whom you are in a committed relationship (6 months or longer)?
ALWAYS
USUALLY
SOMETIMES
NEVER

11. How often do you use a condom when you have penis/anal intercourse with a partner with whom you are in a committed relationship (6 months or longer)? (if not applicable, skip this question)

\section{ALWAYS USUALLY SOMETIMES NEVER}

12. What is your usual method of birth control?

13. Have you ever had an unintended pregnancy? YES NO

14. Have you ever been diagnosed with the human papillomavirus (HPV)? YES NO 
15. Have you ever had an abnormal Pap smear test (e.g. atypical cervical cells)? YES NO

16. Have you ever been diagnosed with a sexually transmitted disease? YES NO If yes, please indicate which disease(s):

17. Have you ever been tested for a sexually transmitted disease? YES NO

18. Have you ever been diagnosed with atypical or precancerous cervical cells or cervical cancer? YES NO 
Appendix C

The human papillomavirus (HPV) is a sexually transmitted disease. A vaccine called Gardasil that protects against four types of human papillomavirus (HPV) was approved by the Food and Drug Administration (FDA) in June 2006 has since been available to the public. The HPV vaccine will be referred to as Gardasil in the following items.

Please answer the following questions regarding your thoughts about HPV and Gardasil. If you have already received Gardasil, please think back to when you were contemplating getting the vaccine and answer the questions according to your thoughts and perceptions at that time. If you have not received the Gardasil, please answer the questions according to your current thoughts and perceptions. Please answer these questions regardless of whether or not you are currently sexually active.

1. What are your chances of contracting HPV if you didn't get Gardasil? $\begin{array}{lllllllllll}0 \% & 10 \% & 20 \% & 30 \% & 40 \% & 50 \% & 60 \% & 70 \% & 80 \% & 90 \% & 100 \%\end{array}$

2. How likely is it that the benefits of Gardasil outweigh the potential side effects?

Unlikely

3. I would be embarrassed to let others know that I have HPV if I found out that I contracted the disease.

Strongly Disagree

1

4. Getting HPV could hinder my ability to become pregnant.

Strongly Disagree

1

3

4

5

Strongly Agree

$6 \quad 7$

5. Gardasil is effective in preventing an HPV infection.

Strongly Disagree

12

3

4

5

Strongly Agree

6. What are your chances of developing cervical cancer if you didn't get Gardasil? $\begin{array}{lllllllllll}0 \% & 10 \% & 20 \% & 30 \% & 40 \% & 50 \% & 60 \% & 70 \% & 80 \% & 90 \% & 100 \%\end{array}$ 
7. It would be difficult for me to ask for Gardasil because it's associated with an STD.

Strongly Disagree

12

3

4

Strongly Agree

5

7

8. Gardasil is effective in preventing genital warts.

Strongly Disagree

12

3

4

Strongly Agree

5

$6 \quad 7$

9. In your opinion, how expensive is Gardasil?

Very Inexpensive

12

3

4

Very Expensive

10. If I contracted HPV, it could hinder my present relationship.

Strongly Disagree

12

3

4

5

Strongly Agree

$6 \quad 7$

11. One of the benefits to getting Gardasil, is feeling that I've done everything I can do to protect myself against HPV.

Strongly Disagree

12

3

4

Strongly Agree

12. Having to get multiple doses of Gardasil is time consuming.

Strongly Disagree

12

34

5

Strongly Agree

13. Gardasil is effective in preventing cervical cancer.

Strongly Disagree

12

34

5

Strongly Agree

$\begin{array}{llll}5 & 6 & 7\end{array}$

14. What are your chances of contracting genital warts if you didn't get Gardasil? $\begin{array}{lllllllllll}0 \% & 10 \% & 20 \% & 30 \% & 40 \% & 50 \% & 60 \% & 70 \% & 80 \% & 90 \% & 100 \%\end{array}$

15. Getting Gardasil is painful.

Strongly Disagree

Strongly Agree 
16. Gardasil is effective in preventing some types of oral cancer.

Strongly Disagree
12
3
4

Strongly Agree

17. If I contracted HPV, it could hinder my future relationships.

Strongly Disagree

12

3

4

Strongly Agree

18. In your opinion, how likely is it that Gardasil causes significant side effects? Very Unlikely

12

3

4

5

$\begin{array}{cc}\text { Very } & \text { Likely } \\ 6 & 7\end{array}$

19. What are your chances of contracting genital warts if you didn't get Gardasil compared to other college-aged women?

$\begin{array}{lllllllllll}0 \% & 10 \% & 20 \% & 30 \% & 40 \% & 50 \% & 60 \% & 70 \% & 80 \% & 90 \% & 100 \%\end{array}$

20. Gardasil is effective in preventing the spread of HPV to partners.

Strongly Disagree

12
3

4

Strongly Agree

5

$6 \quad 7$

21. I would worry about what my partner or future partners would think if I got Gardasil.

Strongly Disagree

12

3

4

Strongly Agree

22. Getting Gardasil is important for my health.

Strongly Disagree

12
3

4

Strongly Agree

$\begin{array}{lll}5 & 6 & 7\end{array}$

23. What are your chances of contracting HPV if you didn't get Gardasil compared to other college-aged women?

$$
\begin{array}{lllllllllll}
0 \% & 10 \% & 20 \% & 30 \% & 40 \% & 50 \% & 60 \% & 70 \% & 80 \% & 90 \% & 100 \%
\end{array}
$$

24. If other people knew I received Gardasil, I would be embarrassed. 
Strongly Disagree

12

3

4

Strongly Agree

25. If I contracted HPV, it could harm my future health.

Strongly Disagree

12

3

4

$5 \quad$ Strongly Agree

26. One of the benefits to getting Gardasil is peace of mind about my health.

Strongly Disagree

12

3

4

Strongly Agree

5

$6 \quad 7$

27. What are your chances of developing cervical cancer if you didn't get Gardasil compared to other college-aged women?

$$
\begin{array}{lllllllllll}
0 \% & 10 \% & 20 \% & 30 \% & 40 \% & 50 \% & 60 \% & 70 \% & 80 \% & 90 \% & 100 \%
\end{array}
$$

28. Getting Gardasil would go against my beliefs.

Strongly Disagree

12
3

4

Strongly Agree

29. If I found out I had contracted HPV, I would be devastated.

Strongly Disagree

1
3

4
Strongly Agree

5

Perceived Severity Items: 3, 4, 10, 17, 25, 29

Perceived Susceptibility Items: 1, 6, 14, 19, 23, 27,

Perceived Benefits: 2, 5, 8, 11, 13, 16, 20, 22, 26

Perceived Barriers: 7, 9, 12, 15, 18, 21, 24, 28 


\section{Appendix D}

\section{Self-Efficacy}

Given your answer to the above question (\#__), how confident are you that you could get Gardasil?

Not Confident

12

3

4

5

Very Confident

6

7 


\section{Appendix E}

\section{Cues to Action}

1. Have you ever known anyone who has had HPV?

$$
\text { Never } \quad 3-2 \text { people } \quad 3-4 \text { people } \quad 5+\text { people }
$$

2. Have you ever known anyone who has had cervical cancer?

$$
\text { Never } \quad 1-2 \text { people } \quad 3-4 \text { people } \quad 5+\text { people }
$$

3. Has a health care provider ever recommended that you receive the HPV vaccine (Gardasil)?

$$
\text { Never } \quad 3-2 \text { times } \quad 3 \text { - } \quad 5+\text { times }
$$

4. Has a health care provider made you aware of the HPV vaccine's (Gardasil) availability?

$$
\text { Never } \quad 3-2 \text { times } \quad 3-4 \text { times } \quad 5+\text { times }
$$

5. Did you learn about HPV in any of your health education classes in either college or high school? If so, how many times did you learn about it?
Never
$1-2$ times
$3-4$ times
$5+$ times

6. Have you ever known anyone who has had negative side effects from a vaccine?

$$
\text { Never } \quad 3-2 \text { people } \quad 3-4 \text { people } \quad 5+\text { people }
$$

7. Have you ever known anyone who has received the HPV vaccine (Gardasil)?

$$
\text { Never } \quad 1-2 \text { people } \quad 3-4 \text { people } \quad 5+\text { people }
$$

8. Have family members or friends talked to you about receiving the HPV vaccine (Gardasil)?

$$
\text { Never } \quad 3-2 \text { times } \quad 3-4 \text { times } \quad 5+\text { times }
$$

9. Have you ever seen an ad on TV about the HPV vaccine (Gardasil)?

$$
\text { Never } \quad 3-2 \text { times } \quad 3-4 \text { times } \quad 5+\text { times }
$$

10. Have you ever seen an ad in a magazine about the HPV vaccine (Gardasil)?
Never
$1-2$ times
$3-4$ times
$5+$ times

11. Have you ever seen a brochure about the HPV vaccine (Gardasil)?
Never
$1-2$ times
$3-4$ times
$5+$ times 


\section{Appendix F}

\section{Subjective Norms}

1. If they knew about the HPV vaccine (Gardasil), most people whose opinions I value would approve/disapprove of me getting vaccinated against HPV.

Strongly Disapprove

1

2
3
4
Strongly Approve

5

2. If they knew about the HPV vaccine (Gardasil), my friends would approve/disapprove of me getting vaccinated against HPV.

Strongly Disapprove

1
Strongly Approve

$5 \quad 6 \quad 7$

3. If they knew about the HPV vaccine (Gardasil), my parents would approve/disapprove of me getting vaccinated against HPV.

Strongly Disapprove

12
3
4

5
Strongly Approve

$6 \quad 7$

4. If they knew about the HPV vaccine (Gardasil), my partner would approve/disapprove of me getting vaccinated against HPV.

Strongly Disapprove

1
3
4
Strongly Approve 5

5. If they knew about the HPV vaccine (Gardasil), my doctor would approve/disapprove of me getting vaccinated against HPV

Strongly Disapprove
3
4
Strongly Approve

5
$6 \quad 7$

6. If they knew about the HPV vaccine (Gardasil), most women who are important to me would get themselves vaccinated against HPV if they were at risk.

Strongly Disagree

12
3
4
Strongly Agree
6 
Appendix G

HPV Knowledge

Directions: Please answer the following questions about the human papillomavirus (HPV) and the HPV vaccine (Gardasil) to the best of your knowledge.

1. Which of the following health issues are related to HPV? (Please select all that apply.)
a. Cervical cancer
b. Heart disease
c. HIV
d. Genital warts
e. I don't know

Answer: a) cervical cancer, d) genital warts

2. How do you think HPV is transmitted or spread?
a. Coughing or sneezing
b. Genital skin-to-skin contact
c. Contact with bodily fluids (blood)
d. I don't know

Answer: b) genital skin-to-skin contact

3. How can HPV infection be prevented? (Please select all that apply.)
a. By practicing abstinence
b. By taking antibiotics
c. By using condoms
d. By being vaccinated
e. I don't know

Answer: a) by practicing abstinence, d) by being vaccinated

4. The HPV vaccine (Gardasil) may prevent which of the following. (Please select all that apply.)
a. Genital warts
b. Cervical cancer
c. AIDS
d. Herpes
e. I don't know

Answer: a) genital warts, b) cervical cancer

5. What is the main side effect associated with the HPV vaccine (Gardasil)?
a. Vomiting
b. Soreness at the site where the shot is given
c. Headache
d. Joint pain 
e. I don't know

Answer: b) soreness at the site where the shot is given
6. HPV affects only women.
TRUE
FALSE

Answer: FALSE

7. HPV can occur without symptoms. TRUE FALSE

\section{Answer: TRUE}

8. HPV is one of the most common sexually transmitted infections in the United States.

\section{Answer: TRUE}

TRUE FALSE

9. A pap smear can detect the HPV virus.

TRUE

FALSE

Answer: TRUE 
Appendix $\mathrm{H}$

\section{Decision-Making}

Directions: Please answer the following questions according to how you perceive the HPV vaccine.

1. What do you view as the greatest benefits of getting the HPV vaccination for yourself?

2. What do you view as the greatest drawbacks of getting the HPV vaccination for yourself?

Directions: If you have received the HPV vaccine, please answer the following question regarding what led you to get vaccinated and select the number that best describes your level of satisfaction with your decision.

What factors led you to get vaccinated?

How satisfied are you with your decision to receive the HPV vaccine?

Not at all Satisfied

12

3

4

5

Very Satisfied

$6 \quad 7$

Directions: If you have not received the HPV vaccine, please answer the following question regarding what keeps you from getting vaccinated and select the number that best describes your level of satisfaction with your decision.

What factors keep you from getting vaccinated?

How satisfied are you with your decision to not yet receive the HPV vaccine?

Not at all Satisfied

1

2

3

4

5

Very Satisfied

$6 \quad 7$


Appendix I

\section{HPV Vaccination Status}

Which of the following best describes your current situation?
a. Have completed the series of 3 shots for the HPV vaccine.
b. Have started, but not completed, the series of 3 shots for the HPV vaccine.
c. Have scheduled an appointment with my doctor to receive the HPV vaccine.
d. Have not received the HPV vaccine or scheduled an appointment to receive the HPV vaccine.

If you have received at least one of the HPV vaccine shots, please indicate the approximate date of vaccination:

$1^{\text {st }}$ shot
$2^{\text {nd }}$ shot
$3^{\text {rd }}$ shot

If you have received only one or two of the HPV vaccine shots, do you plan to complete the vaccine series?

\section{YES NO}


Appendix $\mathbf{J}$

HPV Vaccination Intentions

1. I want to get vaccinated against the human papillomavirus (HPV) within the next year. Strongly Disagree

12

3

4

5

Strongly Agree

2. I expect that I will get vaccinated against the human papillomavirus (HPV) within the next year.

Strongly Disagree

12

3

4

5

Strongly Agree

$6 \quad 7$

3. I do not intend to get vaccinated against the human papillomavirus (HPV) within the next year.

Strongly Disagree

12

3

4

5

Strongly Agree

4. I plan to make an appointment for an HPV vaccination within the next year.

Strongly Disagree

12

3

4

5

Strongly Agree

$6 \quad 7$ 


\section{Appendix K}

Dear Participant,

Thank you for your interest in this study, which is being conducted by researchers at the University of Maryland, College Park. Your participation will contribute important knowledge regarding people's attitudes toward a common sexually transmitted disease, HPV, and its prevention, the HPV vaccine. This questionnaire will take most people approximately 15-20 minutes to complete. It is important that you answer all questions in one sitting so, if you are completing this questionnaire on your own, please seek out a quiet place that is free from distractions while taking the study. At the end of the study, you will have the opportunity to seek additional information about the human papillomavirus (HPV).

In order to better understand the attitudes of people toward this health topic, it will be necessary for the researchers to ask questions about perceptions and behaviors of the participants that are relevant to the subject of sexually transmitted diseases (STDs). Some of these questions may be personal in nature, including items inquiring about sexual behaviors. The responses of participants who are sexually active, as well as those who are not currently active or never have been, are all vital to the success of this research. Due to the personal nature of some of this material, it is important for you to know that the information you give will be kept confidential. You will not be asked for your name and if you are submitting the last name of a professor to get class credit for taking the study, this information will be stored separately from the rest of your questionnaire and will be destroyed as soon as we report your participation. All information will be stored in a secure, locked location to which only the investigators have direct access.

Risks associated with this study may include feeling discomfort in response to some content or inadvertently disclosing your responses if the survey is not taken in private. However, you do not have to answer any questions that make you feel uncomfortable. Benefits include the likelihood that you will learn information about a common STD that might help to protect your health now or in the future and that you are contributing to research about an important health topic. Your participation in this study is voluntary and you may choose not to participate and may stop at any time. If you experience any difficulty in submitting your responses please contact the first researcher at the email address below.

If you have any questions or comments about the study, please feel free to contact either of the researchers (contact information below). If you have questions about your rights as a research subject, please contact the Institutional Review Board (also below). This research has been reviewed according to the University of Maryland, College Park IRB procedures for research involving human subjects. Thank you again for your participation. By giving your consent to participate, you indicate that: 1 . you are at least 18 years of age, 2 . the research has been explained to you, 3. your questions have been fully answered and 4. you freely and voluntarily choose to participate in this research project. If you agree with these statements and consent to participate, please click on the 'Continue' button below.

Kathryn Schaefer

Counseling Psychology Program

CAPS Department

University of Maryland

College Park, MD 20742
Mary Ann Hoffman, Ph.D. Professor, Counseling Psychology

CAPS Department

University of Maryland

College Park, MD 20742 


\section{Appendix L}

At the beginning of the study, we recommended that you take this survey alone in order to ensure that your responses were accurate and not influenced by others who may have been with you when you took the survey. You will receive class credit or be entered into a gift certificate drawing regardless of how you answer the following question, but we ask that you answer honestly.

Did you consult with anyone while taking this survey or did anyone else influence your answers?

\section{YES NO}

Thank you so much for participating in this study! Please read the following to make certain you get class credit, if applicable. This information will be kept separate from the rest of your responses.

If you are participating for class credit, please write the following below.

\section{Your university}

2. The last name of your instructor

3. A self-generated 4 digit ID number (ex. the last four digits of your cell phone, social security number, or student ID number).

In order to receive credit for participating in this study, please email your 4-digit number to the researcher at kschaefe@umd.edu.

If you are not taking this for class credit, please answer the questions below:

1. University name

2. Where you learned about this study (ex. "sorority" or "class 325 ").

If you are NOT taking this for class or community service credit and would like to enter a raffle for a $\$ 40$ gift certificate, please email the researcher (AFTER submitting your survey with the above information) at kschaefe@ umd.edu and put the word "raffle" as the subject line. 
Appendix M

\section{Links to More HPV Information}

1. This link leads to a fact sheet including Frequently Asked Questions about human papillomavirus by the Centers for Disease Control and Prevention:

http://www.cdc.gov/std/HPV/STDFact-HPV.htm

2. This link will connect you with lots of information about the HPV virus and its vaccine that has been compiled by the Centers Disease Control and Prevention:

http://www.cdc.gov/std/hpv/

3. This link to the American Social Health Association's (ASHA) National HPV and Cervical Cancer Prevention Resource Center has information, chat rooms, support groups, and publications about HPV, as well as ways to get involved in the prevention effort against HPV:

http://www.ashastd.org/hpv/hpv_overview.cfm

4. This link leads to information from the International Council on Infertility Information Dissemination on how the HPV vaccine could indirectly be helpful in preventing infertility:

http://www.inciid.org/article.php?cat=\&id=437 
Appendix N

Email to Participants

A research study about attitudes toward the HPV vaccine

Would you be interested in participating in a study exploring factors that affect a person's attitudes toward a common sexually transmitted disease (HPV) and its prevention (the HPV vaccine)? Participation requires completing a one-time online survey, which will take approximately 15-20 minutes to complete, on a secure website. In order to better understand the attitudes of people toward this health topic, it will be necessary for the researchers to ask questions about perceptions and behaviors of the participants that are relevant to the subject of sexually transmitted diseases (STDs). Some of these questions may be personal in nature, including items inquiring about sexual behaviors. The responses of people who are sexually active, as well as those who are not currently active or never have been, are all vital to the success of this research. Due to the personal nature of some of this material, it is important for you to know that the information you give will be kept confidential. Risks associated with this study may include feeling discomfort in response to some content or inadvertently disclosing your responses if the survey is not taken in private. However, you do not have to answer any questions that make you feel uncomfortable. Benefits of participation include the likelihood that you will learn information about a common STD that might help to protect your health now or in the future and that you are contributing to research about an important health topic. The study is being conducted by Ms. Kathryn Schaefer, a doctoral student, and Dr. Mary Ann Hoffman, Ph.D., a professor at the University of Maryland, College Park. 
Participation in this research is completely voluntary. If you are interested, visit the website below to be taken to PsychData, a secure website that hosts the survey. Once you are there you will be able to review a brief description of the survey, and read an informed consent form before you decide to participate.

Online survey: https://www.psychdata.com/s.asp?SID=132613 


\section{Appendix $\mathrm{O}$}

Scree Plot for Perceived Susceptibility

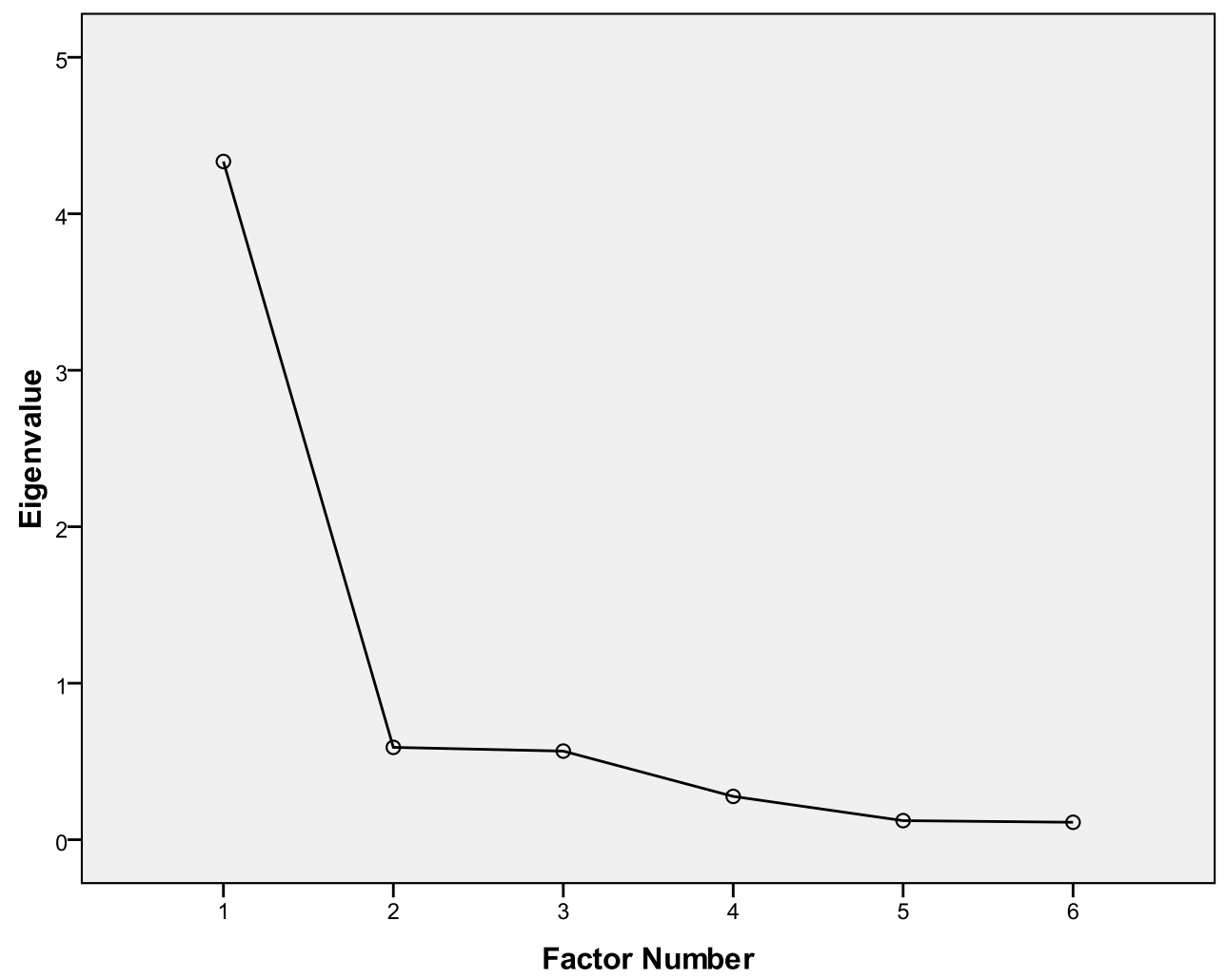

Total Variance Explained for Perceived Susceptibility

\begin{tabular}{|c|c|c|c|c|c|c|}
\hline \multirow[b]{2}{*}{ Factor } & \multicolumn{3}{|c|}{ Initial Eigenvalues } & \multicolumn{3}{|c|}{ Extraction Sums of Squared Loadings } \\
\hline & Total & $\%$ of Variance & Cumulative \% & Total & $\%$ of Variance & Cumulative \% \\
\hline 1 & 4.334 & 72.234 & 72.234 & 4.031 & 67.188 & 67.188 \\
\hline 2 & .590 & 9.834 & 82.068 & & & \\
\hline 3 & .566 & 9.436 & 91.503 & & & \\
\hline 4 & .277 & 4.612 & 96.115 & & & \\
\hline 5 & .122 & 2.030 & 98.145 & & & \\
\hline 6 & .111 & 1.855 & 100.000 & & & \\
\hline
\end{tabular}




\section{Appendix $\mathrm{P}$}

Scree Plot for Perceived Severity

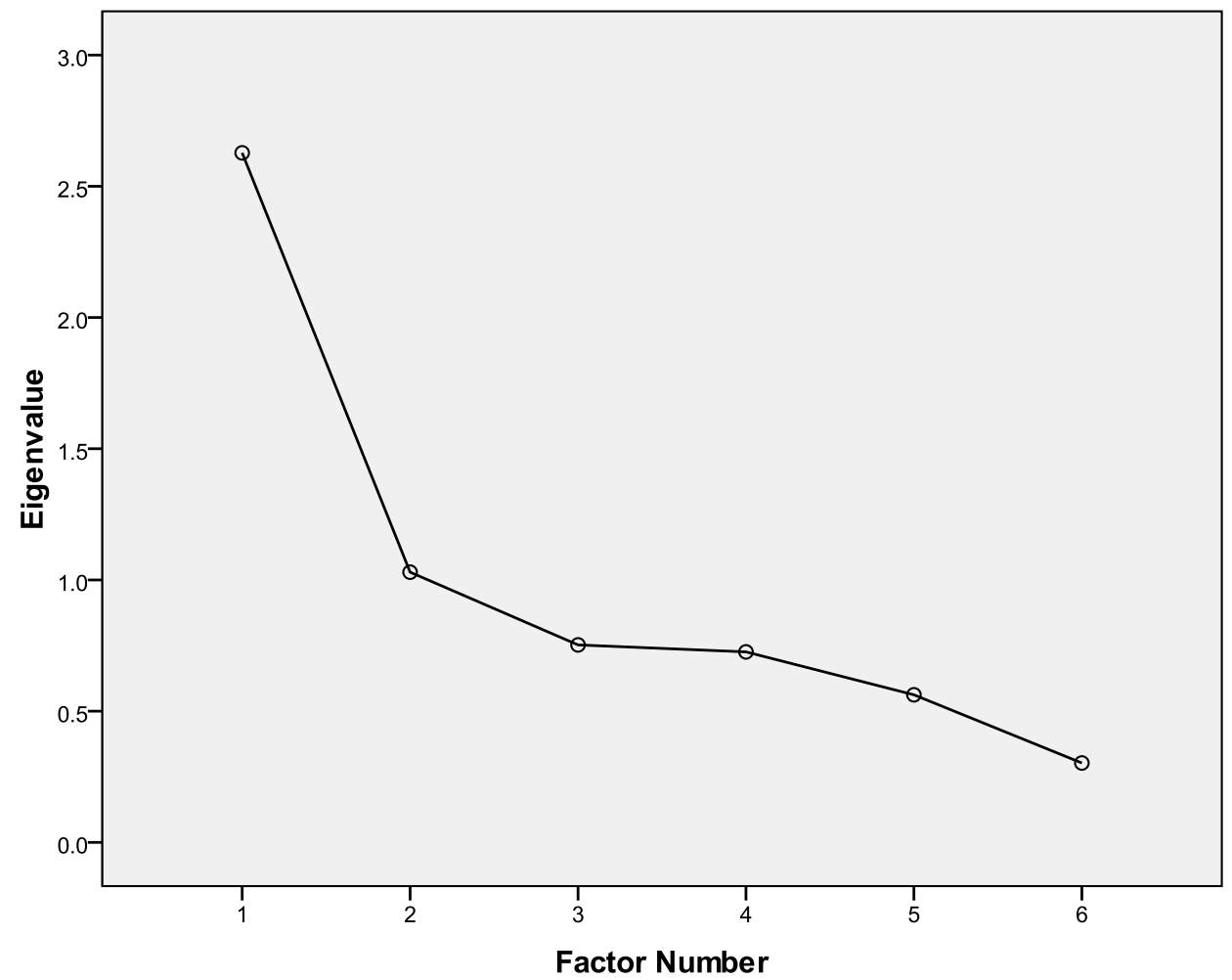

Total Variance Explained for Perceived Severity

\begin{tabular}{|c|c|c|c|c|c|c|}
\hline \multirow[b]{2}{*}{ Factor } & \multicolumn{3}{|c|}{ Initial Eigenvalues } & \multicolumn{3}{|c|}{ Extraction Sums of Squared Loadings } \\
\hline & Total & $\%$ of Variance & Cumulative \% & Total & $\%$ of Variance & Cumulative \% \\
\hline 1 & 2.627 & 43.788 & 43.788 & 2.079 & 34.642 & 34.642 \\
\hline 2 & 1.030 & 17.160 & 60.948 & & & \\
\hline 3 & .752 & 12.540 & 73.489 & & & \\
\hline 4 & .726 & 12.098 & 85.587 & & & \\
\hline 5 & .562 & 9.372 & 94.958 & & & \\
\hline 6 & .303 & 5.042 & 100.000 & & & \\
\hline
\end{tabular}




\section{Appendix Q}

Scree Plot for Perceived Benefits

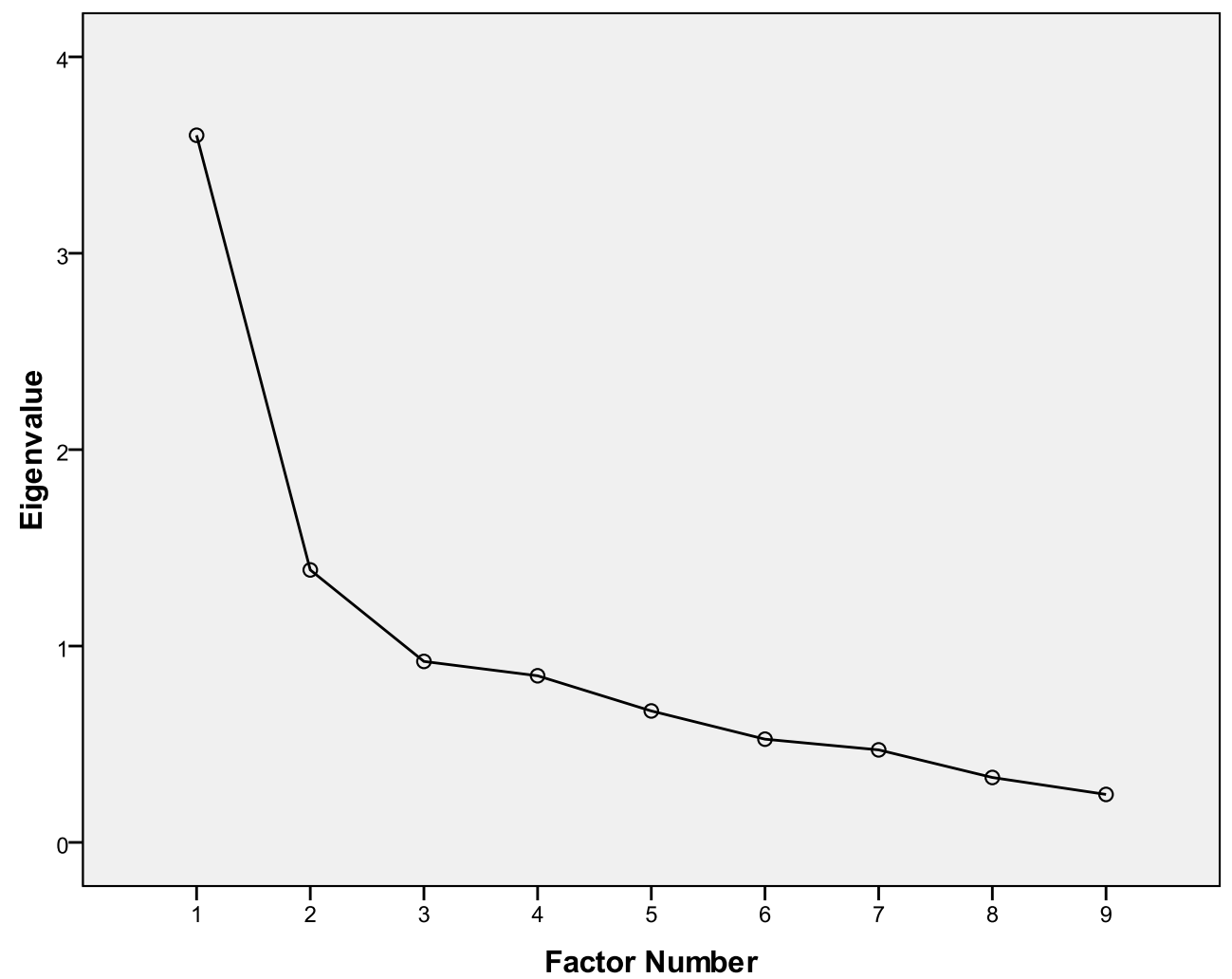

Total Variance Explained for Perceived Benefits

\begin{tabular}{|c|c|c|c|c|c|c|}
\hline \multirow[b]{2}{*}{ Factor } & \multicolumn{3}{|c|}{ Initial Eigenvalues } & \multicolumn{3}{|c|}{ Extraction Sums of Squared Loadings } \\
\hline & Total & $\%$ of Variance & Cumulative \% & Total & $\%$ of Variance & Cumulative \% \\
\hline 1 & 3.601 & 40.010 & 40.010 & 3.039 & 33.762 & 33.762 \\
\hline 2 & 1.388 & 15.417 & 55.427 & & & \\
\hline 3 & .922 & 10.241 & 65.667 & & & \\
\hline 4 & .849 & 9.429 & 75.096 & & & \\
\hline 5 & .669 & 7.438 & 82.534 & & & \\
\hline 6 & .526 & 5.841 & 88.376 & & & \\
\hline 7 & .471 & 5.236 & 93.611 & & & \\
\hline 8 & .331 & 3.674 & 97.285 & & & \\
\hline 9 & .244 & 2.715 & 100.000 & & & \\
\hline
\end{tabular}




\section{Appendix R}

Scree Plot for Perceived Barriers

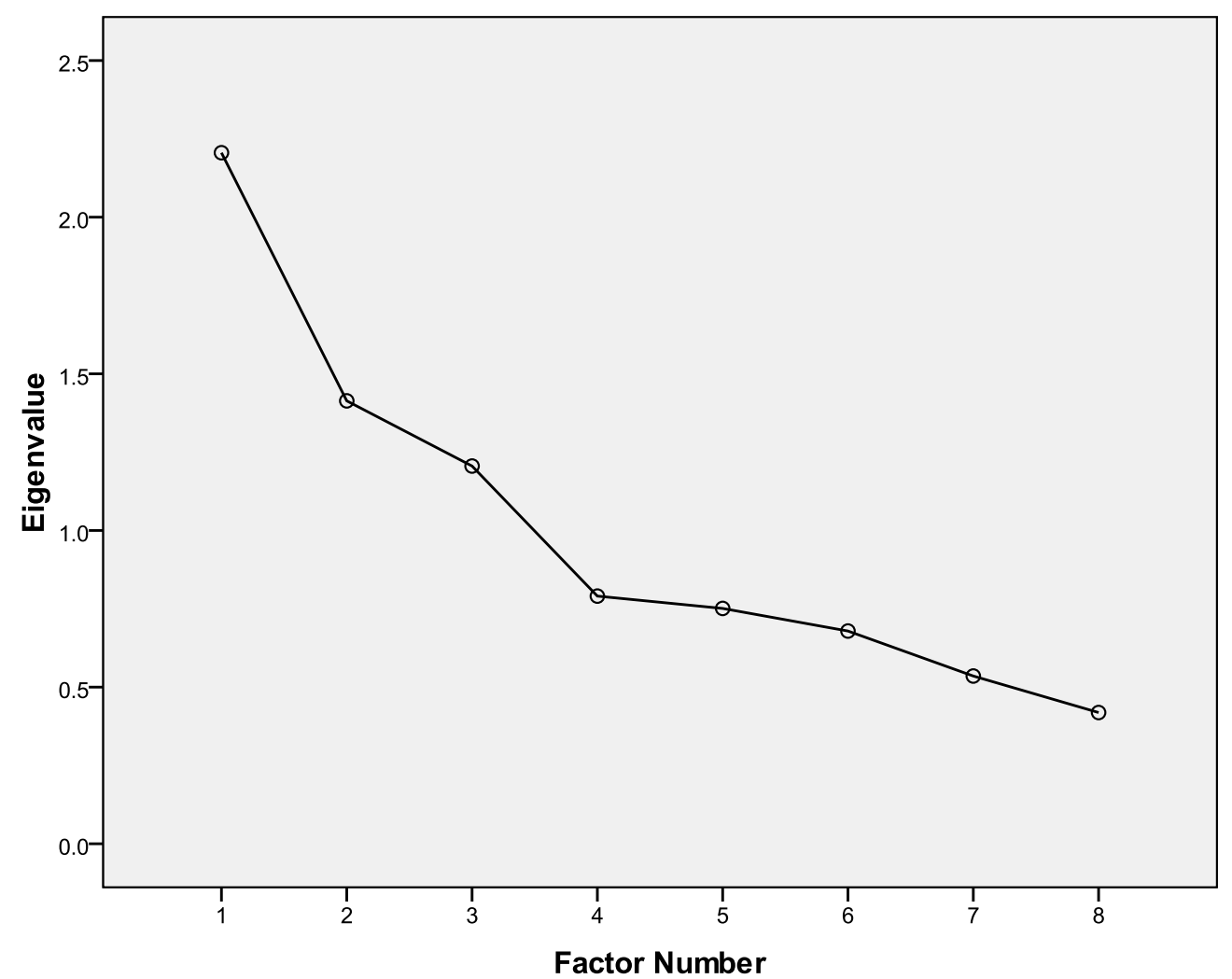

Total Variance Explained for Perceived Barriers

\begin{tabular}{|l|r|r|r|r|r|r|}
\hline \multirow{2}{*}{ Factor } & \multicolumn{4}{|c|}{ Initial Eigenvalues } & \multicolumn{2}{|c|}{ Extraction Sums of Squared Loadings } \\
\cline { 2 - 7 } & \multicolumn{1}{|c|}{ Total } & \% of Variance & Cumulative \% & Total & \% of Variance & Cumulative \% \\
\hline 1 & 2.206 & 27.571 & 27.571 & 1.574 & 19.680 & 19.680 \\
2 & 1.414 & 17.671 & 45.242 & & & \\
3 & 1.206 & 15.070 & 60.313 & & & \\
4 & .791 & 9.882 & 70.194 & & & \\
5 & .751 & 9.390 & 79.584 & & & \\
6 & .679 & 8.487 & 88.071 & & & \\
7 & .535 & 6.693 & 94.764 & & & \\
8 & .419 & 5.236 & 100.000 & & & \\
\hline
\end{tabular}




\section{References}

Abel, G. \& Brunton, C. (2005). Young people's use of condoms and their perceived vulnerability to sexually transmitted infections. Australian and New Zealand Journal of Public Health, 29, 254-260.

Abraham, I.L. \& Williams, B.M. (1991). Hypertensive elders perception and management of their disease: Health beliefs or health decisions? Journal of Applied Gerontology, 10, 444-454.

Adams, M., Jasani, B., \& Fiander, A. (2009). Prophylactic HPV vaccination for women over 18 years of age. Vaccine, 27, 3391-3394.

Aho, W.R. (1979). Participation of senior citizens in the Swine Flu inoculation programme: An analysis of health belief model variables in preventive health behavior, Journal of Gerontology, 34, 201-208.

Allen, J.D., Mohllajee, A.P., Shelton, R.C., Othus, M.K.D., Fontenot, H.B., \& Hanna, R. (2009). Stage of adoption of the human papillomavirus vaccine among college women. Preventive Medicine, 48, 420-425.

Ali, N.S. (2002). Prediction of coronary heart disease preventive behaviors in women: A test of the health belief model. Women \& Health, 35, 83-96.

American Society for Colposcopy and Cervical Pathology (2003). What women should know about HPV and cervical health. Retrieved on May 5, 2006 from http://www.asccp.org/patient_edu.shtml

Armitage, C.J., \& Conner, M. (2000). Social cognition models and health behavior: A structured review. Psychology and Health, 15, 173-189. 
Baer, H., Allen, S., \& Braun, L. (2000). Knowledge of human papillomavirus infection among young men and women: Implications for health education and research. Journal of Community Health, 25, 67-78.

Bandura, A. (1977). Self-efficacy: Toward a unifying theory of behavior change. Psychological Review, 84, 191-215.

Bandura, A. (1986). Social foundations of thought and action. Englewood Cliffs, NJ: Prentice-Hall.

Baron, R. M., \& Kenny, D. A. (1986). The moderator-mediator variable distinction in social psychological research: Conceptual, strategic, and statistical considerations. Journal of Personality and Social Psychology, 51, 1173-1182.

Barton, S.E., Maddox, P.H., Jenkins, D., Edwards, R., Cuzick, J., \& Singer, A. (1988). Effect of cigarette smoking on cervical epithelial immunity: a mechanism for neoplastic change? Lancet, 17, 652-654.

Bauer, H.M., Ting, Y., Greer, C.E., Chambers, J.C., Tashiro, C.J., \& Chimera, J., et al. (1991). Genital human papillomavirus infection in female university students as determined by a PCR-based method. The Journal of the American Medical Association, 265, 472-477.

Becker, M.H., Maiman, L.A., Kirscht, J.P., Haefner, D.P., \& Drachman, R.H. (1977). The health belief model and prediction of dietary compliance: A field experiment. Journal of Health and Social Behavior, 18, 348-366.

Becker, T.M., Stone, K.M., \& Alexander, E.R. (1987). Genital human papillomavirus infection: A growing concern. Obstetrics and Gynecology Clinics of North America, 14, 389-396. 
Boehner, C.W., Howe, S.R., Bernstein, D.I., \& Rosenthal, S.L. (2003). Viral sexually transmitted disease vaccine acceptability among college students. Sexually Transmitted Diseases, 30, 774-778.

Bosch, F.X., \& de Sanjose, S. (2003). Chapter 1: Human papillomavirus and cervical cancer-burden and assessment of causality. Journal of the National Cancer Institute Monographs, 31, 3-13.

Brewer, N.T., Chapman, G.B., Gibbons, F.X., Gerrard, M., McCaul, K.D., \& Weinstein, N.D. (2007). Meta-analysis of the relationship between risk perception and health behavior: The example of vaccination, Health Psychology, 26, 136-145.

Brewer, N.T. \& Fazekas, K.I. (2007). Predictors of HPV vaccine acceptability: A theoryinformed, systematic review, Preventive Medicine, 45, 107-114.

Buchanan, J.A. (2008). Comparing the health belief model and theory of planned behavior in predicting intent to vaccinate against the human papillomavirus in college women (Doctoral dissertation, Indiana State University, 2008).

Dissertation Abstracts International, 69, 4411B.

Burak, L.J., \& Meyer, M. (1997). Using the health belief model to examine and predict college women's cervical cancer screening beliefs and behavior. Health Care for Women International, 18, 251-262.

Burk, R.D., Ho, G.Y., Beardsley, L., Lempa, M., Peters, M., \& Bierman, R. (1996). Sexual behavior and partner characteristics are the predominant risk factors for genital human papillomavirus infection in young women. Journal of Infectious Diseases, 174, 679-689. 
Burkett, B.J., Peterson, C.M., Birch, L.M., Brennan, C., Nuckols, M.L., \& Ward, B.E., et al. (1992). The relationship between contraceptives, sexual practices, and cervical human papillomavirus infection among a college population. Journal of Clinical Epidemiology, 45, 1295-1302.

Castellsague X. Bosch, F.X., Munoz, N., Meijer, C.J., Shah, K.V., \& De Sanjose, S., et al. (2002). Male circumcision, penile human papillomavirus infection, and cervical cancer in female partners. The New England Journal of Medicine, 346, 1105-1112.

Cates, W. (1999). Estimated of the incidence and prevalence of sexually transmitted diseases in the United States. Sexually Transmitted Diseases, 26, 2-7.

Cattell, R. B. (1966). The scree test for the number of factors. Multivariate Behavioral Research, 1, 245-276.

Center for Disease Control and Prevention. (2009). National, state, and local area vaccination coverage among adolescents aged 13-17 years - United States, 2009. Morbidity and Mortality Weekly Report, 58, 997-1001.

Center for Disease Control and Prevention. (2008). Genital HPV infection: CDC fact sheet. Retrieved on November 5, 2008, from http://www.cdc.gov/std/HPV/STDFact-HPV.htm.

Cervical Cancer. (1996, April). NIH Consensus Statement, 14, 1-38.

Champion, V.L. (1984). Instrument development for the health belief model constructs. Advances in Nursing Science, 6, 73-85. 
Chan, S.S.C, Cheung, T.H., Lo, W.K., \& Chung, T.K.H. (2007). Women's attitudes on human papillomavirus vaccination to their daughters. Journal of Adolescent Health, 41, 204-207.

Chapman, G.B. \& Coups, E.J. (1999). Predictors of influenza vaccine acceptance among healthy adults. Preventive Medicine, 29, 249-262.

Chaturvedi, A.K., Engels, E.A., Anderson, W.F., \& Gillison, M.L. (2008). Incidence trends for human papillomavirus-related and -unrelated oral squamous cell carcinomas in the United States. Journal of Clinical Oncology, 26, 612-619.

Chesson, H.W., Blandford, J.M., Gift, T.L., Tao, G., \& Irwin, K.L. (2004). The estimated direct medical cost of sexually transmitted diseases among American youth, 2000. Perspectives on Sexual and Reproductive Health, 36, 11-19.

Chuang, T.Y., Perry, H.O., Kurland, L.T., \& Ilstrup, D.M. (1984). Condyloma acuminatum in Rochester, Minn., 1950-1978. I. Epidemiology and clinical features. Archives of Dermatology, 120, 469-475.

Clifford, G.M., Smith, J.S., Plummer, M., Munoz, N., \& Franceschi, S. (2003). Human papillomavirus types in invasive cervical cancer worldwide: A meta-analysis. British Journal of Cancer, 88, 63-73.

Cohen, J. (1988). Statistical Power Analysis for the Behavioral Sciences ( $2^{\text {nd }}$ ed.). Hillsdale NJ: Erlbaum.

Conaglen, H.M., Hughes, R., Conaglen, J.V., \& Morgan, J. (2001). A prospective study of the psychological impact on patients of first diagnosis of human papillomavirus. International Journal of STD \& AIDS, 12, 651-658. 
Constantine, N.A., \& Jerman, P. (2007). Acceptance of human papillomavirus vaccination among Californian parents of daughters: A representative statewide analysis. Journal of Adolescent Health, 40, 108-115.

Costar, H. (2008). Creating persuasive health messages: Consideration of future consequences and intention to pursue vaccination against human papillomavirus (Doctoral dissertation, University of Maryland, College Park, 2006). Dissertation Abstracts International, 69, 1372B.

Cummings, K.M., Jette, A.M., \& Brock, B.M. (1979). Psychological determinants of immunization behaviour in a Swine Influenza campaign, Medical Care, 17, 639649.

Davis, K., Dickman, E.D., Ferris, D., \& Dias, J.K. (2004). Human papillomavirus vaccine acceptability among parents of 10- to 15-year-old adolescents. Journal of Lower Genital Tract Disease, 8, 188-194.

Dempsey, A.F., Zimet, G.D., Davis, R.L., \& Koutsky, L. (2006). Factors that are associated with parental acceptance of human papillomavirus vaccines: A randomized intervention study of written information about HPV. Pediatrics, 117, 1486-1493.

Dinh, T., Sternberg, M., Dunne, E.F., \& Markowitz, L.E. (2008). Genital Warts Among 18- to 59-Year-Olds in the United States, National Health and Nutrition Examination Survey, 1999-2004. Sexually Transmitted Diseases, 35, 357-360.

Elbasha, E.H., Dasbach, E.J., \& Insinga, R.P. (2007). Model for assessing human papillomavirus vaccination strategies. Emerging Infectious Diseases, 13, 28-41. 
Ethier, K. A., Kershaw, T., Niccolai, L., Lewis, J.B., \& Ickovics, J.R. (2003). Adolescent women underestimate their susceptibility to sexually transmitted diseases. Sexually Transmitted Infections, 79, 408-411.

Fabrigar, L. R., Wegener, D. T., MacCallum, R. C., \& Strahan, E. J. (1999). Evaluating the use of exploratory factor analysis in psychological research. Psychological Methods, 4, 272-299.

Faul, F., Erdfelder, E., Lang, A.G., \& Buchner, A. (2007). G*Power 3: A flexible statistical power analysis program for the social, behavioral, and biomedical sciences. Behavior Research Methods, 39, 175-191.

Filiberti, A., Tamburini, M., Stefanon, B., Merola, M., Bandieramonte, G., \& Ventafridda, V., et al. (1993). Psychological aspects of genital human papillomavirus infection: A preliminary report. Journal of Psychosomatic Obstetrics \& Gynecology, 14, 145-152.

Fishbein, M. \& Ajzen, I. (1975). Belief, attitude, intention, and behavior: an introduction to theory and research. Addison Wesley, Reading Mass.

Fletcher, P.C. \& Bryden, P.J. (2005). Preliminary examination of cervical health practices and knowledge among university-aged females. College Student Journal, 39, 469477.

Fleurence, R.L., Dixon, J.M., Milanova, T.F., \& Beusterien, K.M. (2007). Review of the economic and quality-of-life burden of cervical human papillomavirus disease. American Journal of Obstetrics and Gynecology, 196, 206-212.

Ford, C.A., Jaccard, J., Millstein, S.G., Bardsley, P.E., \& Miller, W.C. (2006). Perceived risk of chlamydial and gonococcal infection among sexually experienced young 
adults in the United States. Perspectives on Sexual and Reproductive Health, 36, 258-264.

Frazier, P.A., Tix, A.P, \& Barron, K.E. (2004). Testing moderator and mediator effects in counseling psychology research. Journal of Counseling Psychology, 51, 115-134.

Friedman, A.L., \& Shepeard, H. (2007). Exploring the knowledge, attitudes, beliefs, and communication preferences of the general public regarding HPV: Findings from CDC focus group research and implications for practice. Health Education \& Behavior, 34, 471-485.

Frisch, M., Glimelius, B., Van Den Brule, A.J.C., Wohlfahrt, J., Meijer, C.J.L., \& Walboomers, J.M.M., et al. Sexually transmitted infection as a cause of anal cancer. The New England Journal of Medicine, 337, 1350-1358.

FUTURE II Study Group (2007). Quadrivalent vaccine against human papillomavirus to prevent high-grade cervical lesions. The New England Journal of Medicine, 356, 1915-1927.

Gardasil. (2009). Important information about Gardasil. Retrieved on November 5, 2008, from http://www.gardasil.com/.

Garland, S.M., Hernandez-Avila, M., Wheeler, C.M., Perez, G., Harper, D.M., \& Leodolter, S. (2007). Quadrivalent vaccine against human papillomavirus to prevent anogenital diseases. The New England Journal of Medicine, 356, 19281943.

Gerend, M.A., \& Magloire, Z.F. (2008). Awareness, knowledge, and beliefs about human papillomavirus in a racially diverse sample of young adults. Journal of Adolescent Health, 42, 237-242. 
Gerend, M.A., \& Barley, J. (2008). Human papillomavirus vaccine acceptability among young adult men. Sexually Transmitted Diseases, 36, 58-62.

Gerend, M.A., Lee, S.C., \& Shepherd, J.E. (2006). Predictors of human papillomavirus vaccination acceptability among underserved women. Sexually Transmitted Diseases, 34, 468-471.

Gerend, M.A., \& Shepherd, J.E. (2007). Using message framing to promote acceptance of the human papillomavirus vaccine. Health Psychology, 26, 745-752.

Gerend, M.A., Shepherd, J.E., \& Monday, K.A. (2007). Behavioral frequency moderates the effects of message framing on HPV vaccine acceptability. Annals of Behavioral Medicine, 35, 221-229.

Gillison, M.L., Chaturvedi, A.K., \& Lowy, D.R. (2008). HPV prophylactic vaccines and the potential prevention of noncervical cancers in both men and women. Cancer, 113, 3036-3046.

Gissmann, L., Wolnikt, L., Ikenberg, H., Koldovskyt, U., Schnurcht, H.G., \& Hausen, H.Z. (1983). Human papillomavirus types 6 and 11 DNA sequences in genital and laryngeal papillomas and in some cervical cancers. Proceedings of the National Academy of Sciences, 80, 560-563.

Gorsuch, R.L. (1983). Factor analysis (2nd ed.). Hillsdale, NJ: Lawrence Earlbaum Associates.

Grant, D., Kravitz-Wirtz, N., Breen, N., Tiro, J.A., \& Tsui, J. (2009, Februrary). One in four california adolescent girls have had human papillomavirus vaccination. UCLA Health Policy Research Brief, 1-6. 
Graziottin, A. \& Serafini, A. (2009). HPV infection in women: Psychosexual impact of genital warts and intraepithelial lesions. The Journal of Sexual Medicine, 6, 633645.

Harrison, J.A., Mullen, P.D., \& Green, L.W. (1992). A meta-analysis of studies of the health belief model with adults, Health Education Research, 7, 107-116.

Ho, G.Y.F., Bierman, R., Beardsley, L., Chang, C.J., \& Burk, R.D. (1998). National history of cervicovaginal papillomavirus infection in young women. The New England Journal of Medicine, 338, 423-428.

Hoffman, M. (2002). Unpublished manuscript.

Houppart, K. (2007, March 8). Who's afraid of Gardasil? Retrieved on June 21, 2009

from www.thenation.com/doc/20070326/houppert

HPV Vaccine - Questions and Answers for the Public. (July, 17, 2008). Center for Disease Control. Retrieved on June 21, 2009 from http://www.cdc.gov/vaccines/vpd-vac/hpv/hpv-vacsafe-effic.htm.

Insinga, R.P., Dasbach, E.J., \& Myers, E.R. (2003). The health and economic burden of genital warts in a set of private health plans in the United States. Clinical Infectious Diseases, 36, 1397-1403.

Ireland, J.A., Reid, M., Powell, R., \& Petrie, K.J. (2005). The role of illness perceptions: Psychological distress and treatment-seeking delay in patients with genital warts. International Journal of STD and AIDS, 16, 667-670.

Janz, N. \& Becker, M.H. (1984). The health belief model: A decade later, Health Education Quarterly, 11, 1-47. 
Jones, M., \& Cook, R. (2008). Intent to Receive an HPV Vaccine Among University Men and Women and Implications for Vaccine Administration. Journal of American College Health, 57, 23-31.

Kahn, J. H. (2006). Factor analysis in counseling psychology research, training, and practice: Principles, advances, and applications. The Counseling Psychologist, 34, 684-718.

Kahn, J.A., Rosenthal, S.L., Hamann, T., \& Bernstein, D.I. (2003). Attitudes about human papillomavirus vaccine in young women. International Journal of STD \& AIDS, 14, 300-306.

Kahn, J.A., Rosenthal, S.L., Jin, Y., Huang, B., Namakydoust, A., \& Zimet, G.D. (2008). Rates of human papillomavirus vaccination, attitudes about vaccination, and human papillomavirus prevalence in young women. Obstetrics \& Gynecology, 111, 1103-1110.

Kataja, V., Syrjänen, S., Yliskoski, M., Hippelïnen, M., Väyrynen, M., Saarikoski, S., et al. (1993). Risk factors associated with cervical human papillomavirus infections: A case-control study. American Journal of Epidemiology, 138, 735-745.

King, A.N. (2006). Factors influencing the decision to be tested for HIV among heterosexual college students (Doctoral dissertation, Southern Illinois University, 2006) Dissertation Abstracts International, 67, 2209B.

Koutsky, L. (1997) Epidemiology of Genital Human Papillomavirus Infection. The American Journal of Medicine, 102, 3-8. 
Lambert, E.C. (2001). College students' knowledge of human papillomavirus and effectiveness of a brief educational intervention. Journal of the American Board of Family Practice, 14, 178-183.

Linnehan, M.J.E. \& Groce, N.E. (1999). Psychosocial and educational services for female college students with genital human papillomavirus infection. Family Planning Perspectives, 31, 137-141.

MacCallum, R. C., Widaman, K. F., Zhang, S., \& Hong, S. (1999). Sample size in factor analysis. Psychological Methods, 4, 84-89.

Maggino, T., Casadei, D., Panontin, E., Fadda, E., Zampieri, M.C., \& Donà, M.A., et al. (2007). Impact of an HPV diagnosis on the quality of life in young women. Gynecologic Oncology, 107, 175-179.

Maimen, L.A., Becker, M.H., Kirscht, J.P., Haefner, D.P., \& Drachman, R.H. (1977). Sclaes for measuring health belief model dimensions: A test of predictive value, internal consistency and relationships among beliefs. Health Education Quarterly, 4, 215-231.

Maw, R.D., Reitano, M., \& Roy, M. (1998). An international survey of patients with genital warts: Perceptions regarding treatment and impact on lifestyle. International Journal of STD \& AIDS, 9, 571-578.

Mayo Clinic (Feb 2009). Genital Warts. http://www.mayoclinic.com/health/genitalwarts/DS00087/DSECTION=causes. Retrieved on June 17, 2009.

McCaffery, K., Waller, J., Forrest, S., Cadman, L., Szarewski, A., \& Wardlec J. (2004). Testing positive for human papillomavirus in routine cervical screening: 
Examination of psychosocial impact. British Journal of Obstetrics and Gynecology, 111, 1437-1443.

Melbye, M., Rabkin, C., Frisch, M., \& Biggar, R.J. (1994). Changing patterns of anal cancer incidence in the United States, 1940-1989. American Journal of Epidemiology, 139, 772-780.

Mork, J., Lie, K., Glattre, E., Hallmans, G., Jellum, E., \& Koskela, P., et al. (2001). Human papillomavirus infection as a risk factor for squamous-cell carcinoma of the head and neck. The New England Journal of Medicine, 344, 1125-1131.

Moscicki, A.B., Hills, N., Shiboski, S., Powell, K., Jay, N., \& Hanson, E., et al. (2001). Risks for incident human papillomavirus infection and low-grade squamous intraepithelial lesion development in young females. Journal of American Medical Association, 285, 2995-3002.

Munoz, N., Bosch, F.X., de Sanjose, S., Herrero, R., Castellsague, X., Shah, K.V., et al. (2003). Epidemiologic classification of human papillomavirus types associated with cervical cancer. The New England Journal of Medicine, 348, 518-527.

Muñoz-Silva, A., Sánchez-García, M., Nunes, C., \& Martins, A. (2007). Gender differences in condom use prediction with Theory of Reasoned Action and Planned Behaviour: The role of self-efficacy and control. AIDS Care: Psychological and Socio-medical Aspects of AIDS/HIV, 19, 1177-1181. National Cancer Institute, (2008). Human papillomavirus and cancer: questions and answers. Retrieved on January, 7, 2009 from http://www.cancer.gov/cancertopics/ factsheet/Risk/HPV. 
National Conference of State Legislatures. (June 2009). HPV Vaccine. Retrieved on June $22^{\text {nd }}$ from http://www.ncsl.org/IssuesResearch/Health/HPVVaccineState Legislation/tabid/ 14381/Default.aspx.

Orbell, S., Crombie, I., \& Johnston, G. (1996). Social cognition and social structure in the prediction of cervical screening uptake. British Journal of Health Psychology, 1, $35-50$.

Pascual, A., Pariente, M., Godinez, J.M., Sanchez-Prieto, R., Atienzar, M, Segura, M., et al. (2007). High prevalence of human papillomavirus 16 in penile carcinoma. Histology and Histopathology, 22, 177-183.

Petäjä, T., Keränen, H., Karppa, T., Kawa, A., Lantela, S., Siitari-Mattila, M., et al. (2009). Immunogenicity and safety of human papillomavirus (HPV)-16/18 AS04adjuvanted vaccine in healthy boys aged 10-18 years. Journal of Adolescent Health, 44, 33-40.

Pichichero, M.E. (2007). Who should get the HPV vaccine? The Journal of Family Practice, 56, 197-202.

Prochaska, J.O. \& DiClemente, C.C. (1984). The Transtheoretical Approach: Crossing Traditional Boundaries of Change. Dow Jones/Irwin, Homewood, IL.

Ramirez, J.E., Ramos, D.M., Clayton, L., Kanowitz, S., \& Moscicki, A.B. (1997). Genital human papillomavirus infections: Knowledge, perception of risk, and actual risk in a nonclinic population of young women. Journal of Women's Health, 6, 113121. 
Reinisch, J.M., Sanders, S.A., Hill, C.A., \& Ziemba-Davis, M. (1992). High-risk sexual behavior among heterosexual undergraduates at a midwestern university. Family Planning Perspectives, 24, 116-121.

Reports of Health Concerns Following HPV Vaccination. (June 10, 2009). Center for Disease Contrl. Retrieved on June 21, 2009 from http://www.cdc.gov/vaccinesafety/ vaers/gardasil.htm.

Romano, J.L., \& Netland, J.D. (2008). The application of the theory of reasoned action and planned behavior to prevention science in counseling psychology. The Counseling Psychologist, 36, 777-806.

Ronis, D.L. (1992). Conditional health threats: Health beliefs, decisions, and behaviors among adults. Health Psychology, 11, 127-134.

Ronis, D.L. \& Harel, Y. (1989). Health beliefs and breast examination behaviours: Analysis of linear structural relations, Journal of Psychology and Health, 3, 259285.

Rosenstock, , I.M. (1991). The health belief model: Explaining health behaviors through expectancies. In K. Glanz, F.M. Lewis, \& B. Rimer (Eds.), Health behavior and health education (39-62). San Francisco: Jossey-Bass Health Series.

Rosenstock, I.M., Strecher, V.J., \& Becker, M.H. (1988). Social learning theory and the health belief model. Health Education Quarterly, 15, 175-183.

Sellors, J.W., Karwalajtys, T.L., Kaczorowski, J, Mahony, J.B., Lytwyn, A., Chong, S., et al. (2003). Incidence, clearance and predictors of human papillomavirus infection in women. Canadian Medical Association Journal, 168, 421-425. 
Sheeran, P. \&Abraham, C. (1996). The health belief model. In M. Conner \& P. Norman (Eds.), Predicting Health Behavior: Research and Practice with Social Cognition Models (23-61). Buckingham: Open University Press.

Smith, J.S., Backes, D.M., Hoots, B.E., Kurman, R.J., \& Pimenta, J.M. (2009). Human papillomavirus type-distribution in vulvar and vaginal cancers and their associated precursors. Obstetrics \& Gynecology, 113, 917-924.

Souza, G.D., Kreimer, A.R., Viscidi, R., Pawlita, M., Fakhry, C., \& Koch, W.M. (2007). Case-control study of human papillomavirus and oropharyngeal cancer. The New England Journal of Medicine, 356, 1944-1956.

Strecher, V.J., Champion, V.L., \& Rosenstock, I.M. (1997). The health belief model and health behavior. In D.S. Gochman (Ed.), Handbook of Health Behavior Research I: Personal and Social Determinants (71-91). New York: Plenum Press.

Strecher, V.J., DeVellis, B.M., Becker, M.H., \& Rosenstock, I.M. (1986). The role of self-efficacy in achieving health behavior change. Health Education Quarterly, $13,73-92$.

Strecher, V.J. \& Rosenstock, I.M. (1997). The health belief model. In K. Glanz, F.M. Lewis, \& B. Rimer (Eds.), Health behavior and health education (2 ${ }^{\text {nd }}$ ed., 41-57). San Francisco: Jossey-Bass Health Series.

Thomas, K.K., Hughes, J.P., Kuypers, J.M., Kiviat, N.B., Lee, S.K., \& Adam, D.E., et al. (2001). Concurrent and sequential acquisition of different genital human papillomavirus types. Journal of Infectious Diseases, 182, 1097-1102.

Tinsley, H.E. \& Tinsley, D.J. (1987). Uses of factor analysis in counseling psychology research. Journal of Counseling Psychology, 34, 414-424. 
Trottier, H. \& Franco, E.L. (2006). The epidemiology of genital human papillomavirus infection. Vaccine, 24, 4-15.

U.S. Cancer Statistics Working Group. United States Cancer Statistics: 1999-2005 Incidence and Mortality Web-based Report. Atlanta (GA): Department of Health and Human Services, Centers for Disease Control and Prevention, and National Cancer Institute; 2009. Available at: http://www.cdc.gov/uscs.

Vail-Smith, K. \& White, D.M. (1992). Risk level, knowledge, and preventive behavior for human papillomaviruses among sexually active college women. Journal of American College Health, 40, 227-230.

Villa, L.L., Costa, R.L.R., Petta, C.A., Andrade, R.P., Ault, K.A., Giuliano, A.R., et al. (2005). Prophylactic quadrivalent human papillomavirus (types 6, 11, 16, and 18) L1 virus-like particle vaccine in young women: a randomised double-blind placebo-controlled multicentre phase II efficacy trial. Lancet Oncology, 6, 271-278.

Walboomers, J.M.M, Jacobs, M.V., Manos, M.M., Bosch, F.X., Kummer, J.A., Shah, K.V., et al. (1999). Human papillomavirus is a necessary cause of invasive cervical cancer worldwide. The Journal of Pathology, 189, 12-19.

Weinstock, H., Berman, S., \& Cates, W. (2004). Sexually transmitted diseases among american youth: Incidence and prevalence estimates, 2000. Perspectives on Sexual and Reproductive Health, 36, 6-10.

Winer, R.L., Hughes, J.P., Feng, Q., O’Reilly, S., Kiviat, N.B., Holmes, K.K., et al. (2006). Condom use and the risk of genital human papillomavirus infection in young women. The New England Journal of Medicine, 354, 2645-2654. 
Winer, R.L., Lee, S.K., Hughes, J.P., Adam, D.E., Kiviat, N.B., \& Koutsky, L.A. (2003). Genital human papillomavirus infection: Incidence and risk factors in a cohort of female university students. American Journal of Epidemiology, 157, 218-226.

Yacobi, E., Tennant, C. Ferrante, J., Pal, N., \& Roetzheim, R. (1999). University students' knowledge and awareness of HPV. Preventive Medicine, 28, 535-541.

Zimet, G.D., Mays, R.M., Winston, Y., Kee, R., Dickes, J., \& Su, L., 2000. Acceptability of human papillomavirus immunization. Journal of Women's Health and GenderBased Medicine, 9, 47-50. 